\title{
Statistical mechanics of two-dimensional vortices and stellar systems
}

\author{
Pierre-Henri Chavanis \\ Laboratoire de Physique Quantique, \\ Université Paul Sabatier, \\ 118, route de Narbonne \\ 31062 Toulouse, France
}

\begin{abstract}
The formation of large-scale vortices is an intriguing phenomenon in twodimensional turbulence. Such organization is observed in large-scale oceanic or atmospheric flows, and can be reproduced in laboratory experiments and numerical simulations. A general explanation of this organization was first proposed by Onsager (1949) by considering the statistical mechanics for a set of point vortices in two-dimensional hydrodynamics. Similarly, the structure and the organization of stellar systems (globular clusters, elliptical galaxies,...) in astrophysics can be understood by developing a statistical mechanics for a system of particles in gravitational interaction as initiated by Chandrasekhar (1942). These statistical mechanics turn out to be relatively similar and present the same difficulties due to the unshielded long-range nature of the interaction. This analogy concerns not only the equilibrium states, i.e. the formation of large-scale structures, but also the relaxation towards equilibrium and the statistics of fluctuations. We will discuss these analogies in detail and also point out the specificities of each system.
\end{abstract}

\section{Introduction}

Two-dimensional flows with high Reynolds numbers have the striking property of organizing spontaneously into coherent structures (the vortices) which dominate the dynamics [93] (see Fig. 1). The robustness of Jupiter's Great Red Spot, a huge vortex persisting for more than three centuries in a turbulent shear between two zonal jets, is probably related to this general property. Some other coherent structures like dipoles (pairs of cyclone/anticyclone) and sometimes tripoles have been found in atmospheric or oceanic systems and can persist during several days or weeks responsible for atmospheric blocking. Some astrophysicists invoke the existence of organized vortices in the gaseous component of disk galaxies in relation with the emission of spiral density waves [99]. It has also been proposed that planetary formation might have begun inside persistent gaseous vortices born out of the protoplanetary nebula [5, 121, 15, 60, 33 (see Fig. 2). As a result, hydrodynamical vortices occur in a wide variety of geophysical or astrophysical situations and their robustness demands a general understanding.

Similarly, it is striking to observe that self-gravitating systems follow a kind of organization despite the diversity of their initial conditions and their environement [9] (see Fig. 3). This organization is illustrated by morphological classification schemes such as the Hubble sequence for galaxies and by simple 
rules which govern the structure of individual self-gravitating systems. For example, elliptical galaxies display a quasi-universal luminosity profile described by de Vaucouleur's $R^{1 / 4}$ law and most of globular clusters are well-fitted by the Michie-King model. On the other hand, the flat rotation curves of spiral galaxies can be explained by the presence of a dark matter halo with a density profile decreasing as $r^{-2}$ at large distances. The fractal nature of the interstellar medium and the large scale structures of the universe also display some form of organization.
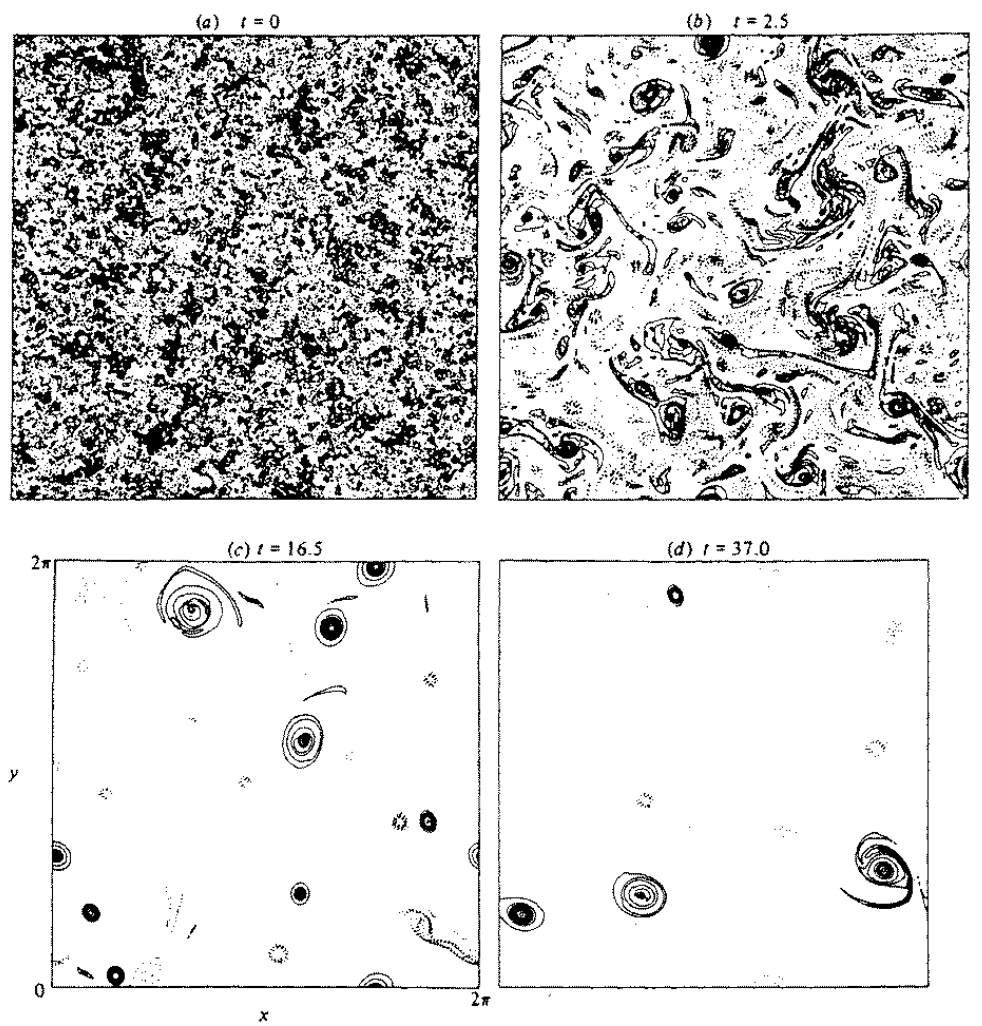

Fig. 1. Self-organization of two-dimensional turbulent flows into large-scale vortices 93. These vortices are long-lived and dominate the dynamics.

The question that naturally emerges is what determines the particular configuration to which a self-gravitating system or a large-scale vortex settles. It is possible that their actual configuration crucially depends on the conditions that prevail at their birth and on the details of their evolution. However, in view of their apparent regularity, it is tempting to investigate whether their organization can be favoured by some fundamental physical principles like those of thermodynamics and statistical physics. We ask therefore if the actual states of self-gravitating systems in the universe and coherent vortices in two-dimensional 

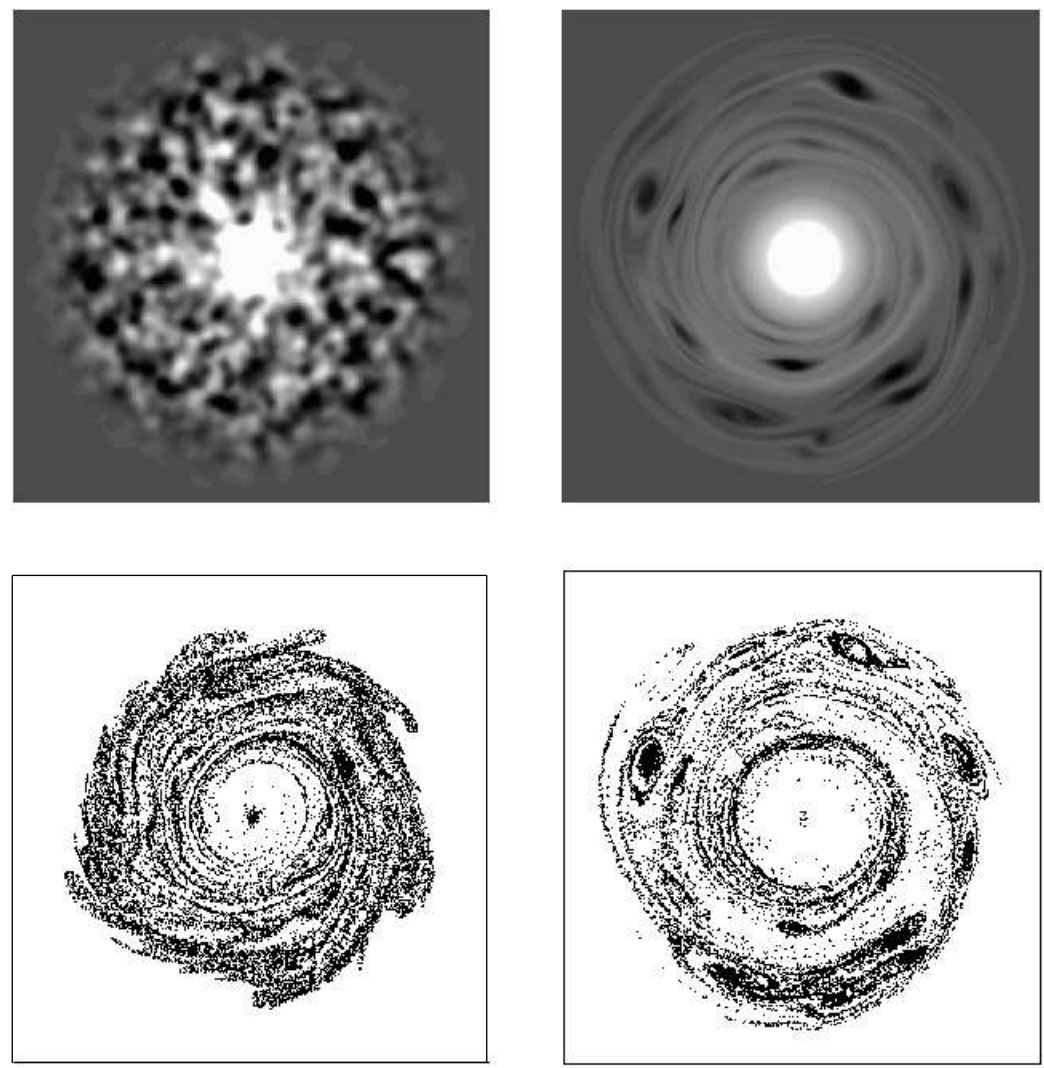

Fig. 2. A scenario of planet formation inside large-scale vortices presumably present in the Keplerian gaseous disk surrounding a star at its birth. Starting from a random vorticity field, a series of anticyclonic vortices appears spontaneously (upper panel). Due to the Coriolis force and to the friction with the gas, these vortices can efficiently trap dust particles passing nearby (lower pannel). The local increase of dust concentration inside the vortices can initiate the formation of planetesimals and planets by gravitational instability. This numerical simulation is taken from [15].

turbulent flows are not simply more probable than any other possible configuration, i.e. if they cannot be considered as maximum entropy states. This statistical mechanics approach has been initiated by Onsager [101] for a system of point vortices and by Chandrasekhar 21] in the case of self-gravitating systems.

It turns out that the statistical mechanics of two-dimensional vortices and self-gravitating systems present a deep analogy despite the very different physical nature of these systems. This analogy was pointed out by Chavanis in 29, 32, 35. and further developed in $54,30,34,36,47,48]$. In the following, we will essentially discuss the statistical mechanics of $2 \mathrm{D}$ vortices and refer to the review of Padmanabhan [103] (and his contribution in this book) for more details about the 


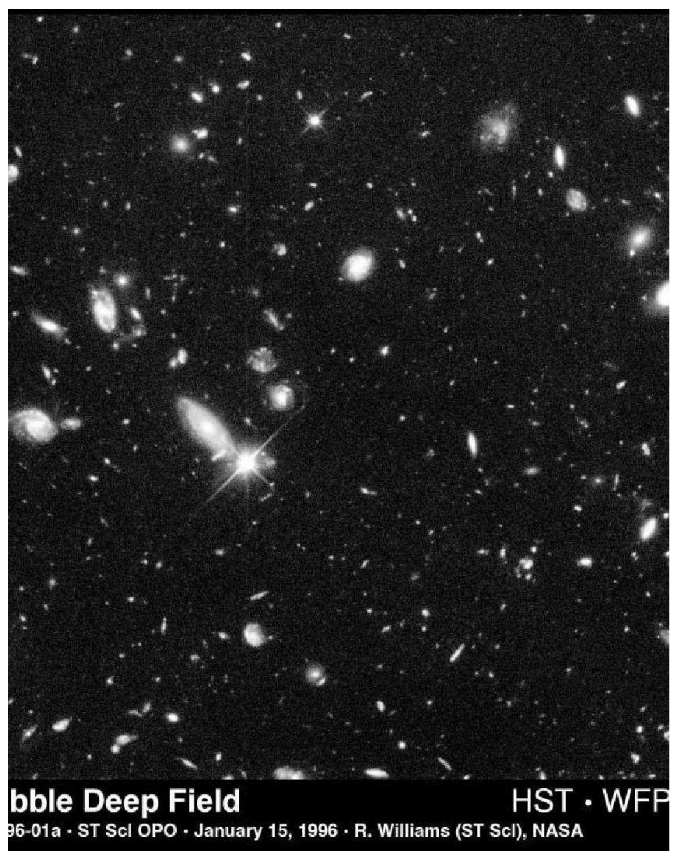

Fig. 3. Large-scale structures in the universe as observed with the Hubble space telescope. The analogy with Fig. 1 is striking and will be discussed in detail in this paper.

statistical mechanics of self-gravitating systems. We will see that the analogy between two-dimensional vortices and (three-dimensional) self-gravitating systems concerns not only the prediction of the equilibrium state, i.e. the formation of large-scale structures, but also the statistics of fluctuations and the relaxation towards equilibrium.

This paper is organized as follows. In Sec. 2, we discuss the statistical mechanics of point vortices introduced by Onsager 101 and further developed by Joyce \& Montgomery [70] and Pointin \& Lundgren 107 among others (see a complete list of references in the book of Newton 98$]$ ). We discuss the existence of a thermodynamic limit in Sec. 2.7 and make the connexion with field theory. Statistical equilibrium states of axisymmetric flows are obtained analytically in Sec. 2.8 2.9. The relation with equilibrium states of self-gravitating systems is shown in Sec. 2.10. In Sec. 3, we discuss the statistics of velocity fluctuations produced by a random distribution of point vortices and use this stochastic approach to obtain an estimate of the diffusion coefficient of point vortices. Application to $2 \mathrm{D}$ decaying turbulence is considered in Sec. 3.4. In Sec. 1, we describe the relaxation of a point vortex in a thermal bath and analyze this relaxation in terms of a Fokker-Planck equation involving a diffusion and a drift. In Sec. 5, we develop a more general kinetic theory of point vortices. A new kinetic equation is obtained which satisfies all conservation laws of the point vortex system and increases the Boltzmann entropy (H-theorem). We mention the connexion with 
the kinetic theory of stars developed by Chandrasekhar [21]. In Sec. 6, we discuss the violent relaxation of $2 \mathrm{D}$ vortices and stellar systems. We mention the analogy between the Vlasov and the Euler equations and between the statistical approach developed by Lynden-Bell [90] for collisionless stellar systems and by Kuz'min [83, Miller [95] and Robert \& Sommeria 111] for continuous vorticity fields. The concepts of "chaotic mixing" and "incomplete relaxation" are discussed in the light of a relaxation theory in Sec. 6.3. Application of statistical mechanics to geophysical flows and Jupiter's Great Red Spot are evocated in Sec. 6.4.

\section{Statistical mechanics of point vortices in two-dimensional hydrodynamics}

\subsection{Two-dimensional perfect flows}

The equations governing the dynamics of an invisicid flow are the equation of continuity and the Euler equation:

$$
\begin{gathered}
\frac{\partial \rho}{\partial t}+\nabla(\rho \mathbf{u})=0, \\
\frac{\partial \mathbf{u}}{\partial t}+(\mathbf{u} \nabla) \mathbf{u}=-\frac{1}{\rho} \nabla p .
\end{gathered}
$$

For an incompressible flow, the equation of continuity reduces to the condition

$$
\nabla \mathbf{u}=0
$$

If, in addition, the flow is two-dimensional, this last equation can be written $\partial_{x} u+\partial_{y} v=0$, where $(u, v)$ are the components of the velocity. According to the Schwarz theorem, there exists a streamfunction $\psi$ such that $u=\partial_{y} \psi, v=-\partial_{x} \psi$, or, equivalently

$$
\mathbf{u}=-\mathbf{z} \times \nabla \psi,
$$

where $\mathbf{z}$ is a unit vector normal to the flow. The vorticity

$$
\boldsymbol{\omega}=\nabla \times \mathbf{u}=\omega \mathbf{z}, \quad \text { with } \quad \omega=\partial_{x} v-\partial_{y} u,
$$

is directed along the vertical axis. According to the Stokes formula, the circulation of the velocity along a closed curve $(C)$ delimiting a domain area $(S)$ is

$$
\Gamma=\oint_{(C)} \mathbf{u} d \mathbf{l}=\int_{(S)} \omega d^{2} \mathbf{r} .
$$

Taking the curl of Eq. (4), we find that the vorticity is related to the stream function by a Poisson equation

$$
\Delta \psi=-\omega
$$


where $\Delta=\partial_{x x}^{2}+\partial_{y y}^{2}$ is the Laplacian operator. In an unbounded domain, this equation can be written in integral form as

$$
\psi(\mathbf{r}, t)=-\frac{1}{2 \pi} \int \omega\left(\mathbf{r}^{\prime}, t\right) \ln \left|\mathbf{r}-\mathbf{r}^{\prime}\right| d^{2} \mathbf{r}^{\prime},
$$

and the velocity field can be expressed in terms of the vorticity as

$$
\mathbf{u}(\mathbf{r}, t)=\frac{1}{2 \pi} \mathbf{z} \times \int \omega\left(\mathbf{r}^{\prime}, t\right) \frac{\mathbf{r}-\mathbf{r}^{\prime}}{\left|\mathbf{r}-\mathbf{r}^{\prime}\right|^{2}} d^{2} \mathbf{r}^{\prime} .
$$

In a bounded domain, Eq. (8) must be modified so as to take into account vortex images. The impermeability condition implies that $\psi$ is constant on the boundary and we shall take $\psi=0$ by convention. Taking the curl of Eq. (2), the pressure term disapears and the Euler equation becomes

$$
\frac{\partial \omega}{\partial t}+\mathbf{u} \nabla \omega=0
$$

This corresponds to the transport of the vorticity $\omega$ by the velocity field $\mathbf{u}$. It is easy to show that the flow conserves the kinetic energy

$$
E=\int \frac{\mathbf{u}^{2}}{2} d^{2} \mathbf{r}
$$

Using Eqs. (4) (77), one has successively

$$
E=\frac{1}{2} \int(\nabla \psi)^{2} d^{2} \mathbf{r}=\frac{1}{2} \int \psi(-\Delta \psi) d^{2} \mathbf{r}=\frac{1}{2} \int \omega \psi d^{2} \mathbf{r},
$$

where the second equality is obtained by a part integration with the condition $\psi=0$ on the boundary. Therefore, $E$ can be interpreted either as the kinetic energy of the flow (see Eq. (11)) or as a potential energy of interaction between vortices (see Eq. (12)).

\subsection{The point vortex gas}

We shall consider the situation in which the velocity is created by a collection of $N$ point vortices. In that case, the vorticity field can be expressed as a sum of $\delta$-functions in the form

$$
\omega(\mathbf{r}, t)=\sum_{i=1}^{N} \gamma_{i} \delta\left(\mathbf{r}-\mathbf{r}_{i}(t)\right),
$$

where $\mathbf{r}_{i}(t)$ denotes the position of point vortex $i$ at time $t$ and $\gamma_{i}$ is its circulation. According to Eqs. (9) (13), the velocity of a point vortex is equal to the sum of the velocities $\mathbf{V}(j \rightarrow i)$ produced by the $N-1$ other vortices, i.e.

$$
\mathbf{V}_{i}=\sum_{j \neq i} \mathbf{V}(j \rightarrow i) \quad \text { with } \quad \mathbf{V}(j \rightarrow i)=-\frac{\gamma_{j}}{2 \pi} \mathbf{z} \times \frac{\mathbf{r}_{j}-\mathbf{r}_{i}}{\left|\mathbf{r}_{j}-\mathbf{r}_{i}\right|^{2}}
$$


As emphasized by Kirchhoff [79], the above dynamics can be cast in a Hamiltonian form

$$
\begin{gathered}
\gamma_{i} \frac{d x_{i}}{d t}=\frac{\partial H}{\partial y_{i}}, \quad \gamma_{i} \frac{d y_{i}}{d t}=-\frac{\partial H}{\partial x_{i}}, \\
H=-\frac{1}{2 \pi} \sum_{i<j} \gamma_{i} \gamma_{j} \ln \left|\mathbf{r}_{i}-\mathbf{r}_{j}\right|
\end{gathered}
$$

where the coordinates $(x, y)$ of the point vortices are canonically conjugate. These equations of motion still apply when the fluid is restrained by boundaries, in which case the Hamiltonian (16) is modified so as to allow for vortex images, and may be constructed in terms of Green's functions depending on the geometry of the domain. Since $H$ is not explicitly time dependant, it is a constant of the motion and it represents the "potential" energy of the point vortices. The other conserved quantities are the angular momentum $\sum_{i} \gamma_{i} r_{i}^{2}$ and the impulse $\sum_{i} \gamma_{i} \mathbf{r}_{i}$. Note that the Hamiltonian (16) does not involve a "kinetic" energy of the point vortices in the usual sense (i.e., a quadratic term $\sum_{i} \frac{p_{i}^{2}}{2 m}$ ). This is related to the particular circumstance that a point vortex is not a material particle. Indeed, an isolated vortex remains at rest contrary to a material particle which has a rectilinear motion due to its inertia. Point vortices form therefore a very peculiar Hamiltonian system. Note also that the Hamiltonian of point vortices can be either positive or negative (in the case of vortices of different signs) whereas the kinetic energy of the flow is necessarily positive. This is clearly a drawback of the point vortex model.

\subsection{The microcanonical approach of Onsager (1949)}

The statistical mechanics of point vortices was first considered by Onsager 101 who showed the existence of negative temperature states at which point vortices of the same sign cluster into "supervortices". He could therefore explain the formation of large, isolated vortices in nature. This was a remarkable anticipation since observations were very scarce at that time.

Let us consider a liquid enclosed by a boundary, so that the vortices are confined to an area $A$. Since the coordinates $(x, y)$ of the point vortices are canonically conjugate, the phase space coincides with the configuration space and is finite:

$$
\int d x_{1} d y_{1} \ldots d x_{N} d y_{N}=\left(\int d x d y\right)^{N}=A^{N} .
$$

This striking property contrasts with most classical Hamiltonian systems considered in statistical mechanics which have unbounded phase spaces due to the presence of a kinetic term in the Hamiltonian.

As is usual in the microcanonical description of a system of $N$ particles, we introduce the density of states

$$
g(E)=\int d x_{1} d y_{1} \ldots d x_{N} d y_{N} \delta\left(E-H\left(x_{1}, y_{1}, \ldots, x_{N}, y_{N}\right)\right),
$$


which gives the phase space volume per unit interaction energy $E$. The equilibrium $N$-body distribution of the system, satisfying the normalization condition $\int \mu\left(\mathbf{r}_{1}, \ldots, \mathbf{r}_{N}\right) d^{2} \mathbf{r}_{1} \ldots d^{2} \mathbf{r}_{N}=1$, is given by

$$
\mu\left(\mathbf{r}_{1}, \ldots, \mathbf{r}_{N}\right)=\frac{1}{g(E)} \delta\left(E-H\left(\mathbf{r}_{1}, \ldots, \mathbf{r}_{N}\right)\right)
$$

This formula simply means that, in the microcanonical ensemble, all accessible microstates (having the required energy $E$ ) are equiprobable at statistical equilibrium.

The phase space volume which corresponds to energies $H\left(\mathbf{r}_{1}, \ldots, \mathbf{r}_{N}\right)$ less than a given value $E$ can be written

$$
\Phi(E)=\int_{E_{\min }}^{E} g(E) d E
$$

It increases monotonically from zero to $A^{N}$ when $E$ goes from $E_{\text {min }}$ to $+\infty$. Therefore, $g(E)=d \Phi(E) / d E$ will have a maximum value at some $E=E_{m}$, say, before decreasing to zero when $E \rightarrow+\infty$. by

In the microcanonical ensemble, the entropy and the temperature are defined

$$
S=\ln g(E), \quad \beta=\frac{1}{T}=\frac{d S}{d E} .
$$

For $E>E_{m}, S(E)$ is a decreasing function of energy and consequently the temperature is negative. Now, high energy states $E \gg E_{m}$ are clearly those in which the vortices of the same sign are crowded as close together as possible. For energies only slighlty greater than $E_{m}$, the concentration will not be so dramatic but there will be a tendency for the vortices to group themselves together on a macroscopic scale and form "clusters" or "supervortices". By contrast, for $E<E_{m}$, the temperature is positive and the vortices have the tendency to accumulate on the boundary of the domain in order to decrease their energy. For a system with positive and negative vortices, the negative temperature states, achieved for relatively high energies, consist of two large counter-rotating vortices physically well separated in the box. On the contrary when $E \rightarrow-\infty$, the temperature is positive and vortices of opposite circulation tend to pair off.

\subsection{The equation of state}

For a two-dimensional gas of particles interacting via a Coulombian potential in $\ln r$, the equation of state can be derived exactly. Let us assume that the system is enclosed in a domain of surface $V=R^{2}$. The density of states can be written

$$
g(E, V)=\int_{0}^{R} \ldots \int_{0}^{R} \prod_{i=1}^{N} d^{2} \mathbf{r}_{i} \delta\left(E+\frac{1}{2 \pi} \sum_{i<j} \gamma_{i} \gamma_{j} \ln \left|\mathbf{r}_{i}-\mathbf{r}_{j}\right|\right)
$$


Making the change of variable $\mathbf{x}=\mathbf{r} / R$, we find that $g(E, V)=V^{N} g\left(E^{\prime}, 1\right)$ with $E^{\prime}=E+\frac{1}{4 \pi} \ln V \sum_{i<j} \gamma_{i} \gamma_{j}$. Therefore, the entropy satisfies $S(E, V)=$ $N \ln V+S\left(E^{\prime}, 1\right)$ and the pressure $P=T(\partial S / \partial V)_{E}$ is exactly given by

$$
P=\frac{N}{\beta V}\left(1+\frac{\beta}{4 \pi N} \sum_{i<j} \gamma_{i} \gamma_{j}\right) .
$$

If the vortices have the same circulation $\gamma$, we obtain

$$
P=\frac{N}{\beta V}\left(1+\frac{\gamma^{2}}{8 \pi}(N-1) \beta\right) .
$$

In particular the pressure vanishes for

$$
\beta_{c}=-\frac{8 \pi}{(N-1) \gamma^{2}}
$$

We shall see in Sec. 2.8 that this negative critical inverse temperature is the minimum inverse temperature that the system can achieve.

If, on the other hand, we consider a neutral system consisting of $N / 2$ vortices of circulation $\gamma$ and $N / 2$ vortices of circulation $-\gamma$, we find

$$
P=\frac{N}{\beta V}\left(1-\frac{\beta \gamma^{2}}{8 \pi}\right) \text {. }
$$

This result is well-known is plasma physics 113. The critical temperature at which the pressure vanishes is now positive

$$
\beta_{c}=\frac{8 \pi}{\gamma^{2}}
$$

and independant on the number of point vortices in the system. For $\beta>\beta_{c}$ the pressure is negative so this range of temperatures is forbidden. For simplicity, we have not taken into account the contribution of images in the previous calculations; this can slightly change the results but this should not alter the existence of the critical inverse temperatures reported above.

\subsection{The mean-field approximation}

It is easy to show that the exact distribution of point vortices (13) expressed in terms of $\delta$-functions is solution of the Euler equation (10). This is proved as follows. Taking the derivative of Eq. (13) with respect to time, we obtain

$$
\frac{\partial \omega}{\partial t}=-\sum_{i=1}^{N} \gamma \nabla \delta\left(\mathbf{r}-\mathbf{r}_{i}(t)\right) \mathbf{V}_{i}
$$

Since $\mathbf{V}_{i}=\mathbf{u}\left(\mathbf{r}_{i}(t), t\right)$, we can rewrite the foregoing equation in the form

$$
\frac{\partial \omega}{\partial t}=-\nabla \sum_{i=1}^{N} \gamma \delta\left(\mathbf{r}-\mathbf{r}_{i}(t)\right) \mathbf{u}(\mathbf{r}, t) .
$$


Since the velocity is divergenceless, we obtain

$$
\frac{\partial \omega}{\partial t}=-\mathbf{u}(\mathbf{r}, t) \nabla \sum_{i=1}^{N} \gamma \delta\left(\mathbf{r}-\mathbf{r}_{i}(t)\right)=-\mathbf{u} \nabla \omega .
$$

Therefore, in the point vortex model, the Euler equation (10) contains exactly the same information as the Hamiltonian system (15).

This description in terms of $\delta$-functions, while being technically correct, is useless for practical purposes because it requires the knowledge of the exact trajectories of the point vortices for an arbitrary initial condition or the solution of the Euler equation (10). When $N$ is large, this task is impossibly difficult. Therefore, instead of the exact vorticity field expressed in terms of $\delta$-functions, one is more interested by functions which are smooth. For that reason, we introduce a smooth vorticity field $\langle\omega\rangle(\mathbf{r}, t)$ which is proportional to the average number of vortices contained in the cell $(\mathbf{r}, \mathbf{r}+d \mathbf{r})$ at time $t$. This description requires that it is possible to divide the domain in a large number of cells in such a way that each cell is (a) large enough to contain a macroscopic number of point vortices but (b) small enough for all the particles in the cell can be assumed to possess the same average characteristics of the cell.

Formally, the average vorticity field is given by

$$
\langle\omega\rangle(\mathbf{r}, t)=\sum_{i=1}^{N} \gamma\left\langle\delta\left(\mathbf{r}-\mathbf{r}_{i}(t)\right)\right\rangle,
$$

where the statistical average of a function $X\left(\mathbf{r}_{1}, \ldots, \mathbf{r}_{N}\right)$ is defined by

$$
\langle X\rangle=\int \mu\left(\mathbf{r}_{1}, \ldots, \mathbf{r}_{N}, t\right) X\left(\mathbf{r}_{1}, \ldots, \mathbf{r}_{N}\right) d^{2} \mathbf{r}_{1} \ldots d^{2} \mathbf{r}_{N},
$$

where $\mu\left(\mathbf{r}_{1}, \ldots, \mathbf{r}_{N}, t\right)$ is the $N$-body distribution function of the system at time $t$. The average vorticity can be rewritten

$$
\langle\omega\rangle(\mathbf{r}, t)=N \gamma P(\mathbf{r}, t)=\gamma\langle n\rangle(\mathbf{r}, t),
$$

where we have introduced the one-vortex distribution function

$$
P\left(\mathbf{r}_{1}, t\right)=\int \mu\left(\mathbf{r}_{1}, \ldots, \mathbf{r}_{N}, t\right) d^{2} \mathbf{r}_{2} \ldots d^{2} \mathbf{r}_{N},
$$

and the local vortex density $\langle n\rangle=N P(\mathbf{r}, t)$. In the foregoing relations, we have implicitly used the fact that the vortices are identical. The average energy of the system is given by

$$
\begin{aligned}
& E=\langle H\rangle=-\frac{1}{4 \pi} \sum_{i \neq j} \gamma^{2}\left\langle\ln \left|\mathbf{r}_{i}-\mathbf{r}_{j}\right|\right\rangle \\
& =-\frac{1}{4 \pi} N(N-1) \gamma^{2} \int g\left(\mathbf{r}, \mathbf{r}^{\prime}, t\right) \ln \left|\mathbf{r}-\mathbf{r}^{\prime}\right| d^{2} \mathbf{r} d^{2} \mathbf{r}^{\prime},
\end{aligned}
$$


where

$$
g\left(\mathbf{r}_{1}, \mathbf{r}_{2}, t\right)=\int \mu\left(\mathbf{r}_{1}, \ldots, \mathbf{r}_{N}, t\right) d^{3} \mathbf{r}_{2} \ldots d^{2} \mathbf{r}_{N}
$$

is the two-body distribution function. In the mean-field approximation, which is exact in a properly defined thermodynamic limit with $N \rightarrow+\infty$ (see Sec. 2.7), we have

$$
g\left(\mathbf{r}_{1}, \mathbf{r}_{2}, t\right)=P\left(\mathbf{r}_{1}, t\right) P\left(\mathbf{r}_{2}, t\right) .
$$

Accounting that $N(N-1) \simeq N^{2}$ for large $N$, the average energy takes the form

$$
E=-\frac{1}{4 \pi} \int\langle\omega\rangle(\mathbf{r}, t)\langle\omega\rangle\left(\mathbf{r}^{\prime}, t\right) \ln \left|\mathbf{r}-\mathbf{r}^{\prime}\right| d^{2} \mathbf{r} d^{2} \mathbf{r}^{\prime}
$$

Using Eq. (8), the expression (38) for $E$ can be rewritten

$$
E=\frac{1}{2} \int\langle\omega\rangle \psi d^{2} \mathbf{r}=\int \frac{\langle\mathbf{u}\rangle^{2}}{2} d^{2} \mathbf{r}
$$

where $\psi$ is the streamfunction created by the average vorticity $\langle\omega\rangle$ and where $\langle\mathbf{u}\rangle$ is the smooth velocity field.

\subsection{The maximum entropy state}

We now wish to determine the equilibrium distribution of vortices following a statistical mechanics approach. Using Boltzmann procedure, we divide the domain in macrocells with area $\Delta$. Let $n_{i}$ denote the number of point vortices in the cell $\Delta_{i}$. We now decompose each macrocell into $\nu$ microcells with equal area. A macrostate is determined by the distribution $\left\{n_{i}\right\}$. Several configurations can lead to the same macrostate: each of them will be called a microstate. Using a combinatorial analysis, the number of microstates corresponding to the macrostate $\left\{n_{i}\right\}$ is

$$
W\left(\left\{n_{i}\right\}\right)=N ! \prod_{i} \frac{\nu^{n_{i}}}{n_{i} !} .
$$

The logarithm of this number defines the Boltzmann entropy. Using Stirling formula and considering the continuum limit in which $\Delta, \nu \rightarrow 0$, we get the classical formula

$$
S=-N \int P(\mathbf{r}) \ln P(\mathbf{r}) d^{2} \mathbf{r}
$$

where $P(\mathbf{r})$ is the density probability that a point vortex be found in the surface element centered on $\mathbf{r}$. At equilibrium, the system is in the most probable macroscopic state, i.e. the state that is the most represented at the microscopic level. This optimal state is obtained by maximizing the Boltzmann entropy (41) at fixed energy (39) and vortex number $N$, or total circulation

$$
\Gamma=N \gamma=\int\langle\omega\rangle d^{2} \mathbf{r}
$$


Writing the variational principle in the form

$$
\delta S-\beta \delta E-\alpha \delta \Gamma=0,
$$

where $\beta$ and $\alpha$ are Lagrange multipliers, it is readily found that the maximum entropy state corresponds to the Boltzmann distribution

$$
\langle\omega\rangle=A e^{-\beta \gamma \psi},
$$

with inverse temperature $\beta$. We can account for the conservation of angular momentum $L=\int\langle\omega\rangle r^{2} d^{2} \mathbf{r}$ (in a circular domain) and impulse $P=\int\langle\omega\rangle y d^{2} \mathbf{r}$ (in a channel) by introducing appropriate Lagrange multipliers $\Omega$ and $U$ for each of these constraints. In that case, Eq. (44) remains valid provided that we replace the streamfunction $\psi$ by the relative streamfunction $\psi^{\prime}=\psi+\frac{\Omega}{2} r^{2}-U y$. Substituting the Boltzmann relation between $\langle\omega\rangle$ and $\psi$ in the Poisson equation (『), we obtain a differential equation for the streamfunction

$$
-\Delta \psi=A e^{-\beta \gamma \psi},
$$

which determines the statistical equilibrium distribution of vortices. This Boltzmann Poisson equation can be easily generalized to include a spectrum of circulations among the vortices.

The combinatorial analysis presented in this section was performed by Joyce \& Montgomery [70]. The mean-field equation (45) was also obtained by Pointin \& Lundgren 107] from the equilibrium BGK hierarchy. In fact, this mean-field approach was first developed by Onsager but his results were not published (U. Frisch, private communication).

\subsection{Field theory and thermodynamic limit}

Let us consider a collection of $N$ point vortices with equal circulation $\gamma$ in a bounded domain of size $R$. We want to give a rational to the mean-field approximation considered previously by defining a proper thermodynamic limit for point vortices. Rigorous results have been established in [17,58. In the following, we present a less rigorous, albeit equivalent, field theory approach inspired by the work of 65, 103, 57 for self-gravitating systems.

In the microcanonical ensemble, the quantity of fundamental interest is the density of states

$$
g(E)=\int \delta\left(E-H\left(\mathbf{r}_{1}, \ldots, \mathbf{r}_{N}\right)\right) \prod_{i=1}^{N} d^{2} \mathbf{r}_{i},
$$

which is explicitly given by

$$
g(E)=\int_{0}^{R} \ldots \int_{0}^{R} \prod_{i=1}^{N} d^{2} \mathbf{r}_{i} \delta\left(E+\frac{\gamma^{2}}{2 \pi} \sum_{i<j} \ln \frac{\left|\mathbf{r}_{i}-\mathbf{r}_{j}\right|}{R}\right),
$$


where the potential of interaction has been normalized by $R$. For simplicity, we have ignored the contribution of the images but this shall not affect the final results. We now introduce the change of variables $\mathbf{x}=\mathbf{r} / R$ and define the function

$$
u\left(\mathbf{x}_{1}, \ldots, \mathbf{x}_{N}\right)=\frac{1}{N} \sum_{i<j} \ln \left|\mathbf{x}_{i}-\mathbf{x}_{j}\right|
$$

and the dimensionless energy

$$
\Lambda=\frac{2 \pi E}{N^{2} \gamma^{2}} .
$$

In terms of these quantities, the density of states can be rewritten

$$
g(E)=\frac{2 \pi V^{N}}{N^{2} \gamma^{2}} \int_{0}^{1} \ldots \int_{0}^{1} \prod_{i=1}^{N} d^{2} \mathbf{x}_{i} \delta\left(\Lambda+\frac{1}{N} u\left(\mathbf{x}_{1}, \ldots, \mathbf{x}_{N}\right)\right) .
$$

The proper thermodynamic limit for a system of point vortices with equal circulation in the microcanonical ensemble is such that $N \rightarrow+\infty$ with fixed $\Lambda$. We see that the box size $R$ does not enter in the normalized energy $\Lambda$. Therefore, the thermodynamic limit corresponds to $N \rightarrow+\infty$ with $\gamma \sim N^{-1} \rightarrow 0$ and $E \sim 1$. This is a very unusual thermodynamic limit due to the non-extensivity of the system. Note that the total circulation $\Gamma=N \gamma$ remains fixed in this process.

For sufficiently large $N$, the density of states can be written

$$
\left.g(E) \simeq \int \mathcal{D} \rho e^{N S[\rho]} \delta(\Lambda-E[\rho])\right) \delta\left(1-\int \rho(\mathbf{r}) d^{2} \mathbf{r}\right)
$$

with

$$
\begin{gathered}
S[\rho]=-\int \rho(\mathbf{r}) \ln \rho(\mathbf{r}) d^{2} \mathbf{r}, \\
E[\rho]=-\frac{1}{2} \int \rho(\mathbf{r}) \rho\left(\mathbf{r}^{\prime}\right) \ln \left|\mathbf{r}-\mathbf{r}^{\prime}\right| d^{2} \mathbf{r} d^{2} \mathbf{r}^{\prime} .
\end{gathered}
$$

In the above formula, $g(E)$ has been expressed as a functional integral over the macrostates recalling that $e^{N S[\rho]}=W(\{\rho\})$ gives the number of microstates corresponding to the macrostate $\rho(\mathbf{r})$ (see Sec. 2.6). The crucial point to realize is that the vortex number $N$ appears explicitly in the exponential, all other terms being of order unity. Therefore, at the thermodynamic limit $N \rightarrow+\infty$, the functional integral is dominated by the distribution $\rho_{*}(\mathbf{r})$ which maximizes the Boltzmann entropy (52) under the constraints of fixed circulation and energy brought by the $\delta$-functions. In this limit, the mean-field approximation is exact and we have

$$
g(E)=e^{N S\left[\rho_{*}\right]}, \quad S(E)=N S\left[\rho_{*}\right] .
$$

In the canonical ensemble, the object of interest is the partition function

$$
Z(\beta)=\int e^{-\beta H\left(\mathbf{r}_{1}, \ldots, \mathbf{r}_{N}\right)} \prod_{i=1}^{N} d^{2} \mathbf{r}_{i}
$$


which is the normalization factor in the Gibbs measure

$$
\mu\left(\mathbf{r}_{1}, \ldots, \mathbf{r}_{N}\right)=\frac{1}{Z} e^{-\beta H\left(\mathbf{r}_{1}, \ldots, \mathbf{r}_{N}\right)} .
$$

The free energy is defined by the relation

$$
F(\beta)=-\frac{1}{\beta} \ln Z .
$$

Using the notations introduced previously, we can rewrite the integral (55) in the form

$$
Z(\beta)=V^{N} \int_{0}^{N} \ldots \int_{0}^{N} \prod_{i=1}^{N} d^{2} \mathbf{x}_{i} e^{\eta u\left(\mathbf{x}_{1}, \ldots, \mathbf{x}_{N}\right)},
$$

where

$$
\eta=\frac{\beta N \gamma^{2}}{2 \pi},
$$

is the normalized inverse temperature. It plays the role of the "plasma parameter" in plasma physics. It can be shown that the partition function (58) is convergent only for $\eta>\eta_{c}=-4 N /(N-1)$ which is consistent with the result (25). The proper thermodynamic limit for a canonical distribution of point vortices is such that $N \rightarrow+\infty$ with fixed $\eta$. In this limit, $\gamma \sim N^{-1}$ and $\beta \sim N$.

The partition function is related to the density of states by the Laplace transform

$$
Z(\beta)=\int_{-\infty}^{+\infty} d E g(E) e^{-\beta E}
$$

Therefore, for large $N$ one has, using Eq. (51),

$$
Z(\beta) \simeq \int \mathcal{D} \rho e^{N J[\rho]} \delta\left(1-\int \rho(\mathbf{r}) d^{2} \mathbf{r}\right),
$$

with

$$
J[\rho]=S[\rho]-\eta E[\rho] .
$$

At the thermodynamic limit, the functional integral is dominated by the distribution $\rho_{*}(\mathbf{r})$ which maximizes the Massieu function $J[\rho]$ under the constraint of a fixed circulation. In this limit, the mean-field approximation is exact and we have

$$
Z(\beta)=e^{N J\left[\rho_{*}\right]}, \quad F=E-T S .
$$

It can be noted that the critical points of entropy at fixed energy and circulation (microcanonical description) and the critical points of free energy at fixed temperature and circulation (canonical description) coincide. This is not necessarily the case for the second order variations of entropy and free energy. Therefore, a distribution $\rho(\mathbf{r})$ can be a maximum of $S[\rho]$ but a minimum (or a saddle point) of $J[\rho]$. In that case, the equilibrium is stable in the microcanonical ensemble but unstable in the canonical ensemble (and the procedure leading to Eq. (63) is clearly not valid). When this situation happens, the ensembles are inequivalent 
and phase transitions occur. This is the case, in particular, for the gravitational problem (see Sec. 2.10).

Finally, the grand canonical partition function is defined by

$$
Z_{G C}=\sum_{N=0}^{+\infty} \frac{z^{N}}{N !} \int \prod_{i=1}^{N} d^{2} \mathbf{r}_{i} e^{-\beta H_{N}\left(\mathbf{r}_{1}, \ldots, \mathbf{r}_{N}\right)},
$$

where $z$ is the fugacity. Using the Hubbard-Stratanovich transformation

$$
\int \mathcal{D} \xi e^{-\int\left\{\frac{1}{2}(\nabla \xi)^{2}-\rho(\mathbf{r}) \xi(\mathbf{r})\right\} d^{2} \mathbf{r}}=e^{-\frac{1}{2} \int \rho(\mathbf{r}) G\left(\mathbf{r}-\mathbf{r}^{\prime}\right) \rho\left(\mathbf{r}^{\prime}\right) d^{2} \mathbf{r} d^{2} \mathbf{r}^{\prime}},
$$

where $G$ is the Green function of the Laplacian operator, we can rewrite the Boltzmann factor in the form

$$
e^{-\beta H_{N}}=\int \mathcal{D} \xi e^{-\int \frac{1}{2}(\nabla \xi)^{2} d^{2} \mathbf{r}+\sqrt{-\beta} \sum_{i=1}^{N} \gamma \xi\left(\mathbf{r}_{i}\right)},
$$

where we have assumed $\beta<0$ which is the case of physical interest. Substituting this result in Eq. (64) and introducing the notation $\phi(\mathbf{r})=\sqrt{-\beta} \gamma \xi(\mathbf{r})$, we can easily carry out the summation on $N$ to obtain

$$
\begin{gathered}
Z_{G C}=\int \mathcal{D} \phi e^{-\frac{1}{T_{e f f}} \int d^{2} \mathbf{r}\left\{\frac{1}{2}(\nabla \phi)^{2}-\mu^{2} e^{\phi}(\mathbf{r})\right\}}, \\
T_{e f f}=-\beta \gamma^{2}, \quad \mu^{2}=-z \beta \gamma^{2} .
\end{gathered}
$$

Therefore, the grand partition function of the point vortex gas corresponds to a Liouville field theory with an action

$$
S[\phi]=\frac{1}{T_{\text {eff }}} \int d^{2} \mathbf{r}\left\{\frac{1}{2}(\nabla \phi)^{2}-\mu^{2} e^{\phi}(\mathbf{r})\right\} .
$$

While the previous description is formally correct if we define $Z$ and $Z_{G C}$ by Eqs. (55) and (64), it must be noted however that the canonical and grand canonical ensembles may not have a physical meaning for point vortices. In particular, it is not clear how one can impose a thermal bath at negative temperature. On the other hand, the usual procedure to derive the canonical ensemble from the microcanonical ensemble rests on a condition of additivity which is clearly lacking in the present case.

\subsection{Axisymmetric equilibrium states in a disk}

Let us consider a collection of $N$ point vortices with circulation $\gamma$ confined within a disk of radius $R$. At statistical equilibrium, the streamfunction $\psi$ is solution of the Boltzmann-Poisson equation (45). If we work in a circular domain, we must in principle account for the conservation of angular momentum. This can lead to bifurcations between axisymmetric and off-axis solutions [117]. We shall, 
however, ignore this constraint for the moment in order to obtain analytical expressions for the thermodynamical parameters. This is a sufficient approximation to illustrate the structure of the problem, which is our main concern here.

If we confine our attention to axisymmetric solutions, the Boltzmann-Poisson equation (45) can be written

$$
\begin{gathered}
\frac{1}{r} \frac{d}{d r}\left(r \frac{d \psi}{d r}\right)=-\omega_{0} e^{-\beta \gamma\left(\psi-\psi_{0}\right)} . \\
\psi^{\prime}(0)=0, \quad \psi(R)=0,
\end{gathered}
$$

where $\psi_{0}$ and $\omega_{0}$ are the values of streamfunction and vorticity at $r=0$. Introducing the function $\phi=\beta \gamma\left(\psi-\psi_{0}\right)$ and the dimensionless radial distance $\xi=\left(|\beta| \gamma \omega_{0}\right)^{1 / 2} r$, Eq. (70) can be reduced to the form

$$
\begin{gathered}
\frac{1}{\xi} \frac{d}{d \xi}\left(\xi \frac{d \phi}{d \xi}\right)=\lambda e^{-\phi}, \\
\phi(0)=\phi^{\prime}(0)=0,
\end{gathered}
$$

with $\lambda=1$ if $\beta<0$ and $\lambda=-1$ if $\beta>0$. It turns out that this equation can be solved analytically as noticed by a number of authors. With the change of variables $t=\ln \xi$ and $\phi=2 \ln \xi-z$, Eq. (72) can be rewritten

$$
\frac{d^{2} z}{d t^{2}}=-\lambda e^{z}=-\frac{d}{d z}\left(\lambda e^{z}\right)
$$

This corresponds to the motion of a ficticious particle in a potential $V(z)=\lambda e^{z}$. This equation is readily integrated and, returning to original variables, we finally obtain

$$
e^{-\phi}=\frac{1}{\left(1+\frac{\lambda}{8} \xi^{2}\right)^{2}}
$$

From the circulation theorem (6) applied to an axisymmetric flow, we have

$$
-\frac{d \psi}{d r}(r)=\frac{\Gamma(r)}{2 \pi r}
$$

where $\Gamma(r)=\int_{0}^{r} \omega\left(r^{\prime}\right) 2 \pi r^{\prime} d r^{\prime}$ is the circulation within $r$. Taking $r=R$ and introducing the dimensionless variables previously defined, we obtain

$$
\eta \equiv \frac{\beta \gamma \Gamma}{2 \pi}=-\alpha \phi^{\prime}(\alpha)
$$

where $\alpha=\left(|\beta| \gamma \omega_{0}\right)^{1 / 2} R$ is the value of $\xi$ at the box radius. Equation (77) relates $\alpha$ to the inverse temperature $\eta$. Using Eqs. (75) and (77), the vorticity field can be written explicitly

$$
\langle\omega\rangle(r)=\frac{4 \Gamma}{\pi R^{2}(\eta+4)} \frac{1}{\left(1-\frac{\eta}{\eta+4} \frac{r^{2}}{R^{2}}\right)^{2}} .
$$


At positive temperatures $(\eta>0)$, the vorticity is an increasing function of the distance and the vortices tend to accumulate on the boundary of the domain (Fig. 14. On the contrary at negative temperatures $(\eta<0)$, the vorticity is a decreasing function of the distance and the vortices tend to group themselves in the core of the domain to form a "supervortex" (Fig. 5). These results are consistent with Onsager's prediction 101. We also confirm that statistical equilibrium states only exist for $\eta>\eta_{c}=-4$, as previously discussed. At this critical temperature, the central vorticity becomes infinite and the solution tends to a Dirac peak:

$$
\langle\omega\rangle(\mathbf{r}) \rightarrow \Gamma \delta(\mathbf{r}), \quad \text { for } \quad \eta \rightarrow \eta_{c}=-4 .
$$

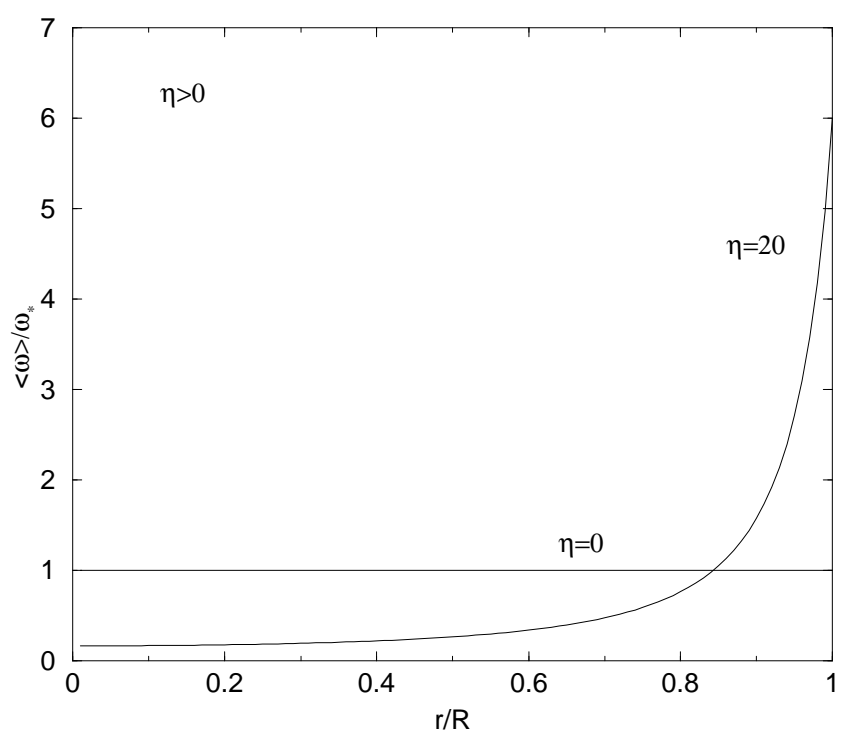

Fig. 4. Statistical equilibrium states of point vortices at positive temperatures $\left(\omega_{*}=\right.$ $\left.\Gamma / \pi R^{2}\right)$. The vortices are preferentially localized near the wall.

The energy defined by Eq. (39) can be written in the dimensionless form

$$
\Lambda \equiv \frac{2 \pi E}{\Gamma^{2}}=\frac{1}{2 \eta^{2}} \int_{0}^{\alpha} \phi^{\prime}(\xi)^{2} \xi d \xi
$$

The integral can be carried out explicitly using Eq. (75). Eliminating $\alpha$ between Eqs. (80) and (77), we find that the temperature is related to the energy by the equation of state

$$
\Lambda=\frac{1}{\eta}\left[\frac{4}{\eta} \ln \left(\frac{4}{4+\eta}\right)+1\right] .
$$

The corresponding equilibrium phase diagram is represented in Fig. 6. We check explicitly that the energy becomes infinite when $\eta \rightarrow \eta_{c}=-4$. The transition between positive and negative temperatures occurs for $\Lambda_{0}=1 / 8$. 


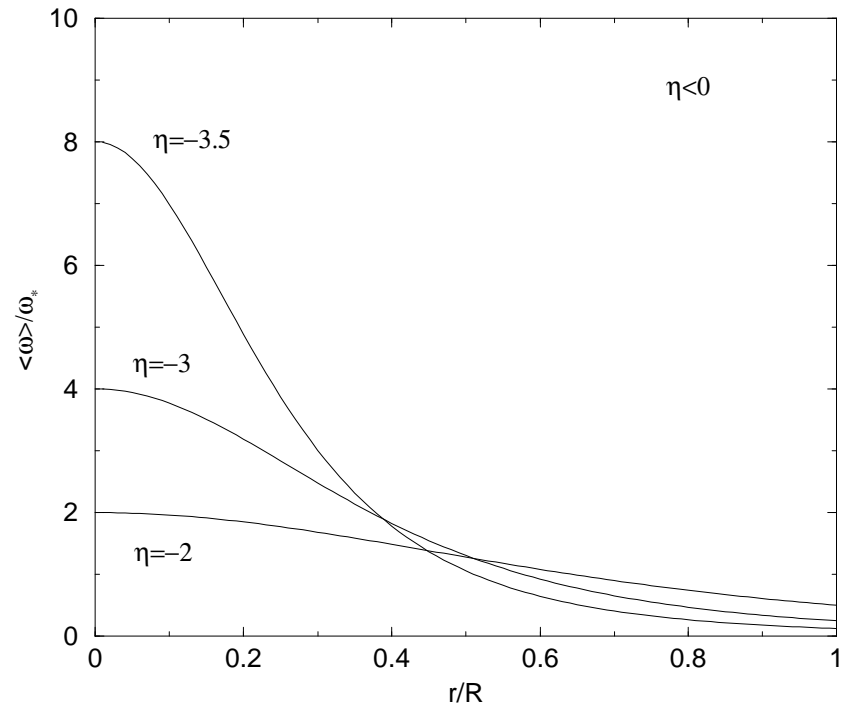

Fig. 5. Statistical equilibrium states of point vortices at negative temperatures showing a clustering. For $\eta=-4$, the vortices collapse at the center of the domain and the vorticity profile is a Dirac peak.

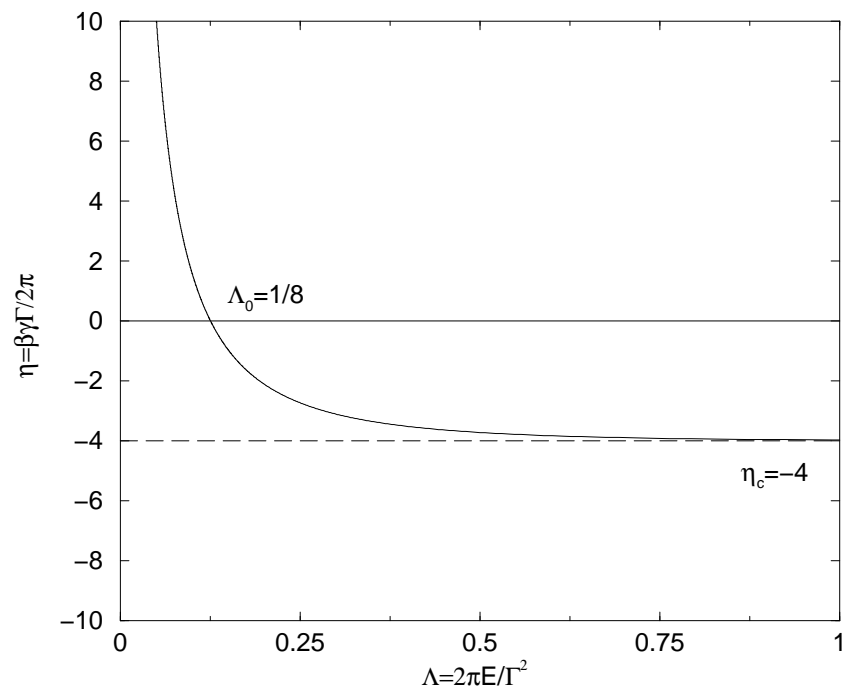

Fig. 6. Equilibrium phase diagram (caloric curve) for point vortices with equal circulation confined within a disk. For simplicity, the angular momentum has not been taken into account $(\Omega=0)$. 


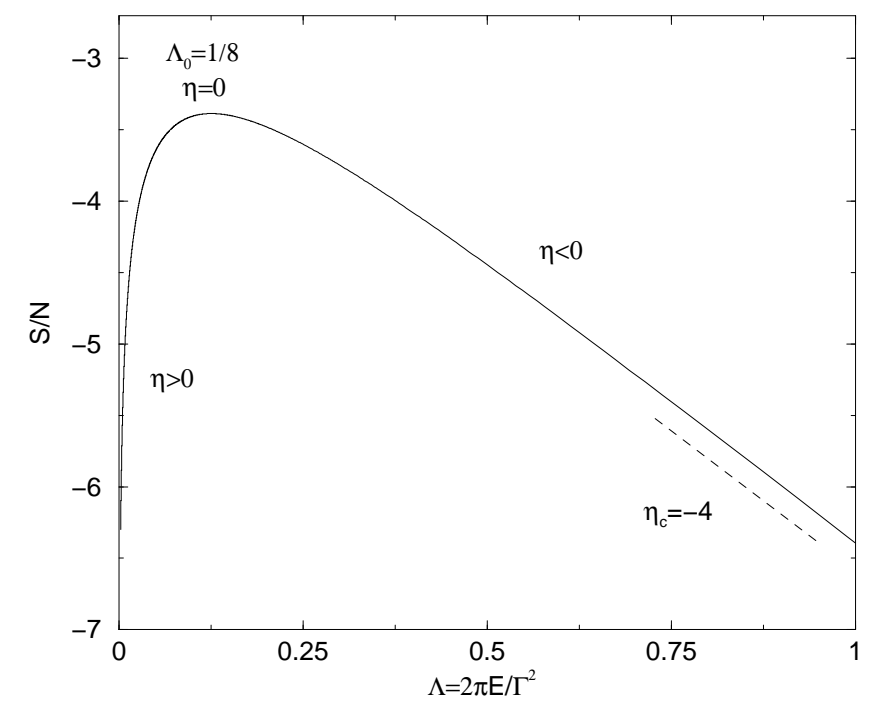

Fig. 7. Entropy vs energy plot for a system of point vortices with equal circulation confined within a disk.

The entropy (41) can also be calculated easily from the above results. Within an unimportant additive constant, it is given by

$$
\frac{S}{N}=\frac{8}{\eta} \ln 4-\left(1+\frac{8}{\eta}\right) \ln (4+\eta)
$$

Using Eq. (81), we can easily check that $d S / d E=\beta$, as it should. For $\Lambda<\Lambda_{0}=$ $1 / 8$, the entropy is an increasing function of energy (positive temperatures) and for $\Lambda>\Lambda_{0}$, it is a decreasing function of energy (negative temperatures). For $\Lambda \rightarrow 0, S / N \sim \ln \Lambda$ and for $\Lambda \rightarrow+\infty, S / N \sim-4 \Lambda$ (Fig. 7).

It is amusing to note that if we define a local pressure by the relation $p(\mathbf{r})=$ $\langle\omega\rangle / \gamma \beta$ (which is similar to the local equation of state $p=\rho T$ for an ideal gas of material particles), we find that the pressure $P$ at the boundary of the domain is exactly given by Eq. (24). Furthermore, using the Boltzmann distribution (44), we easily check that $\nabla p=-\langle\omega\rangle \nabla \psi$, which is similar to the equation of hydrostatic equilibrium for a fluid in a gravitational field. It is not clear whether these results bear more significance than is apparent at first sight.

\subsection{Equilibrium states in an unbounded domain}

In an infinite domain, it is necessary to take into account the conservation of angular momentum, because this constraint determines the typical size of the system. Furthermore, we shall assume that the vortices have the same sign otherwise positive and negative vortices will form dipoles and escape to infinity. Therefore, there is no equilibrium state in an infinite domain for a neutral system of point vortices. When the conservation of angular momentum is taken into 
account, the density of states is given by

$$
g(E, L)=\int \delta\left(E-H\left(\mathbf{r}_{1}, \ldots, \mathbf{r}_{N}\right)\right) \delta\left(L-\sum_{i=1}^{N} \gamma r_{i}^{2}\right) \prod_{i=1}^{N} d^{2} \mathbf{r}_{i},
$$

and the angular velocity of the flow by

$$
\Omega=2 T\left(\frac{\partial S}{\partial L}\right)_{E} .
$$

Using the same trick as in Sec. 2.4 with the change of variables $\mathbf{x}=\mathbf{r} / \sqrt{L}$, one finds the exact result

$$
\Omega=\frac{2 N}{\beta L}\left(1+\frac{\beta N \gamma^{2}}{8 \pi}\right) .
$$

Therefore, the vorticity field is determined by the Boltzmann distribution

$$
\langle\omega\rangle=A e^{-\beta \gamma \psi^{\prime}},
$$

where $\psi^{\prime}$ is the relative streamfunction

$$
\psi^{\prime} \equiv \psi+\frac{\Omega}{2} r^{2}=\psi+\frac{N}{4 \beta L}(4+\eta) r^{2} .
$$

For $\eta=0$, one has

$$
\langle\omega\rangle=\frac{\gamma N \Gamma}{\pi L} e^{-\frac{N \gamma}{L} r^{2}} .
$$

For large $r$, the asymptotic behavior of Eq. (86) is

$$
\langle\omega\rangle \sim \frac{1}{r^{-\eta}} e^{-\frac{N \gamma}{4 L}(4+\eta) r^{2}} \quad(r \rightarrow+\infty),
$$

where we have used $\psi \sim-(\Gamma / 2 \pi) \ln r$ at large distances. From Eq. (89), one sees that $\eta \geq-4$ is required for the existence of an integrable solution.

Inserting the relation (86) in the Poisson equation (7), we get

$$
-\Delta \psi^{\prime}=A e^{-\frac{2 \pi \eta}{N \gamma} \psi^{\prime}}-\frac{N^{2} \gamma^{2}}{2 \pi L \eta}(4+\eta) .
$$

With the change of variables

$$
\boldsymbol{\xi}=\left(\frac{\gamma N}{L}\right)^{1 / 2} \mathbf{r}, \quad \phi=\ln \left(\frac{L A}{N^{2} \gamma^{2}}\right)-\frac{2 \pi \eta}{N \gamma} \psi^{\prime},
$$

the Boltzmann-Poisson equation (90) can be written

$$
\frac{d^{2} \phi}{d \xi^{2}}+\frac{1}{\xi} \frac{d \phi}{d \xi}=2 \pi \eta e^{\phi}-(4+\eta),
$$


and the vorticity 86 ) becomes

$$
\langle\omega\rangle=\frac{N^{2} \gamma^{2}}{L} e^{\phi} .
$$

Equation (92) has been obtained by Lundgren \& Pointin [89] from the equilibrium BGK hierarchy. In the limit $\eta \rightarrow+\infty$, we have

$$
\langle\omega\rangle=\frac{\Gamma^{2}}{2 \pi L}, \quad \text { if } \quad r \leq(2 L / \Gamma)^{1 / 2},
$$

and $\langle\omega\rangle=0$ otherwise. This vortex patch is the state of minimum energy at fixed circulation and angular momentum. For $\eta \rightarrow-4$, one has approximately

$$
\langle\omega\rangle=\frac{N^{2} \gamma^{2}}{L} \frac{A}{\left(1-A \pi \eta \frac{\gamma N}{4 L} r^{2}\right)^{2}} e^{-\frac{\gamma N}{4 L}(4+\eta) r^{2}} .
$$

The first factor is an exact solution of Eq. (92) with the second term on the right hand side neglected (see Sec. 2.8). The second factor is a correction for large $r$, in agreement with the asymptotic result expressed by Eq. (89). The parameter $A$ tends to infinity as $\eta \rightarrow-4$ and is determined from the condition $\int\langle\omega\rangle d^{2} \mathbf{r}=\Gamma$ by the formula

$$
\pi A+\ln (\pi A)=-C-\ln \left(1+\frac{\eta}{4}\right)
$$

where $C=0.577 \ldots$ is the Euler constant. For $\eta=-4$, the vorticity profile is a Dirac peak and the energy tends to $+\infty$.

\subsection{The gravitational $N$-body problem}

It is interesting to compare the previous results with those obtained in the case of self-gravitating systems 103 . Formally, the structure of the $N$-vortex problem shares some analogies with the gravitational $N$-body problem. The force by unit of mass experienced by a star is given by

$$
\mathbf{F}_{i}=\sum_{j \neq i} \mathbf{F}(j \rightarrow i), \quad \mathbf{F}(j \rightarrow i)=G m \frac{\mathbf{r}_{j}-\mathbf{r}_{i}}{\left|\mathbf{r}_{j}-\mathbf{r}_{i}\right|^{3}}
$$

where $\mathbf{F}(j \rightarrow i)$ is the force created by star $j$ on star $i$. The force can be written as the gradient $\mathbf{F}=-\nabla \Phi$ of a gravitational potential $\Phi$ which is related to the stellar density

$$
\rho(\mathbf{r}, t)=\sum_{i=1}^{N} m \delta\left(\mathbf{r}-\mathbf{r}_{i}\right)
$$

by the Poisson equation

$$
\Delta \Phi=4 \pi G \rho .
$$


Furthermore, the equations of motion (Newton's equations) can be put in the Hamiltonian form

$$
\begin{aligned}
& m \frac{d \mathbf{r}_{i}}{d t}=\frac{\partial H}{\partial \mathbf{v}_{i}}, \quad m \frac{d \mathbf{v}_{i}}{d t}=-\frac{\partial H}{\partial \mathbf{r}_{i}}, \\
& H=\frac{1}{2} \sum_{i=1}^{N} m v_{i}^{2}-\sum_{i<j} \frac{G m^{2}}{\left|\mathbf{r}_{i}-\mathbf{r}_{j}\right|}
\end{aligned}
$$

In the analogy between stellar systems and two-dimensional vortices, the star density $\rho$ plays the role of the vorticity $\omega$, the force $\mathbf{F}$ the role of the velocity $\mathbf{V}$ and the gravitational potential $\Phi$ the role of the streamfunction $\psi$. The crucial point to realize is that, for the two systems, the interaction is a long-range unshielded Coulombian interaction (in $D=3$ or $D=2$ dimensions). This makes the connexion between point vortices and stellar systems deeper than between point vortices and electric charges for example. In particular, point vortices can organize into large scale clusters, like stars in galaxies, while the distribution of electric charges in a neutral plasma is uniform. There are, on the other hand, fundamental differences between stars and vortices. In particular, a star creates an acceleration while a vortex creates a velocity. On the other hand, the gravitational interaction is attractive and directed along the line joining the particles while the interaction between vortices is rotational and perpendicular to the line joining the vortices.

Despite these important differences, the statistical mechanics of $2 \mathrm{D}$ vortices and stellar systems are relatively similar. Like the point vortex gas, the selfgravitating gas is described at statistical equilibrium by the Boltzmann distribution

$$
\langle\rho\rangle=A e^{-\beta \Phi},
$$

obtained by maximizing the Boltzmann entropy at fixed mass $M$ and energy $E$. Its structure is therefore determined by solving the Boltzmann-Poisson equation

$$
\Delta \Phi=4 \pi G A e^{-\beta \Phi},
$$

where $A$ and $\beta>0$ have to be related to $M$ and $E$. This statistical mechanics approach has been developed principally for globular clusters relaxing towards equilibrium via two-body encounters [9]. It is clear that the Boltzmann-Poisson equation (102) is similar to the Boltzmann-Poisson equation (45) for point vortices at negative temperatures. The density profile determined by these equations is a decreasing function of the distance, which corresponds to a situation of clustering (see Figs. 5 and 8 ). The similarity of the maximum entropy problem for stars and vortices, and the Boltzmann-Poisson equations (102) (45), is a first manifestation of the formal analogy existing between these two systems.

However, due to the different dimension of space $(D=3$ for stars instead of $D=2$ for vortices), the mathematical problems differ in the details. First of all, the density profile determined by the Boltzmann-Poisson equation (102) in $D=3$ decreases like $r^{-2}$ at large distances leading to the so-called infinite mass 


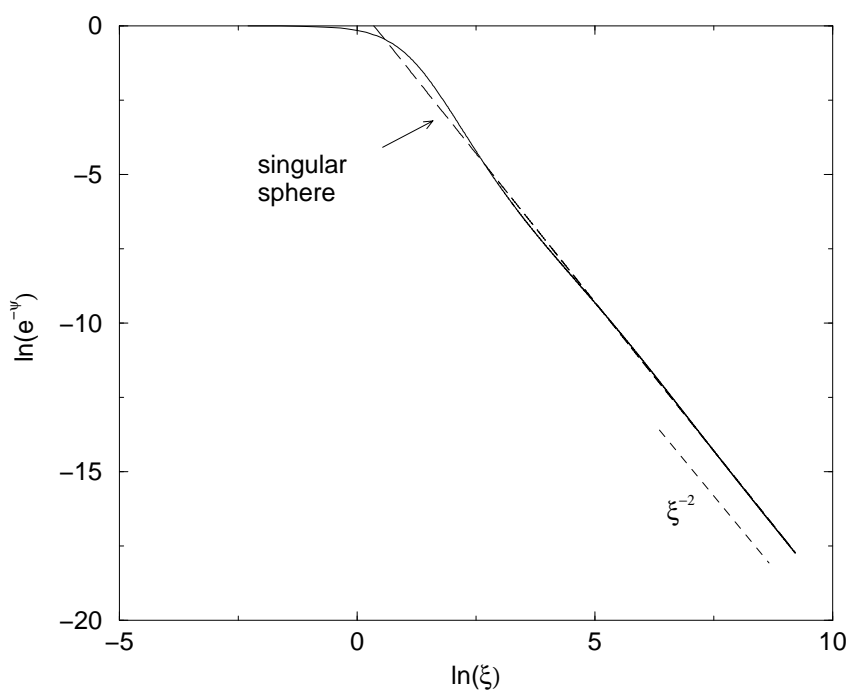

Fig. 8. Density profile of the self-gravitating gas at statistical equilibrium. The dashed line corresponds to the singular solution $\rho=1 / 2 \pi G \beta r^{2}$.

problem since $M=\int_{0}^{+\infty} \rho 4 \pi r^{2} d r \rightarrow+\infty$ 19]. There is no such problem for point vortices in two dimensions: the vorticity decreases like $r^{-4}$, or even more rapidly if the conservation of angular momentum is accounted for, and the total circulation $\Gamma=\int_{0}^{+\infty} \omega 2 \pi r d r$ is finite. The infinite mass problem implies that no statistical equilibrium state exists for open star clusters, even in theory. A system of particles in gravitational interaction tends to evaporate so that the final state is just two stars in Keplerian orbit. This evaporation process has been clearly identified in the case of globular clusters which gradually lose stars to the benefit of a neighboring galaxy. In fact, the evaporation is so slow that we can consider in a first approximation that the system passes by a succession of quasiequilibrium states described by a truncated isothermal distribution function (Michie-King model) [9]. This justifies the statistical mechanics approach in that sense. Another way of solving the infinite mass problem is to confine the system within a box of radius $R$. However, even in that case, the notion of equilibrium poses problem regarding what now happens at the center of the configuration.

The equilibrium phase diagram $(E, T)$ for bounded self-gravitating systems is represented in Fig. 9. The caloric curve has a striking spiral behavior parametrized by the density contrast $\mathcal{R}=\rho(0) / \rho(R)$ going from 1 (homogeneous system) to $+\infty$ (singular sphere) as we proceed along the spiral. There is no equilibrium state below $E_{c}=-0.335 G M^{2} / R$ or $T_{c}=\frac{G M m}{2.52 k R}$ [2, 92$]$. In that case, the system is expected to collapse indefinitely. This is called gravothermal catastrophe in the microcanonical ensemble (fixed $E$ ) and isothermal collapse in the canonical ensemble (fixed $T$ ). Dynamical models show that the collapse is self-similar and develops a finite time singularity [105, 85, 56, 91, 84, 46. However, although the central density goes to $+\infty$, the shrinking of the core is so rapid that the core 


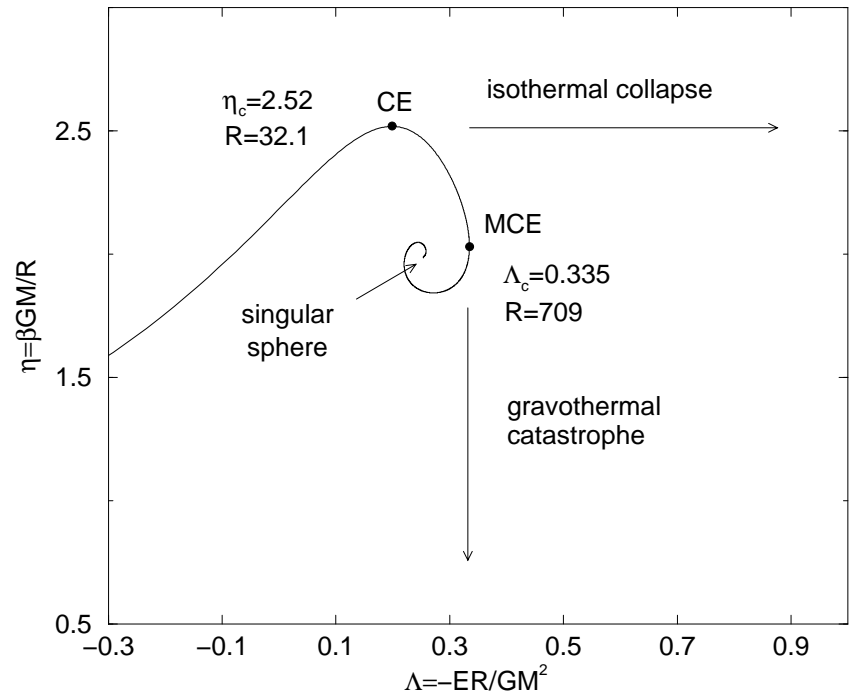

Fig. 9. Equilibrium phase diagram for self-gravitating systems confined within a box. For sufficiently low energy or temperature, there is no equilibrium state and the system undergoes gravitational collapse.

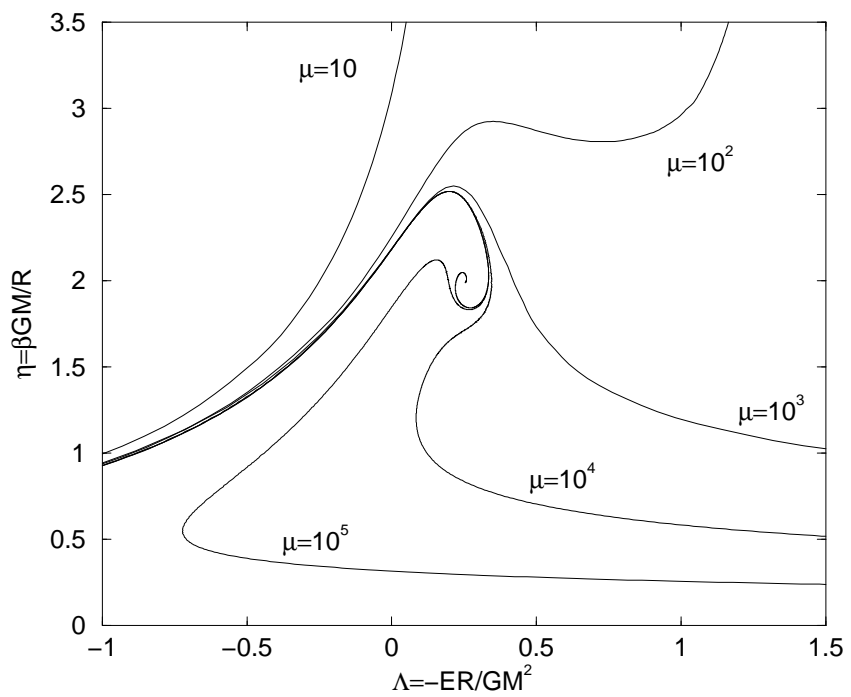

Fig. 10. Equilibrium phase diagram for self-gravitating fermions 41]. The degeneracy parameter $\mu$ plays the role of a small-scale cut-off $\epsilon \sim 1 / \mu$. For $\epsilon \rightarrow 0$, the classical spiral of Fig. 9 is recovered. 
mass goes to zero. Therefore, the singularity contains no mass and this process alone cannot lead to a black hole.

Since the $T(E)$ curve has turning points, this implies that the microcanonical and canonical ensembles are not equivalent and that phase transitions will occur [103]. In the microcanonical ensemble, the series of equilibria becomes unstable after the first turning point of energy $(M C E)$ corresponding to a density contrast of 709. At that point, the solutions pass from local entropy maxima to saddle points. In the canonical ensemble, the series of equilibria becomes unstable after the first turning point of temperature $(C E)$ corresponding to a density contrast of 32.1. At that point, the solutions pass from minima of free energy $(F=E-T S)$ to saddle points. It can be noted that the region of negative specific heats between $(C E)$ and $(M C E)$ is stable in the microcanonical ensemble but unstable in the canonical ensemble, as expected on general physical grounds. The thermodynamical stability of isothermal spheres can be deduced from the topology of the $\beta-E$ curve by using the turning point criterion of Katz [75] who has extended Poincaré's theory of linear series of equilibria. The stability problem can also be reduced to the study of an eigenvalue equation associated with the second order variations of entropy or free energy as studied by Padmanabhan [102] in the microcanonical ensemble and by Chavanis [37] in the canonical ensemble. This study has been recently extended to other statistical ensembles [44]: grand canonical, grand microcanonical, isobaric.... The same stability limits as Katz are obtained but this method provides in addition the form of the density perturbation profiles that trigger the instability at the critical points. It also enables one to show a clear equivalence between thermodynamical stability in the canonical ensemble and dynamical stability with respect to the Navier-Stokes equations (Jeans problem) [37,44. These analytical methods can be extended to general relativity 38. It must be stressed, however, that the statistical equilibrium states of self-gravitating systems are at most metastable: there is no global maximum of entropy or free energy for a classical system of point masses in gravitational interaction [2].

Phase transitions in self-gravitating systems can be studied in detail by introducing a small-scale cut-off $\epsilon$ in order to regularize the potential. This can be achieved for example by considering a system of self-gravitating fermions (in which case an effective repulsion is played by the Pauli exclusion principle) [62, 8, 52, 41, 43 or a hard spheres gas [3, 103, 120]. Other forms of regularization are possible [59]128,45]. For these systems, there can still be gravitational collapse but the core will cease to shrink when it feels the influence of the cut-off. The result is the formation of a compact object with a large mass: a "fermion ball" or a hard spheres "condensate". The equilibrium phase diagram of self-gravitating fermions is represented in Fig. 10 and has been discussed at length by Chavanis 41 in the light of an analytical model. The introduction of a small-scale cutoff has the effect of unwinding the classical spiral of Fig. 9. For a small cut-off $\epsilon \ll 1$, the trace of the spiral is still visible and the $T(E)$ curve is multivalued (Fig. 11). This can lead to a gravitational first order phase transition between a gaseous phase with an almost homogeneous density profile (upper branch) and a 


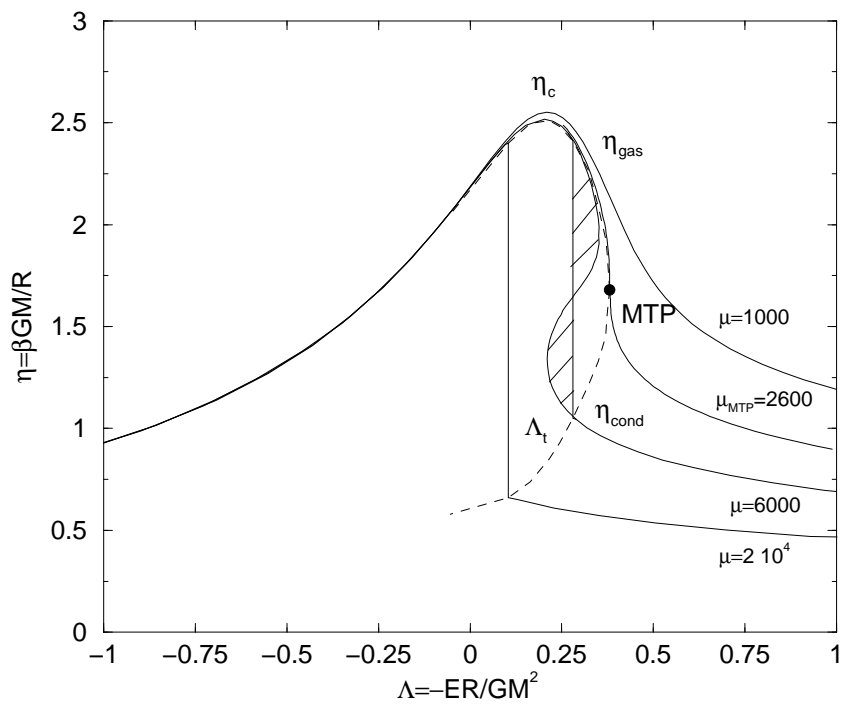

Fig. 11. Enlargement of the phase diagram near the tricritical point in the microcanonical ensemble. A priori, the phase transition should occur at the energy $E_{t}(\mu)$ at which the gaseous phase and the condensed phase have the same entropy. In fact, the entropic barrier played by the unstable solution on the wiggling branch is so hard to cross that the transition will not occur at $E_{t}$ but rather at, or near, $E_{c}$ the point of gravothermal catastrophe $[77,45]$.

condensed phase with a core-halo structure (lower branch). At a critical cut-off value $\epsilon=\epsilon_{M T P}$, the two phases merge and the gravitational phase transition disapears. This particular point is sometimes called a microcanonical tricritical point (MTP).

For $\epsilon>\epsilon_{M T P}$, the curve has the form of Fig. 12. The $T(E)$ curve is now univalued so that the equilibrium states are always stable in the microcanonical ensemble (they are global entropy maxima). In particular, the region of negative specific heats (leading to a convex dip in the entropy vs energy plot [45]) is allowed in the microcanonical ensemble. By contrast, the $E(T)$ curve is multivalued and this can lead to a normal first order phase transition in the canonical ensemble. The gaseous and condensed phases are thus connected by a Maxwell plateau which replaces the region of negative specific heats. At $\epsilon=\epsilon_{C T P}$, the two phases merge, the specific heat becomes infinite and the phase transition is second order. This particular point is sometimes called a canonical tricritical point (CTP). For $\epsilon>\epsilon_{C T P}$, the $T(E)$ curve is monotonic and the specific heat is positive. Therefore, as the cut-off parameter $\epsilon$ increases, the self-gravitating gas consecutively exhibits gravitational first order, normal first order, second-order and no phase transition at all 45. A similar behavior exists for the BlumeEmery-Griffiths (BEG) model with infinite range interaction [6] and is probably representative of other systems presenting inequivalence of ensembles (small systems or systems with long-range interactions). 


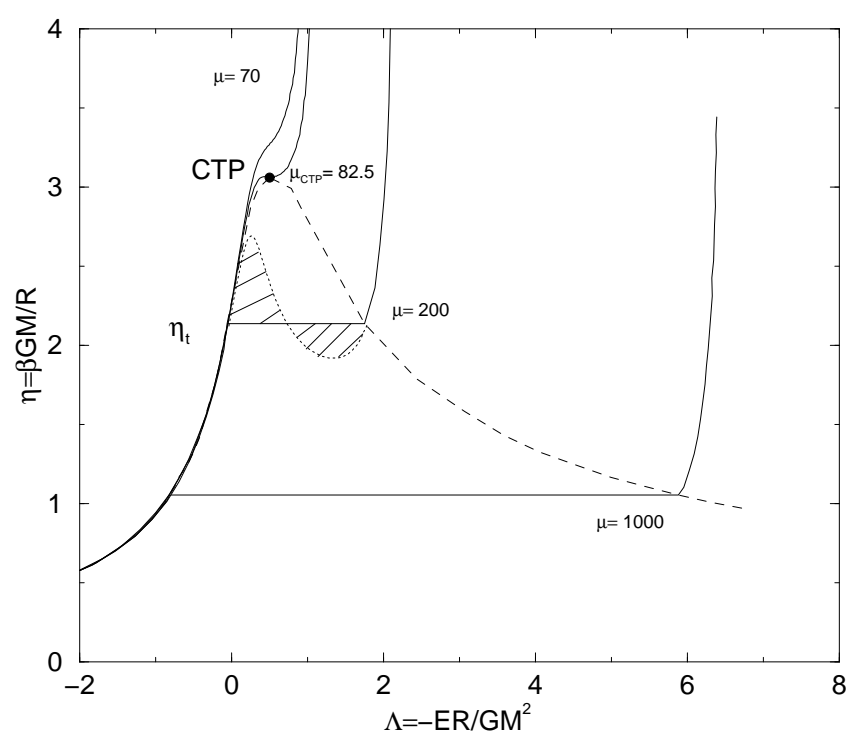

Fig. 12. Same as Fig. 11 near the tricritical point in the canonical ensemble. The region of negative specific heats, allowed in the microcanonical ensemble, is replaced by a phase transition in the canonical ensemble. The notion of metastability is discussed in 41,45 .

The equilibrium phase diagram of point vortices in two dimensions is completely different (Fig. 6). Since there is no turning point of energy or temperature, we can immediately infer that the equilibria are always stable and that the microcanonical and canonical ensembles are equivalent. In the microcanonical ensemble, an equilibrium state exists for all values of energy. Therefore, there is no "gravothermal catastrophe" in two dimensions [76]. The solutions of the Boltzmann-Poisson system for arbitrary dimension $D$ and the disappearance of the spiral as we approach the critical dimension $D=2$ have been studied in 116 (note that the spiral also disapears for $D \geq 10$ ). There exists, on the other hand, a critical inverse temperature $\eta_{c}=-4$ in two dimensions below which there is no equilibrium state in the canonical ensemble. This can lead to a situation of collapse 116 but, as indicated previously, it is not clear whether the canonical ensemble makes sense for a gas of point vortices.

\section{Statistics of velocity fluctuations arising from a random distribution of point vortices}

\subsection{The marginal Gaussian distribution}

The aim of equilibrium statistical mechanics is to predict the final configuration of a system resulting from a complex evolution. We seek now to develop a kinetic theory of point vortices to determine how the system will reach this equilibrium 
configuration. The first problem to consider is the characterization of the velocity fluctuations experienced by a point vortex. These fluctuations are responsible for a diffusion process which is one of the driving source of the evolution. In this section, we describe a simple stochastic model 69, 97, 126, 55, 82, 47, 48, 40] from which we can obtain an estimate of the diffusion coefficient of point vortices [47. We follow the presentation of Chavanis \& Sire 447,48].

Let us consider a collection of $N$ point vortices randomly distributed in a disk of radius $R$. We assume that the vortices have a Poisson distribution, i.e. their positions are independent and uniformly distributed in average over the entire domain. In terms of statistical mechanics, this corresponds to an equilibrium state with $\beta=0$ in a domain with no specific symmetry or $\beta \rightarrow+\infty$ (i.e. a state of minimum energy) if the angular momentum is conserved. We are particularly interested in the "thermodynamic limit" in which the number of vortices and the size of the domain go to infinity $(N \rightarrow \infty, R \rightarrow \infty)$ in such a way that the vortex density $n=\frac{N}{\pi R^{2}}$ remains finite. In this limit, the Poisson distribution is stationary and is well-suited to the analysis of the fluctuations. For simplicity, we assume that the vortices have the same circulation $\gamma$. There is therefore a solid rotation of the system $\langle\mathbf{V}\rangle=\frac{1}{2} n \gamma \mathbf{r}_{\perp}$. We shall work in a rotating frame of reference so as to ignore this solid rotation. The generalization of our results to a spectrum of circulations among the vortices is relatively straightforward 47.

The velocity $\mathbf{V}$ occurring at the center $O$ of the domain is the sum of the velocities $\boldsymbol{\Phi}_{i}(i=1, \ldots, N)$ produced by the $N$ vortices:

$$
\mathbf{V}=\sum_{i=1}^{N} \boldsymbol{\Phi}_{i}, \quad \boldsymbol{\Phi}_{i}=-\frac{\gamma}{2 \pi} \frac{\mathbf{r}_{\perp i}}{r_{i}^{2}},
$$

where $\mathbf{r}_{i}$ denotes the position of the $i^{t h}$ vortex relative to the point under consideration and, by definition, $\mathbf{r}_{\perp i}$ is the vector $\mathbf{r}_{i}$ rotated by $+\pi / 2$. Since the vortices are randomly distributed, the velocity $\mathbf{V}$ fluctuates. It is therefore of interest to study the statistics of these fluctuations, i.e. the probability $W(\mathbf{V}) d^{2} \mathbf{V}$ that $\mathbf{V}$ occurs between $\mathbf{V}$ and $\mathbf{V}+d \mathbf{V}$. Basically, we have to determine the distribution of a sum of random variables. If the variance of the individual velocity $\boldsymbol{\Phi}$ were finite, we could immediately apply the central limit theorem and deduce that the distribution of $\mathbf{V}$ is Gaussian. Alternatively, if the variance of $\mathbf{\Phi}$ were rapidly diverging (i.e., algebraically), the distribution of $\mathbf{V}$ would be a Lévy law with an infinite variance and an algebraic tail. In the present case, the problem is intermediate between these two situations because the variance of the velocity created by a single vortex

$$
\left\langle\Phi^{2}\right\rangle=\int_{|\mathbf{r}|=0}^{R} \tau(\mathbf{r}) \Phi^{2} d^{2} \mathbf{r}=\int_{0}^{R} \frac{1}{\pi R^{2}} \frac{\gamma^{2}}{4 \pi^{2} r^{2}} 2 \pi r d r,
$$

diverges slowly, i.e. logarithmically. For that reason, the distribution of $\mathbf{V}$ will be intermediate between Gaussian and Lévy laws. 
The distribution $W_{N}(\mathbf{V})$ of the velocity can be expressed as

$$
W_{N}(\mathbf{V})=\int \prod_{i=1}^{N} \tau\left(\mathbf{r}_{i}\right) d^{2} \mathbf{r}_{i} \delta\left(\mathbf{V}-\sum_{i=1}^{N} \mathbf{\Phi}_{i}\right),
$$

where $\tau\left(\mathbf{r}_{i}\right) d^{2} \mathbf{r}_{i}$ governs the probability of occurrence of the $i$-th point vortex at position $\mathbf{r}_{i}$. In writing this expression, we have assumed that the vortices are identical and uncorrelated. Now, using a method originally due to Markov, we express the $\delta$-function appearing in Eq. (105) in terms of its Fourier transform

$$
\delta(\mathbf{x})=\frac{1}{(2 \pi)^{2}} \int e^{-i \boldsymbol{\rho} \mathbf{x}} d^{2} \boldsymbol{\rho} .
$$

With this transformation, $W_{N}(\mathbf{V})$ becomes

$$
W_{N}(\mathbf{V})=\frac{1}{4 \pi^{2}} \int A_{N}(\boldsymbol{\rho}) e^{-i \boldsymbol{\rho} \mathbf{V}} d^{2} \boldsymbol{\rho},
$$

with

$$
A_{N}(\boldsymbol{\rho})=\left(\int_{|\mathbf{r}|=0}^{R} e^{i \boldsymbol{\rho} \Phi} \tau(\mathbf{r}) d^{2} \mathbf{r}\right)^{N}
$$

where we have written

$$
\mathbf{\Phi}=-\frac{\gamma}{2 \pi} \frac{\mathbf{r}_{\perp}}{r^{2}} .
$$

If we now suppose that the vortices are uniformly distributed on average, then

$$
\tau(\mathbf{r})=\frac{1}{\pi R^{2}}
$$

and Eq. 108) reduces to

$$
A_{N}(\boldsymbol{\rho})=\left(\frac{1}{\pi R^{2}} \int_{|\mathbf{r}|=0}^{R} e^{i \boldsymbol{\rho} \Phi} d^{2} \mathbf{r}\right)^{N} .
$$

Since

$$
\frac{1}{\pi R^{2}} \int_{|\mathbf{r}|=0}^{R} d^{2} \mathbf{r}=1
$$

we can rewrite our expression for $A_{N}(\boldsymbol{\rho})$ in the form

$$
A_{N}(\boldsymbol{\rho})=\left(1-\frac{1}{\pi R^{2}} \int_{|\mathbf{r}|=0}^{R}\left(1-e^{i \boldsymbol{\rho} \mathbf{\Phi}}\right) d^{2} \mathbf{r}\right)^{N} .
$$

We now consider the limit in which the number of vortices and the size of the domain go to infinity in such a way that the density remains finite:

$$
N \rightarrow \infty, \quad R \rightarrow \infty, \quad n=\frac{N}{\pi R^{2}} \quad \text { finite. }
$$


If the integral occurring in Eq. (113) increases less rapidly than $N$, then

$$
A(\boldsymbol{\rho})=e^{-n C(\boldsymbol{\rho})},
$$

with

$$
C(\boldsymbol{\rho})=\int_{|\mathbf{r}|=0}^{R}\left(1-e^{i \boldsymbol{\rho} \Phi}\right) d^{2} \mathbf{r} .
$$

We have dropped the subscript $N$ to indicate that the limit $N \rightarrow \infty$, in the previous sense, has been taken. Note that $A(\boldsymbol{\rho})$ can still depend on $N$ through logarithmic factors, so that Eq. (114) must be considered as an equivalent of Eq. (108) for large $N$, not a true limit. In fact, it is appropriate to consider that $N \rightarrow+\infty$ but not $\ln N$ since in physical situations the typical number of vortices does not exceed $10^{4}$.

The characteristic function $C(\boldsymbol{\rho})$ can be calculated explicitly by taking $\boldsymbol{\Phi}$ as a variable of integration instead of $\mathbf{r}$ and transforming to polar coordinates 47. The final result remains complicated but the following expression provides a sufficient approximation for our purposes:

$$
C(\boldsymbol{\rho})=\frac{\gamma^{2} \rho^{2}}{16 \pi} \ln \left(\frac{4 \pi N}{n \gamma^{2} \rho^{2}}\right) .
$$

Since $C(\boldsymbol{\rho})$ diverges weakly with $N$ (logarithmically), the limiting process leading to formula (114) is permissible. The velocity distribution $W(\mathbf{V})$ is simply the Fourier transform of $A(\boldsymbol{\rho})$ with the expression (116) for $C(\boldsymbol{\rho})$. This leads to the following distribution for the velocity fluctuations:

$$
\begin{gathered}
W(\mathbf{V})=\frac{4}{n \gamma^{2} \ln N} e^{-\frac{4 \pi}{n \gamma^{2} \ln N} V^{2}} \quad\left(V \ll V_{\text {crit }}(N)\right), \\
W(\mathbf{V})=\frac{n \gamma^{2}}{4 \pi^{2} V^{4}} \quad\left(V \gg V_{\text {crit }}(N)\right), \\
V_{\text {crit }}(N) \sim\left(\frac{n \gamma^{2}}{4 \pi} \ln N\right)^{1 / 2} \ln ^{1 / 2}(\ln N) .
\end{gathered}
$$

For small fluctuations, the core of the distribution is Gaussian as if the central limit theorem were applicable (this is due to the quadratic behavior of $C(\rho)$ ). For sufficiently large values of $V$, the velocity distribution $W(\mathbf{V})$ decays algebraically as for a Lévy law (this is due to the logarithmic term in $C(\rho)$ ). In the mathematical limit $\ln N \rightarrow+\infty$, the algebraic tail is rejected to infinity and the distribution is purely Gaussian. This is consitent with the generalized form of the central limit theorem described by Ibragimov \& Linnik [67] and used by Min et al. 97] and Weiss et al. [126. However, the convergence is so slow with $N$ that the algebraic tail is always visible for point vortex systems $[97,69,126,82]$. This algebraic tail arises because we are on the frontier between Gaussian and Lévy laws (see Fig. 1.1 of Bouchaud \& Georges [11]). Therefore, we proposed to call Eqs. (117)-(119) the Marginal Gaussian distribution [48]. 
Since the distribution $W(\mathbf{V})$ decreases like $V^{-4}$ for $V \rightarrow \infty$, the variance of the velocity diverges logarithmically. Since $V=\gamma / 2 \pi r$, this corresponds to a divergence at small scales $(r \rightarrow 0)$. On the other hand, if we were to extend the Gaussian distribution (117) for all values of $V$, we would conclude that its variance

$$
\left\langle V^{2}\right\rangle=\frac{n \gamma^{2}}{4 \pi} \ln N
$$

diverges logarithmically when $N \rightarrow \infty$. This corresponds to a divergence at large scales $(R \rightarrow+\infty)$. We can recover these results more directly by calculating the variance of $\mathbf{V}$ from Eq. (103). Indeed,

$$
\left\langle V^{2}\right\rangle=N \int_{L_{m i n}}^{R} \frac{1}{\pi R^{2}} \frac{\gamma^{2}}{4 \pi^{2} r^{2}} 2 \pi r d r=\frac{n \gamma^{2}}{2 \pi} \ln \left(\frac{R}{L_{\min }}\right),
$$

where $L_{\text {min }}$ is a lower cut-off and $R$ an upper cut-off played here by the size of the domain.

We can give a physical interpretation of the algebraic tail of the velocity distribution in terms of the nearest neighbor approximation. Let us calculate the velocity distribution due to the nearest neighbor. For that purpose, we must first determine the probability $\tau_{n . n}(r) d r$ that the position of the nearest neighbor occurs between $r$ and $r+d r$. Clearly, $\tau_{n . n}(r) d r$ is equal to the probability that no vortices exist interior to $r$ times the probability that a vortex (any) exists in the annulus between $r$ and $r+d r$. Therefore, it must satisfy an equation of the form

$$
\tau_{n . n}(r) d r=\left(1-\int_{0}^{r} \tau_{n . n}\left(r^{\prime}\right) d r^{\prime}\right) n 2 \pi r d r
$$

where $n=\frac{N}{\pi R^{2}}$ denotes the mean density of vortices in the disk. Differentiating Eq. (122) with respect to $r$ we obtain

$$
\frac{d}{d r}\left[\frac{\tau_{n . n}(r)}{2 \pi n r}\right]=-\tau_{n . n}(r) .
$$

This equation is readily integrated with the condition $\tau_{n . n}(r) \sim 2 \pi n r$ as $r \rightarrow 0$, and we find

$$
\tau_{n . n}(r)=2 \pi n r e^{-\pi n r^{2}} .
$$

This is the distribution of the nearest neighbor in a random distribution of particles. From this formula, we can obtain the exact value for the "average distance" $d$ between vortices. By definition,

$$
d=\int_{0}^{+\infty} \tau_{n . n}(r) r d r
$$

Hence,

$$
d=\frac{1}{2 \sqrt{n}}
$$


If we assume that the velocity $\mathbf{V}$ is entirely due to the nearest neighbor, then

$$
W_{n . n}(\mathbf{V}) d^{2} \mathbf{V}=\tau_{n . n}(\mathbf{r}) d^{2} \mathbf{r},
$$

with

$$
\mathbf{V}=-\frac{\gamma}{2 \pi} \frac{\mathbf{r}_{\perp}}{r^{2}}
$$

Using Eq. (124), we obtain

$$
W_{n . n}(\mathbf{V})=\frac{n \gamma^{2}}{4 \pi^{2} V^{4}} e^{-\frac{n \gamma^{2}}{4 \pi V^{2}}} .
$$

The nearest neighbor approximation is expected to give relevant results only for large values of the velocity. Thus, we can make the additional approximation

$$
W_{n . n}(\mathbf{V})=\frac{n \gamma^{2}}{4 \pi^{2} V^{4}},
$$

in perfect agreement with Eq. (118) valid for $V \gg V_{\text {crit }}$. This shows that the algebraic tail of the velocity distribution is essentially produced by the nearest neighbor as for a Lévy law.

Note also that the typical velocity due to the nearest neighbor is $V_{n . n} \sim$ $\gamma / 2 \pi d$ where $d$ is the inter-vortex distance (126). Hence $V_{n . n}^{2} \sim n \gamma^{2} / \pi^{2}$. Comparing this result with Eq. (120), we see that the velocity due to all the vortices is, up to a logarithmic factor, of the same order as the velocity due to the nearest neighbor. We can say, in some sense, that the velocity fluctuation is marginally dominated by the nearest neighbor and that collective effects are responsible for logarithmic corrections. In fact, we can show [40] that the "effective velocity" created by a vortex at a distance $r$ from the point under consideration is given by

$$
V_{e f f}=\frac{\gamma}{2 \pi r} \frac{1}{1+r / \Lambda},
$$

where $\Lambda \sim d$ is of the order of the inter-vortex distance. For $r \gg d$, the "effective" velocity decays as $r^{-2}$ instead of the ordinary $r^{-1}$ law recovered for $r \ll d$. This result indicates that, in a statistical sense, the velocity produced by a vortex is shielded by "cooperative" effects.

\subsection{The speed of fluctuations}

The function $W(\mathbf{V})$ does not provide us with all the necessary information concerning the fluctuations of $\mathbf{V}$. An important aspect of the problem concerns the speed of fluctuations, i.e. the typical duration $T(V)$ of the velocity fluctuation $\mathbf{V}$. This requires the knowledge of the bivariate probability $W(\mathbf{V}, \mathbf{A}) d^{2} \mathbf{V} d^{2} \mathbf{A}$ to measure simultaneously a velocity $\mathbf{V}$ with a rate of change

$$
\mathbf{A}=\frac{d \mathbf{V}}{d t}=\sum_{i=1}^{N} \boldsymbol{\psi}_{i}
$$




$$
\boldsymbol{\psi}_{i}=-\frac{\gamma}{2 \pi}\left(\frac{\mathbf{v}_{\perp i}}{r_{i}^{2}}-\frac{2\left(\mathbf{r}_{i} \mathbf{v}_{i}\right) \mathbf{r}_{\perp i}}{r_{i}^{4}}\right),
$$

where $\mathbf{v}_{i}=d \mathbf{r}_{i} / d t$ is the velocity of vortex $i$. Then, the duration $T(V)$ can be estimated by the formula

$$
T(V)=\frac{|\mathbf{V}|}{\sqrt{\left\langle A^{2}\right\rangle_{\mathbf{V}}}}
$$

where

$$
\left\langle A^{2}\right\rangle_{\mathbf{V}}=\frac{\int W(\mathbf{V}, \mathbf{A}) A^{2} d^{2} \mathbf{A}}{W(\mathbf{V})},
$$

is the mean square acceleration associated with a velocity fluctuation $\mathbf{V}$.

To determine the speed of fluctuations, we need therefore to calculate the bivariate probability $W_{N}(\mathbf{V}, \mathbf{A})$ to measure simultaneously a velocity $\mathbf{V}$ with a rate of change $\mathbf{A}=d \mathbf{V} / d t$. According to Eqs. (103), (132) and (133), $\mathbf{V}$ and A are the sum of $N$ random variables $\boldsymbol{\Phi}_{i}$ and $\boldsymbol{\psi}_{i}$ depending on the positions $\mathbf{r}_{i}$ and on the velocities $\mathbf{v}_{i}$ of the point vortices. Unlike material particles, the variables $\left\{\mathbf{r}_{i}, \mathbf{v}_{i}\right\}$, for different $i$ 's, are not independent because the velocities of the vortices are determined by the configuration $\left\{\mathbf{r}_{i}\right\}$ of the system as a whole (see Eq. (103)). To be able to solve the problem analytically, we shall make a decorrelation approximation and treat $\left\{\mathbf{r}_{i}, \mathbf{v}_{i}\right\}(i=1, \ldots, N)$ as independent variables governed by the distribution

$$
\tau(\mathbf{r}, \mathbf{v})=\frac{1}{\pi R^{2}} \times \frac{4}{n \gamma^{2} \ln N} e^{-\frac{4 \pi}{n \gamma^{2} \ln N} v^{2}},
$$

resulting from Eqs. (110) and (117). It is remarkable that the distribution (136) is formally equivalent to the Maxwell-Boltzmann statistics of material particles at equilibrium. Owing to this analogy, we can interpret the variance

$$
\overline{v^{2}}=\frac{n \gamma^{2}}{4 \pi} \ln N
$$

as a kind of kinetic "temperature" of the point vortices. It should not be confused with the temperature $\beta$ introduced in Sec. 2 and measuring the clustering of the vortices (in the present case, $\beta=0$ since the vortices are uniformly distributed in average).

When this decorrelation hypothesis is implemented, a straightforward generalization of the method used in Sec. 3.1 yields

$$
W_{N}(\mathbf{V}, \mathbf{A})=\frac{1}{16 \pi^{4}} \int A_{N}(\boldsymbol{\rho}, \boldsymbol{\sigma}) e^{-i(\boldsymbol{\rho} \mathbf{V}+\boldsymbol{\sigma} \mathbf{A})} d^{2} \boldsymbol{\rho} d^{2} \boldsymbol{\sigma}
$$

with

$$
A_{N}(\boldsymbol{\rho}, \boldsymbol{\sigma})=\left(\int_{|\mathbf{r}|=0}^{R} \int_{|\mathbf{v}|=0}^{+\infty} \tau(\mathbf{r}, \mathbf{v}) e^{i(\boldsymbol{\rho} \boldsymbol{\Phi}+\boldsymbol{\sigma} \psi)} d^{2} \mathbf{r} d^{2} \mathbf{v}\right)^{N}
$$

where we have defined

$$
\boldsymbol{\Phi}=-\frac{\gamma}{2 \pi} \frac{\mathbf{r}_{\perp}}{r^{2}}
$$




$$
\boldsymbol{\psi}=-\frac{\gamma}{2 \pi}\left(\frac{\mathbf{v}_{\perp}}{r^{2}}-\frac{2(\mathbf{r v}) \mathbf{r}_{\perp}}{r^{4}}\right) .
$$

Using an integration by parts, the conditional moment of the acceleration for a given velocity fluctuation $\mathbf{V}$ can be expressed as 47 :

$$
W(\mathbf{V})\left\langle A^{2}\right\rangle_{\mathbf{V}}=-\frac{1}{\pi^{2}} \int \frac{\partial A}{\partial\left(\sigma^{2}\right)}(\boldsymbol{\rho}, \mathbf{0}) e^{-i \boldsymbol{\rho} \mathbf{V}} d^{2} \boldsymbol{\rho} .
$$

We therefore need to Taylor expand the function $A(\boldsymbol{\rho}, \boldsymbol{\sigma})$ for $|\boldsymbol{\sigma}| \rightarrow 0$ and carry out the integration in Eq. (142). The calculation is relatively tricky but can be done analytically [47]. Substituting the resulting expression for $\left\langle A^{2}\right\rangle_{\mathbf{V}}$ in Eq. (134), we obtain the following estimate for the speed of fluctuations:

$$
\begin{gathered}
T(V)=\frac{4 \sqrt{\pi} V}{n^{3 / 2} \gamma^{2} \ln N} e^{-\frac{2 \pi}{n \gamma^{2} \ln N} V^{2}} \quad\left(V \ll V_{\text {crit }}(N)\right), \\
T(V)=\frac{1}{\sqrt{\pi n \ln N}} \frac{1}{V} \quad\left(V \gg V_{\text {crit }}(N)\right) .
\end{gathered}
$$

For weak and large fluctuations, $T(V)$ decreases to zero like $V$ and $V^{-1}$ respectively. These asymptotic behaviors have a clear physical meaning in the nearest neighbor approximation. When $r=\gamma / 2 \pi V$ is small, corresponding to large velocities, it is highly improbable that another vortex will enter the disk of radius $r$ before long. By contrast, on a short time scale $T \sim r / \bar{v} \sim(\gamma / \bar{v}) V^{-1}$, the vortex will have left the disk. When $r=\gamma / 2 \pi V$ is large, corresponding to small velocities, the probability that the vortex will remain alone in the disk is low. The characteristic time before another vortex enters the disk varies like the inverse of the number of vortices expected to be present in the disk, i.e. $T \sim(r / \bar{v}) 1 / n \pi r^{2} \sim(1 / n \gamma \bar{v}) V$. The demarcation between weak and strong fluctuations corresponds to $V \sim \gamma n^{1 / 2}$, i.e. to the velocity produced by a vortex distant $n^{-1 / 2}$ from the point under consideration. These asymptotic behaviors are also consistent with the theory of Smoluchowski concerning the persistence of fluctuations (see discussion in [47).

The average duration of a velocity fluctuation is defined by

$$
\langle T\rangle=\int_{0}^{+\infty} T(V) W(V) 2 \pi V d V .
$$

To leading order in $\ln N$, we obtain

$$
\langle T\rangle=\frac{4}{3}\left(\frac{\pi}{6}\right)^{1 / 2} \frac{1}{n \gamma \sqrt{\ln N}} .
$$

This formula shows that the typical duration of a velocity fluctuation scales like

$$
T_{t y p} \sim \frac{1}{n \gamma \sqrt{\ln N}}
$$

This corresponds to the typical time needed by a vortex moving with a typical velocity $\left\langle V^{2}\right\rangle^{1 / 2}$ (given by Eq. (120)) to cross the interparticle distance $d \sim$ $n^{-1 / 2}$, as expected from general physical grounds 126 . 


\subsection{The diffusion coefficient}

According to the previous discussion, we can characterize the fluctuations of the velocity of a point vortex by two functions: a function $W(\mathbf{V})$ which governs the occurrence of the velocity $\mathbf{V}$ and a function $T(V)$ which determines the typical time during which the vortex moves with this velocity. Since the velocity fluctuates on a typical time $T_{t y p}=d / \sqrt{\left\langle V^{2}\right\rangle}$ which is much smaller than the dynamical time $t_{D}=R / \sqrt{\left\langle V^{2}\right\rangle}$ needed by the vortex to cross the entire domain, the motion of the vortex will be essentially stochastic. If we denote by $P(\mathbf{r}, t)$ the probability density that the particle be found in $\mathbf{r}$ at time $t$, then $P(\mathbf{r}, t)$ will satisfy the diffusion equation

$$
\frac{\partial P}{\partial t}=D \Delta P
$$

If the particle is at $\mathbf{r}=\mathbf{r}_{0}$ at time $t=0$, the solution of Eq. (148) is clearly

$$
P\left(\mathbf{r}, t \mid \mathbf{r}_{0}\right)=\frac{1}{4 \pi D t} e^{-\frac{\left|\mathbf{r}-\mathbf{r}_{0}\right|^{2}}{4 D t}},
$$

where $D$ is the diffusion coefficient. The mean square displacement that the particle is expected to suffer during an interval of time $\Delta t$ large with respect to the fluctuation time $T_{t y p}$, is

$$
\left\langle(\Delta \mathbf{r})^{2}\right\rangle=4 D \Delta t
$$

We can obtain another expression for $\left\langle(\Delta \mathbf{r})^{2}\right\rangle$ in terms of the functions $W(\mathbf{V})$ and $T(V)$ defined in the previous sections. Indeed, dividing the interval

$$
\Delta \mathbf{r}=\int_{t}^{t+\Delta t} \mathbf{V}\left(t^{\prime}\right) d t^{\prime}
$$

into a succession of discrete increments in position with amount $T\left(V_{i}\right) \mathbf{V}_{i}$, we readily establish that

$$
\left\langle(\Delta \mathbf{r})^{2}\right\rangle=\left\langle T(V) V^{2}\right\rangle \Delta t
$$

Combining Eqs. (150) and (152) we obtain an alternative expression for the diffusion coefficient in the form

$$
D=\frac{1}{4} \int T(V) W(\mathbf{V}) V^{2} d^{2} \mathbf{V}
$$

Substituting for $T(V)$ and $W(\mathbf{V})$ in the foregoing expression, we obtain to leading order in $\ln N$, 47 ,

$$
D=\frac{1}{72}\left(\frac{6}{\pi}\right)^{1 / 2} \gamma \sqrt{\ln N}
$$

We should not give too much credit to the numerical factor appearing in Eq. (154) since the definition (134) of $T(V)$ is just an order of magnitude. Note that the scaling form of $D$ is consistent with the expression

$$
D \sim T_{\text {typ }}\left\langle V^{2}\right\rangle \sim \gamma \sqrt{\ln N}
$$

that one would expect on general physical grounds. 


\subsection{Application to 2D decaying turbulence}

The previous results have some direct applications to the context of 2D decaying turbulence. The relaxation of $2 \mathrm{D}$ decaying turbulence is a three-stage process: during an initial transient period, the fluid organizes itself from random fluctuations and a population of coherent vortices emerges. Then, when two like-sign vortices come into contact they merge and form a bigger structure. As time goes on, the vortex number decreases and their average size increases, in a process reminiscent of a coarsening stage. Finally, when only one dipole is left, it decays diffusively due to inherent viscosity. Direct numerical simulations [18] and experiments [61] show that the typical core vorticity $\omega$ remains constant during the course of the evolution and that the density decreases like $n \sim t^{-\xi}$ with $\xi \approx 0.7$. As the energy $E \sim \int \mathbf{u}^{2} d^{2} \mathbf{r} \sim n \omega^{2} a^{4} \sim n a^{4}$ is conserved throughout the evolution, the typical vortex radius increases like $a \sim t^{\xi / 4}$.

In the punctuated Hamiltonian model 125, the flow is approximated by a collection of vortices with constant vorticity $\pm \omega$ (and circulation $\gamma \sim \pm \omega a_{i}^{2}$ ) whose centers follow the Hamiltonian dynamics (15) (16). When two like-sign vortices with radii $a_{1}$ and $a_{2}$ enter in collision, the resulting vortex keeps the same vorticity and takes a radius $a$ such that $a^{4}=a_{1}^{4}+a_{2}^{4}$ which ensures the conservation of energy (as discussed above). This effective model reproduces the results of the numerical simulations and experiments, with again $\xi \simeq 0.7$. Since the average distance between vortices, of order $d \sim t^{\xi / 2}$, increases more rapidly than their size, the point vortex model should provide increasing accuracy. We emphasize, however, that in the above mentioned studies the density only changes by a factor of order $4 \sim 5$ between the initial time and the final time so that the scaling exponent is measured on less than one decade.

The punctuated Hamiltonian model has been re-investigated recently by Sire \& Chavanis [115] using a renormalization group procedure which allows for much longer time simulations that could otherwise be achieved. It is found that the scaling regime is achieved for very late times and is characterized by a decay exponent $\xi=1$ (an effective exponent $\xi \simeq 0.7$ is recovered for shorter times). In addition, the decay of the total area occupied by the vortices results in a physical process by which merging occurs principally via 3 -vortex collisions involving vortices of different sign. A simple kinetic theory based on an effective 3 -vortex interaction returns this value $\xi=1$. These theoretical results tend to be confirmed by recent direct numerical simulations [86].

During the decay, the vortices diffuse with a coefficient $D \sim \gamma$ given by Eq. (154), where $\gamma \sim \omega a^{2}$ is their circulation (we ignore here logarithmic corrections). If the diffusion coefficient were constant, then the dispersion of the vortices

$$
\left\langle r^{2}\right\rangle \sim D t
$$

would increase linearly with time as in ordinary Brownian motion. However, since $D$ varies with time according to

$$
D \sim \omega a^{2} \sim t^{\xi / 2}
$$


we expect anomalous diffusion, i.e.

$$
\left\langle r^{2}\right\rangle \sim t^{\nu}
$$

with $\nu \neq 1$. Substituting Eq. (157) in Eq. (156), we obtain the following relation between $\nu$ and $\xi$ :

$$
\nu=1+\frac{\xi}{2} .
$$

This formula is in perfect agreement with long time numerical simulations $(\nu=$ $3 / 2$ for $\xi=1)$ [115 and experiments $\left(\nu_{\text {exp }} \sim 1.3-1.4\right.$ in the regime where $\xi=0.7$ ) [61]. This hyperdiffusive behavior can be interpreted in terms of Lévy flights 115, with a large time flight distribution $P(\tau) \sim \tau^{-\mu}$ with $\mu=3-\xi / 2$ in agreement with experiments $\left(\mu_{\text {exp }} \sim 2.6 \pm 0.2\right.$ for $\left.\xi=0.7\right) 61$.

\subsection{The spatial correlations in the velocities arising from a random distribution of point vortices}

The stochastic approach described in Sec. 3.1 can be generalized to obtain exact results concerning the spatial correlations of the velocity fluctuations [48]. First of all, the distribution of the velocity increments between two neighboring points is given by the 2D Cauchy law $69,97,48$ :

$$
W(\delta \mathbf{V})=\frac{2}{\pi n^{2} \gamma^{2}|\delta \mathbf{r}|^{2}}\left(1+\frac{4|\delta \mathbf{V}|^{2}}{n^{2} \gamma^{2}|\delta \mathbf{r}|^{2}}\right)^{-3 / 2}
$$

which is a particular Lévy law. It is also possible to determine an analytical expression for the conditional moment $\langle\delta \mathbf{V}\rangle_{\mathbf{V}}$. We find:

$$
\begin{gathered}
\langle\delta \mathbf{V}\rangle_{\mathbf{V}}=-\frac{n \gamma}{2} B\left(\frac{4 \pi V^{2}}{n \gamma^{2} \ln N}\right)\left\{\delta \mathbf{r}_{\perp}+2 \frac{\left(\mathbf{V}_{\perp} \delta \mathbf{r}\right)}{V^{2}} \mathbf{V}\right\} \quad\left(V \ll V_{c r i t}(N)\right), \\
\langle\delta \mathbf{V}\rangle_{\mathbf{V}}=-\frac{2 \pi}{\gamma} V^{2}\left\{\delta \mathbf{r}_{\perp}+2 \frac{\left(\mathbf{V}_{\perp} \delta \mathbf{r}\right)}{V^{2}} \mathbf{V}\right\} \quad\left(V \gg V_{\text {crit }}(N)\right),
\end{gathered}
$$

where $B(x)$ denotes the function

$$
B(x)=\frac{1}{x}\left(e^{x}-1-x\right) .
$$

On the other hand, the spatial auto-correlation function of the velocity between two points separated by an arbitrary distance is given by 48:

$$
\left\langle\mathbf{V}_{0} \mathbf{V}_{1}\right\rangle=\frac{n \gamma^{2}}{2 \pi} \ln \left(\frac{R}{r_{1}}\right)
$$

This leads to an energy spectrum

$$
E(k)=\frac{n \gamma^{2}}{4 \pi k}\left(1-J_{0}(k R)\right),
$$


which reduces to Novikov's result $E(k)=n \gamma^{2} / 4 \pi k$ for $k \rightarrow+\infty 100$. We observe that the velocity correlation function (164) diverges logarithmically as $r_{1} \rightarrow 0$. This is consistent with the divergence of the variance of the velocity distribution (117)-(119). Therefore, it is more proper to characterize the spatial correlations of the velocity by the function $\left\langle V_{1 \|}\right\rangle=\left\langle\mathbf{V}_{0} \mathbf{V}_{1} /\left|\mathbf{V}_{0}\right|\right\rangle$ which remains finite as $r_{1} \rightarrow 0$. Its evaluation is more complex and leads to the result

$$
\left\langle V_{1 \|}\right\rangle=\left\langle\left|\mathbf{V}_{0}\right|\right\rangle-\left(\frac{n \gamma^{2}}{\pi \ln N}\right)^{1 / 2} \int_{0}^{+\infty} \frac{J_{1}(z)}{z^{2}} \Gamma_{s^{2} z^{2}}\left(\frac{1}{2}\right) d z
$$

where

$$
\Gamma_{x}(p+1)=\int_{0}^{x} e^{-t} t^{p} d t
$$

is the incomplete $\Gamma$-function and

$$
\left\langle\left|\mathbf{V}_{0}\right|\right\rangle=\left(\frac{n \gamma^{2}}{16} \ln N\right)^{1 / 2}, \quad s=\left(\frac{\pi n \ln N}{4}\right)^{1 / 2} r_{1} .
$$

Similarly, the correlation function $K\left(r_{1}\right)=\left\langle\mathbf{V}_{0} \mathbf{V}_{1} / V_{0}^{2}\right\rangle$ is given by

$$
K(s)=1-\frac{4 s^{2}}{\ln N}\left(1-e^{-1 / 4 s^{2}}+\frac{1}{4 s^{2}} E_{1}\left(\frac{1}{4 s^{2}}\right)\right),
$$

where $E_{1}(z)$ denotes the exponential integral

$$
E_{1}(z)=\int_{z}^{+\infty} \frac{e^{-t}}{t} d t
$$

Other results concerning the characterization of the spatial velocity correlations can be found in 48$]$.

\subsection{Statistics of fluctuations of the gravitational field}

The stochastic approach developed in the preceding sections was inspired by the famous work of Chandrasekhar \& von Neumann [20,27,28,24,25] concerning the fluctuations of the gravitational field created by a random distribution of stars. Let us consider a collection of $N$ stars with mass $m$ randomly distributed in a sphere of radius $R$ with a uniform density $n$ in average. The force by unit of mass created at the center $O$ of the domain is

$$
\mathbf{F}=\sum_{i=1}^{N} \boldsymbol{\Phi}_{i}, \quad \boldsymbol{\Phi}_{i}=-\frac{G m}{r_{i}^{3}} \mathbf{r}_{i} .
$$

As before, the problem consists in determining the distribution of a sum of random variables. In the present case, the variance of the force created by one star diverges algebraically

$$
\left\langle\Phi^{2}\right\rangle \sim \frac{3}{4 \pi R^{3}} \int\left(\frac{G m}{r^{2}}\right)^{2} 4 \pi r^{2} d r \sim \frac{1}{r},
$$


so that the distribution of the total force is a Lévy law. This particular Lévy law is known as the Holtsmark distribution since it was first determined by Holtsmark in the context of the electric field created by a gas of simple ions 64. In the thermodynamic limit $N, R \rightarrow+\infty$ with $n=\frac{3 N}{4 \pi R^{3}}$ finite, the distribution of $\mathbf{F}$ can be expressed by the Fourier transform

$$
W(\mathbf{F})=\frac{1}{2 \pi^{2} F} \int_{0}^{+\infty} k \sin (k F) e^{-a k^{3 / 2}} d k,
$$

with

$$
a=\frac{4}{15}(2 \pi G)^{3 / 2} \mathrm{~nm}^{1 / 2}
$$

It has the asymptotic behavior

$$
W(\mathbf{F}) \sim \frac{1}{2} G^{3 / 2} m^{1 / 2} n F^{-9 / 2} \quad(F \rightarrow+\infty)
$$

which can be shown to coincide with the distribution due to the nearest neighbor. The typical force due to the nearest neighbor $F_{n . n} \sim G m / d^{2} \sim G m n^{2 / 3}$ is precisely of the same order as the average value of the force due to all the stars

$$
\langle F\rangle=4 \Gamma\left(\frac{1}{3}\right)\left(\frac{8 \sqrt{2}}{15}\right)^{2 / 3} G m n^{2 / 3},
$$

determined from Eq. (173). This shows that only stars close to the star under consideration determine the fluctuations of the gravitational field. In fact, it is possible to show that the "effective" force created by a star at distance $r$ from the star under consideration is [1]:

$$
F_{e f f}=\frac{G m}{r^{2}} \frac{1}{1+r^{2} / \Lambda^{2}},
$$

where $\Lambda \sim d$ is of the order of the interparticle distance. For weak separations, one has $F_{\text {eff }} \rightarrow G m / r^{2}$ but for large separations $r \gg d$, the effect of individual stars compensate each other and the resulting force is reduced by a factor $(r / d)^{2}$. This corresponds to a shielding of the interaction, in a statistical sense, due to collective effects.

Chandrasekhar \& von Neumann have used this stochastic model to determine the speed of fluctuations $T(F)$ [27, the diffusion coefficient of stars (a calculation completed by Kandrup [72]) and the spatial [28,24] and temporal [25] correlations of the gravitational field etc... There is a complete parallel with the results obtained by Chavanis \& Sire 47,48,40] for point vortices and this is another manifestation of the deep formal analogy between the two systems.

\section{Relaxation of a point vortex in a thermal bath}

\subsection{Analogy with Brownian motion}

We would like now to develop a kinetic theory of point vortices in order to describe their relaxation towards equilibrium. It has to be noted that an equilibrium state can be achieved in very different ways so that the kinetic theory of 
vortices is not unique and depends on the situation contemplated (see Sec. 6). In order to develop an intuition on the problem, we first propose a naive kinetic theory based on an analogy with Brownian theory [30,36]. The starting point of this analogy is to realize that the velocity of a point vortex can be decomposed in two terms: a smoothly varying function of position and time $\langle\mathbf{V}\rangle(\mathbf{r}, t)$ and a function $\boldsymbol{V}(t)$ taking into account the "granularity" of the system. The total velocity of a point vortex can therefore be written:

$$
\mathbf{V}=\langle\mathbf{V}\rangle(\mathbf{r}, t)+\mathcal{V}(t)
$$

The velocity $\langle\mathbf{V}\rangle(\mathbf{r}, t)$ reflects the influence of the system as a whole and is generated by the mean vorticity $\langle\omega\rangle(\mathbf{r}, t)$ according to the Biot \& Savart formula

$$
\langle\mathbf{V}\rangle(\mathbf{r}, t)=-\frac{1}{2 \pi} \mathbf{z} \times \int \frac{\mathbf{r}^{\prime}-\mathbf{r}}{\left|\mathbf{r}^{\prime}-\mathbf{r}\right|^{2}}\langle\omega\rangle\left(\mathbf{r}^{\prime}, t\right) d^{2} \mathbf{r}^{\prime} .
$$

The fluctuation $\boldsymbol{V}(t)$ arises from the difference between the exact distribution $\omega_{\text {exact }}(\mathbf{r}, t)$ of point vortices given by Eq. (13) and their "smoothed-out" distribution $\langle\omega\rangle(\mathbf{r}, t)=n \gamma$. Indeed, if we consider a surface element $\sigma$, the number of point vortices actually present in this area will fluctuate around the mean number $n \sigma$. These fluctuations will be governed by a Poisson distribution with variance $n \sigma$. On account of these fluctuations, the velocity of a vortex will depart from its mean field value $\langle\mathbf{V}\rangle$. The velocity fluctuation $\mathcal{V}$, of order $\gamma / d$ (where $d \sim n^{-1 / 2}$ is the inter-vortex distance), is much smaller than the average velocity $\langle V\rangle$, of order $n \gamma R$ (where $R$ is the domain size), but this term has a cumulative effect which gives rise to a process of diffusion. It makes sense therefore to introduce a stochastic description of the vortex motion such as that for colloidal suspensions in a liquid 81.22 or stars in globular clusters 26]. However, contrary to the ideal Brownian motion, point vortex systems have relatively long correlation times so that $\mathcal{V}(t)$ is not a white noise. This makes the study much more complicated than usual and the technical developments of Sec. 5 are required. However, in order to gain some physical insights into the problem, we shall ignore this difficulty for the moment and describe the system by traditional stochastic processes.

According to Eq. (178), we would naively expect that the evolution of the density probability $P(\mathbf{r}, t)$ would be governed by a diffusion equation of the form

$$
\frac{\partial P}{\partial t}+\langle\mathbf{V}\rangle \nabla P=D \Delta P,
$$

where $D$ is the diffusion coefficient. This would in fact be the case for a passive particle having no retroaction on the vortices or when the distribution of vortices is uniform in average like in Sec. 3. However, this diffusion equation cannot be valid when the system is inhomogeneous. Indeed, Eq. (180) does not converge towards the Boltzmann distribution (44) when $t \rightarrow+\infty$. It seems therefore that a term is missing to act against the diffusion.

This problem is similar to the one encountered by Chandrasekhar in his stochastic approach of stellar dynamics [23]. Chandrasekhar solved the problem by introducing a dynamical friction in order to compensate for the effect 
of diffusion. The occurence of this frictional force is a manifestation of the "fluctuation-dissipation" theorem in statistical mechanics. In the present context, the dynamical friction is replaced by a systematic drift of the vortices [30]. This drift appears when the distribution of vorticity is inhomogeneous. It can be understood in terms of a polarization process like in a phenomenon of induction. In Sec. 1.3, we shall derive the drift term directly from the Liouville equation by using a linear response theory. For the moment, we introduce this term by hands and rewrite the decomposition (178) in the form

$$
\mathbf{V}=\langle\mathbf{V}\rangle-\xi \nabla \psi+\mathcal{V}(t)
$$

where $\xi$ is the drift coefficient. Equation (181) must be viewed as a stochastic equation analogous to the Langevin equation in the ordinary Brownian theory. The corresponding Fokker-Planck equation can be written [30]:

$$
\frac{\partial P}{\partial t}+\langle\mathbf{V}\rangle \nabla P=\nabla(D \nabla P+\xi P \nabla \psi)
$$

The physical interpretation of each term is straightforward. The left hand side (which can be written $d P / d t$ ) is an advection term due to the smooth mean field velocity $\langle\mathbf{V}\rangle$. The right hand side can be written as the divergence of a current $-\nabla \mathbf{J}$ and is the sum of two terms: the first term is a diffusion due to the erratic motion of the vortices caused by the fluctuations of the velocity (see Sec. 3) and the second term accounts for the systematic drift of the vortices due to the inhomogeneity of the vortex cloud. At equilibrium, the drift precisely balances random scatterings and the Boltzmann distribution (44) is settled. More precisely, the condition that the Boltzmann distribution satisfies Eq. (182) identically requires that $D$ and $\xi$ be related according to the relation

$$
\xi=D \beta \gamma
$$

which is a generalization of the Einstein formula to the case of point vortices. It is remarkable that we can obtain such a general relation without, at any point, being required to analyze the mechanism of "collisions". A more rigorous justification of this relation will be given in the next sections in which the diffusion coefficient and the drift term are calculated explicitly. Note that the diffusion current can be written $\mathbf{J}=\chi \nabla \alpha$ where $\alpha=\ln \langle\omega\rangle+\beta \gamma \psi$ is a "generalized potential" which is uniform at equilibrium, see Eq. (44). Therefore, the FokkerPlanck equation (182) is consistent with the linear thermodynamics of Onsager which relates the diffusion currents to the gradients of generalized potentials. The Fokker-Planck equation (182) can also be obtained from a variational formulation (the so-called Maximum Entropy Production Principle): it can be shown to

maximize the rate of entropy production $\dot{S}$ under the constraints brought by the dynamics [36].

\subsection{Diffusion coefficient of point vortices in an inhomogeneous medium}

In this section, we determine the value of the diffusion coefficient $D$ which enters in the Fokker-Planck equation (182). The essential difference with the calculation 
of Sec. 3 is that the vorticity distribution is now inhomogeneous so that there exists a shear in the system. This shear considerably modifies the expression of the diffusion coefficient [30,36]. An explicit expression for $D$ can be obtained in a "thermal bath approximation" which is valid if we are sufficiently close to equilibrium. To be specific, we consider a system of $N$ point vortices at statistical equilibrium with an inverse temperature $\beta_{e q}$. These "field vortices" form the thermal bath. We now introduce a "test vortex" in the system an study its diffusion in the sea of "field vortices". For simplicity, we restrict ourselves to simple equilibrium flows which are either unidirectional or axisymmetric.

In the case of unidirectional flows in the $x$-direction, we define the diffusion coefficient in the $y$ direction by

$$
D=\lim _{t \rightarrow+\infty} \frac{\left\langle(\Delta y)^{2}\right\rangle}{2 t} \quad \text { with } \quad \Delta y=\int_{0}^{t} V_{y}\left(t^{\prime}\right) d t^{\prime},
$$

where $\mathbf{V}(t)$ is given by Eq. (14). It is possible to put the diffusion coefficient in the form of a Kubo formula [36]:

$$
D=\int_{0}^{+\infty}\left\langle V_{y}(t) V_{y}(t-\tau)\right\rangle d \tau
$$

where the quantity in bracket is the velocity auto-correlation function at different times. Using Eq. (14), we have explicitly

$$
D=N \int_{0}^{+\infty} d \tau \int d^{2} \mathbf{r}_{1} V_{y}(1 \rightarrow 0, t) V_{y}(1 \rightarrow 0, t-\tau) P_{e q}\left(y_{1}\right) .
$$

For axisymmetric equilibrium flows, one has similarly

$$
D=\lim _{t \rightarrow+\infty} \frac{\left\langle(\Delta r)^{2}\right\rangle}{2 t} \quad \text { with } \quad \Delta r=\int_{0}^{t} V_{r}\left(t^{\prime}\right) d t^{\prime}
$$

and

$$
D=N \int_{0}^{+\infty} d \tau \int d^{2} \mathbf{r}_{1} V_{r(t)}(1 \rightarrow 0, t) V_{r(t-\tau)}(1 \rightarrow 0, t-\tau) P_{e q}\left(r_{1}\right) .
$$

The velocity auto-correlation function is calculated in Appendix A under the assumption that between $t$ and $t-\tau$ the point vortices follow the equilibrium streamlines, which is valid for sufficiently strong shears. It is found that the auto-correlation function decreases like $t^{-2}$ at large times so that the diffusion coefficient is well-defined. It must be stressed, however, that the decorrelation is slow so that the fluctuations cannot be described by a white noise process, as indicated previously . Using the results of Appendix A, the final expression for the diffusion coefficient in the presence of a shear can be put in the form 39,36]:

$$
D=\frac{\gamma}{8} \frac{1}{|\Sigma(\mathbf{r})|} \ln N\langle\omega\rangle_{e q}
$$

\footnotetext{
${ }^{1}$ The problem is even more severe in stellar dynamics where the temporal correlation function of the gravitational force decreases like $t^{-1}$, responsible for logarithmic divergences in the diffusion coefficient 88 .
} 
where $|\Sigma(\mathbf{r})|$ is the local shear of the flow: $|\Sigma|=-d / d y\langle V\rangle_{e q}(y)$ for unidirectional flows and $|\Sigma|=r d / d r\left(\langle V\rangle_{e q}(r) / r\right)$ for axisymmetric flows. In Sec. 5.8, we shall calculate the diffusion coefficient in another manner and extend formula (189) to more general equilibrium flows with $|\Sigma(\mathbf{r})|=2 \sqrt{-\operatorname{det}(\Sigma)}$ where $\Sigma$ is the stress tensor. The expression (189) for the diffusion coefficient can be written in the general form (see Sec. 3):

$$
D=\frac{1}{4} \tau\left\langle V^{2}\right\rangle=\frac{\gamma \tau}{16 \pi} \ln N\langle\omega\rangle_{e q},
$$

with a correlation time $\tau=2 \pi /|\Sigma(\mathbf{r})|$. Physically, it corresponds to the time needed by two vortices with relative velocity $\Sigma d$ (where $d$ is the inter-vortex distance) to be stretched by the shear on a distance $\sim d$. Of course, when there is no shear, our approximations break down and the diffusion coefficient is given by Eq. (154) which involves a correlation time $\tau \sim 1 /\langle\omega\rangle(\mathbf{r}) \sqrt{\ln N}$ determined by the dispersion of the vortices [47. These two results correspond to a limit of "strong shear" and "weak shear" respectively. Clearly, a general formula for $D$ should take into account simultaneously the effect of the shear and the dispersion of the vortices.

\subsection{Systematic drift experienced by a point vortex in an inhomogeneous medium: linear response theory}

In Sec. 4.1, we have argued on the basis of general considerations that when the vorticity distribution is inhomogeneous, a point vortex should experience a systematic drift in addition to its diffusive motion. In this section, we justify this drift by a linear response theory, starting directly from the Liouville equation [30. Consider a collection of $N$ point vortices at statistical equilibrium. In the mean-field limit, the N-particle distribution function $\mu_{e q}\left(\left\{\mathbf{r}_{k}\right\}\right)$ can be approximated by a product of $N$ one-particle distribution functions $P_{e q}$, each of which at equilibrium with the same inverse temperature $\beta_{\text {eq }}$ :

$$
\mu_{e q}\left(\left\{\mathbf{r}_{k}\right\}\right)=\prod_{k=1}^{N} P_{e q}\left(\mathbf{r}_{k}\right)=\prod_{k=1}^{N} A e^{-\beta_{e q} \gamma \psi_{e q}\left(\mathbf{r}_{k}\right)} .
$$

This distribution is stationary, in a statistical sense, since it corresponds to a maximum entropy state. The introduction of an additional point vortex, the "test vortex", will modify this equilibrium state. The distribution function of the field vortices becomes time dependant and can be written in the form

$$
\mu\left(\left\{\mathbf{r}_{k}\right\}, t\right)=\mu_{e q}\left(\left\{\mathbf{r}_{k}\right\}\right)+\mu^{\prime}\left(\left\{\mathbf{r}_{k}\right\}, t\right),
$$

where the perturbation $\mu^{\prime}\left(\left\{\mathbf{r}_{k}\right\}, t\right)$ reflects the influence of the test vortex on its neighbors, just like in a polarization process. The $N$-particle distribution function $\mu\left(\left\{\mathbf{r}_{k}\right\}, t\right)$ satisfies the Liouville equation

$$
\frac{\partial \mu}{\partial t}+\sum_{i=1}^{N}\left[\sum_{j \neq i} \mathbf{V}(j \rightarrow i)+\mathbf{V}(0 \rightarrow i)\right] \frac{\partial \mu}{\partial \mathbf{r}_{i}}=0
$$


Substituting Eq. (192) in Eq. (193), we obtain the evolution equation of the perturbation $\mu^{\prime}$ :

$$
\frac{\partial \mu^{\prime}}{\partial t}+\mathcal{L} \mu^{\prime}=\beta_{e q} \gamma \sum_{i=1}^{N} \mathbf{V}_{i} \frac{\partial \psi_{e q}}{\partial \mathbf{r}_{i}}\left(\mathbf{r}_{i}\right) \mu_{e q}\left(\left\{\mathbf{r}_{k}\right\}\right),
$$

where $\mathcal{L} \equiv \sum_{i=1}^{N} \mathbf{V}_{i} \frac{\partial}{\partial \mathbf{r}_{i}}$ is a Liouville operator and $\mathbf{V}_{i}=\sum_{j \neq i} \mathbf{V}(j \rightarrow i)+\mathbf{V}(0 \rightarrow$ $i)$ denotes the total velocity of vortex $i$. This equation can be solved formally with the Greenian

$$
G\left(t, t^{\prime}\right) \equiv \exp \left\{-\int_{t^{\prime}}^{t} \mathcal{L}(\tau) d \tau\right\}
$$

If $t=0$ is the time at which the test vortex is introduced in the system, we have $\mu^{\prime}(t=0)=0$. One then finds that

$$
\mu^{\prime}(t)=\beta_{e q} \gamma \int_{0}^{t} d \tau G(t, t-\tau) \sum_{i=1}^{N} \mathbf{V}_{i} \frac{\partial \psi_{e q}}{\partial \mathbf{r}_{i}}\left(\mathbf{r}_{i}\right) \mu_{e q}\left(\left\{\mathbf{r}_{k}\right\}\right) .
$$

The average velocity of the test vortex is expressed in term of the distribution function $\mu$ of the field vortices by

$$
\langle\mathbf{V}\rangle=\int \prod_{k=1}^{N} d^{2} \mathbf{r}_{k} \mathbf{V} \mu\left(\left\{\mathbf{r}_{k}\right\}, t\right),
$$

where $\mathbf{V}=\sum_{i=1}^{N} \mathbf{V}(i \rightarrow 0)$. Inserting Eqs. (192) and (196) into Eq. 197), one obtains

$$
\begin{aligned}
& \langle\mathbf{V}\rangle=\int \prod_{k=1}^{N} d^{2} \mathbf{r}_{k} \mathbf{V} \mu_{e q}\left(\left\{\mathbf{r}_{k}\right\}\right)+\beta_{e q} \gamma \int \prod_{k=1}^{N} d^{2} \mathbf{r}_{k} \mathbf{V} \\
& \times \int_{0}^{t} d \tau G(t, t-\tau) \sum_{i=1}^{N} V_{i}^{\nu} \frac{\partial \psi_{e q}}{\partial r_{i}^{\nu}}\left(\mathbf{r}_{i}\right) \mu_{e q}\left(\left\{\mathbf{r}_{k}\right\}\right),
\end{aligned}
$$

with summation over repeated greek indices. The two terms arising in this expression have a clear physical meaning. The first term is the mean field velocity $\langle\mathbf{V}\rangle_{e q}=-\mathbf{z} \times \nabla \psi_{e q}(\mathbf{r})$ created by the unperturbed distribution function $\mu_{e q}\left(\left\{\mathbf{r}_{k}\right\}\right)$. The second term, arising from the perturbation $\mu^{\prime}$, corresponds to the response of the system to the polarization induced by the test vortex. Because of this back reaction, the test vortex will experience a systematic drift $\langle\mathbf{V}\rangle_{\text {drift }}=\langle\mathbf{V}\rangle-\langle\mathbf{V}\rangle_{e q}$. Explicating the action of the Greenian (195), we obtain

$$
\begin{aligned}
& \langle\mathbf{V}\rangle_{d r i f t}=\beta_{e q} \gamma \int \prod_{k=1}^{N} d^{2} \mathbf{r}_{k} \sum_{i=1}^{N} \mathbf{V}(i \rightarrow 0, t) \int_{0}^{t} d \tau \sum_{i=1}^{N}\left[\sum_{j \neq i} V^{\nu}(j \rightarrow i, t-\tau)\right. \\
& \left.+V^{\nu}(0 \rightarrow i, t-\tau)\right] \frac{\partial \psi_{e q}}{\partial r_{i}^{\nu}}\left(\mathbf{r}_{i}(t-\tau)\right) \mu_{e q}\left(\left\{\mathbf{r}_{k}(t-\tau)\right\}\right)
\end{aligned}
$$


where $\mathbf{r}_{i}(t-\tau)$ is the position at time $t-\tau$ of the point vortex $i$ located at $\mathbf{r}_{i}(t)=\mathbf{r}_{i}$ at time $t$. This is obtained by solving the Kirchhoff-Hamilton equations of motion $d \mathbf{r}_{i} / d t=\mathbf{V}_{i}$ between $t$ and $t-\tau$.

The exact expression of the drift (199) is completely inextricable in the general case. In order to enlighten its physical content, we have to make some approximations. We shall consider in the evaluation of the time integral that the point vortices are advected by the equilibrium mean-field velocity $\langle\mathbf{V}\rangle_{e q}$. This is reasonable in a first approximation because, when $N \rightarrow \infty$, the typical velocity fluctuations $\mathcal{V}$, of order $\gamma / d \sim \gamma N^{1 / 2} / R$ are much smaller than the mean field velocity $\langle\mathbf{V}\rangle_{e q}$, of order $N \gamma / R$. Of course, this approximation breaks up at scales smaller than $\delta \sim R / N$ when the velocity fluctuations become comparable to the average velocity. In that case, we cannot ignore the details of the discrete vortex interactions anymore and a specific treatment is necessary. For simplicity, we shall introduce a small-scale cut-off and replace the exact Greenian $G$ by a smoother Greenian $\langle G\rangle_{e q}$ constructed with the averaged Liouville operator $\langle\mathcal{L}\rangle_{e q} \equiv \sum_{i=1}^{N}\left\langle\mathbf{V}^{i}\right\rangle_{e q} \frac{\partial}{\partial \mathbf{r}_{i}}$. In this approximation the correlations involving two different vortex pairs vanish and we obtain

$$
\begin{aligned}
& \left\langle V^{\mu}\right\rangle_{d r i f t}=\beta_{e q} \gamma \int \prod_{k=1}^{N} d^{2} \mathbf{r}_{k} \int_{0}^{t} d \tau \sum_{i=1}^{N} V^{\mu}(i \rightarrow 0, t) \\
& \times V^{\nu}(0 \rightarrow i, t-\tau) \frac{\partial \psi_{e q}}{\partial r_{i}^{\nu}}\left(\mathbf{r}_{i}(t-\tau)\right) \prod_{i=k}^{N} P_{e q}\left(\mathbf{r}_{k}\right)
\end{aligned}
$$

where we have used $P^{e q}\left(\mathbf{r}_{k}(t-\tau)\right)=P^{e q}\left(\mathbf{r}_{k}(t)\right)$ since $P_{e q}=f\left(\psi_{e q}\right)$ is constant along a streamline. Since the vortices are identical, we have equivalently

$$
\begin{aligned}
& \left\langle V^{\mu}\right\rangle_{d r i f t}=N \beta_{e q} \gamma \int d^{2} \mathbf{r}_{1} \int_{0}^{t} d \tau V^{\mu}(1 \rightarrow 0, t) \\
& \times V^{\nu}(0 \rightarrow 1, t-\tau) \frac{\partial \psi_{e q}}{\partial r_{1}^{\nu}}\left(\mathbf{r}_{1}(t-\tau)\right) P_{e q}\left(\mathbf{r}_{1}\right)
\end{aligned}
$$

In the case of a unidirectional equilibrium flow in the $x$ direction, the drift velocity 201 can be written

$$
\left\langle V_{y}\right\rangle_{d r i f t}=N \beta_{e q} \gamma \int d^{2} \mathbf{r}_{1} \int_{0}^{+\infty} d \tau V_{y}(1 \rightarrow 0, t) V_{y}(0 \rightarrow 1, t-\tau) \frac{\partial \psi_{e q}}{\partial y_{1}}\left(y_{1}\right) P_{e q}\left(y_{1}\right),
$$

where we have used $y_{1}(t-\tau)=y_{1}(t)=y_{1}$ and the time integration has been extended to $+\infty$. Since the space integration diverges as $\mathbf{r}_{1} \rightarrow \mathbf{r}_{0}$, we can make a local approximation $\partial_{y} \psi\left(y_{1}\right) \simeq \partial_{y} \psi(y)$, neglect the contribution of images in the velocity Kernel and use $V_{y}(0 \rightarrow 1)=-V_{y}(1 \rightarrow 0)$. The local approximation reflects the strong influence of the nearest neighbor and is only marginally valid as discussed in Sec. 3. The expression of the drift can then be written

$$
\left\langle V_{y}\right\rangle_{d r i f t}=-\beta_{e q} \gamma D \frac{\partial \psi_{e q}}{\partial y}(y)
$$


where $D$ is the diffusion coefficient given by formula (186). The same type of relation is obtained for an axisymmetric equilibrium flow. More generaly, one can write the drift as 30,36]:

$$
\langle\mathbf{V}\rangle_{\text {drift }}=-\beta_{e q} \gamma D \nabla \psi_{e q},
$$

where $D$ is given by Eq. (189). We have thus derived, from a kinetic theory, an Einstein relation $\xi=D \gamma \beta_{e q}$ for the point vortex gas. This relation is of great conceptual importance and could be checked by direct numerical simulations of point vortex dynamics in the thermal bath approach.

The direction of the drift has also important physical implications. First, we note that the drift is always normal to the mean field velocity $\langle\mathbf{V}\rangle_{e q}=-\mathbf{z} \times \nabla \psi_{e q}$. Since $P_{e q}=f\left(\psi_{e q}\right)$ for an equilibrium flow, this implies that the drift is directed along the vorticity gradient. This is clear at first sight if we write the expression of the drift in the form $\langle\mathbf{V}\rangle_{\text {drift }}=D \nabla \ln \langle\omega\rangle_{e q}$, using Eqs. (204) and (44). Since $D \geq 0$, this formula indicates that the test vortex always ascends the vorticity gradient. In fact, we have assumed in the previous discussion that all the vortices have the same circulation. It is straightforward to generalize our calculations for a test vortex with negative circulation evolving in a bath of vortices with positive circulations. Similar results are obtained except that now the test vortex descends the vorticity gradient.

If we now take into account simultaneously the drift and the diffusion of the test vortex, we can argue that the evolution of the density probability $P(\mathbf{r}, t)$ is governed by the Fokker-Planck equation

$$
\frac{\partial P}{\partial t}+\langle\mathbf{V}\rangle_{e q} \nabla P=\nabla\left(D\left(\nabla P+\beta_{e q} \gamma P \nabla \psi_{e q}\right)\right),
$$

where we recall that $\psi_{e q}$ is the stationary streamfunction generated by the equilibrium vorticity $\langle\omega\rangle_{e q}$ via the Poisson equation (7). In the case of a unidirectional flow, this Fokker-Planck equation can be transformed into a Schrödinger equation (with imaginary time) which can be solved analytically [116].

\subsection{A relaxation equation for point vortices}

If we are sufficiently close to equilibrium, we can try to apply our previous results to all vortices in the system, eliminating the somewhat arbitrary distinction between test and field vortices. We propose to describe the relaxation of a cloud of point vortices towards statistical equilibrium by the following set of equations

$$
\begin{gathered}
\frac{\partial P}{\partial t}+\langle\mathbf{V}\rangle \nabla P=\nabla(D(\nabla P+\beta \gamma P \nabla \psi)), \\
\Delta \psi=-N \gamma P,
\end{gathered}
$$

consisting of the Fokker-Planck equation (205) coupled to the Poisson equation (77). This model is expected to be valid only close to equilibrium so that the inverse temperature $\beta$ has a clear physical interpretation. In this model, a point 
vortex is assumed to undergo a diffusion process due to the fluctuations of the velocity and a systematic drift $\langle\mathbf{V}\rangle_{\text {drift }}=-\beta \gamma D \nabla \psi$ due to the inhomogeneity of the vortex cloud. At negative temperatures, the drift is directed inward and the vortices tend to cluster while at positive temperatures, the drift is directed outward and the vortices tend to repell each other and accumulate on the boundary. For $\beta=0$, there is no drift and Eq. (206) reduces to the pure diffusion equation (180); in that case, the vorticity distribution is uniform in average. These results are consistent with the thermodynamical approach of Onsager [101] but the drift provides a physical mechanism to understand the clustering of point vortices at negative temperatures. It can also be noted that Eq. (206) is formally similar to the Smoluchowski equation describing the relaxation of colloidal suspensions in an external gravitational field 108]. In the present context, however, the field $\psi$ is not fixed but is generated by the distribution of particles itself via the Poisson equation. The resulting Smoluchowski-Poisson system has been studied in 46. 116 for various space dimensions.

The system of equations (206) and (207) is also similar to the model introduced by Chandrasekhar 23] in his stochastic description of stellar dynamics:

$$
\begin{gathered}
\frac{\partial f}{\partial t}+\mathbf{v} \frac{\partial f}{\partial \mathbf{r}}+\langle\mathbf{F}\rangle \frac{\partial f}{\partial \mathbf{v}}=\frac{\partial}{\partial \mathbf{v}}\left\{D\left(\frac{\partial f}{\partial \mathbf{v}}+\beta m f \mathbf{v}\right)\right\} \\
\Delta \Phi=4 \pi G \int f d^{3} \mathbf{v}
\end{gathered}
$$

In that model, a star undergoes a diffusion process (in velocity space) due to the fluctuations of the gravitational force and a dynamical friction $\langle\mathbf{F}\rangle_{\text {friction }}=$ $-D \beta m \mathbf{v}$ resulting from close encounters. Fundamentally, this friction is due to the inhomogeneity of the velocity distribution. The coefficient of dynamical friction is given by an Einstein formula $\xi=D \beta m$ in which the velocity dispersion $1 / \beta$ of the stars enters explicitly.

The morphological similarity of the two models (206)-(207) and (208)-(209) is striking although the physical content of these equations is, of course, very different. In this analogy, we see that the systematic drift of the vortices is the counterpart of the dynamical friction of stars. In fact, Chandrasekhar's dynamical friction can be derived from a linear response theory 74 exactly like we have derived the systematic drift of a vortex. In addition, both terms can be understood physically as a result of a polarization process (see discussion in [36]). This is another mark of the formal analogy between point vortices and stars.

\section{$5 \quad$ Kinetic theory of point vortices}

The previous relaxation equations are only valid for a test vortex evolving in a bath of field vortices, or for a cloud of point vortices close to statistical equilibrium. We would like now to relax this "thermal bath approximation" and describe more general situations which do not explicitly rely on the existence of a well-defined temperature or equilibrium state. Hence, we would like to develop a more complete kinetic theory of point vortices [36]. 


\subsection{The Liouville equation}

Let us consider a collection of $N+1$ point vortices with identical circulation $\gamma$. Let $\mu\left(\mathbf{r}, \mathbf{r}_{1}, \ldots, \mathbf{r}_{N}, t\right)$ denote the $N+1$ particle distribution of the system, i.e. $\mu\left(\mathbf{r}, \mathbf{r}_{1}, \ldots, \mathbf{r}_{N}, t\right) d^{2} \mathbf{r} d^{2} \mathbf{r}_{1} \ldots d^{2} \mathbf{r}_{N}$ represents the probability that point vortex 0 be in the cell $(\mathbf{r}, \mathbf{r}+d \mathbf{r})$, point vortex 1 in the cell $\left(\mathbf{r}_{1}, \mathbf{r}_{1}+d \mathbf{r}_{1}\right) \ldots$ and point vortex $N$ in the cell $\left(\mathbf{r}_{N}, \mathbf{r}_{N}+d \mathbf{r}_{N}\right)$ at time $t$. The $(N+1)$-particle distribution function $\mu(t)$ satisfies the Liouville equation

$$
\frac{\partial \mu}{\partial t}+\sum_{i=0}^{N} \mathbf{V}_{i} \frac{\partial \mu}{\partial \mathbf{r}_{i}}=0
$$

where $\mathbf{V}_{i}$ is the velocity of vortex $i$ produced by the other vortices according to Eq. (14). We also introduce the one- and $N$-particle distribution functions defined by

$$
\begin{gathered}
P(\mathbf{r}, t)=\int \mu\left(\left\{\mathbf{r}_{k}\right\}, t\right) \prod_{k=1}^{N} d^{2} \mathbf{r}_{k}, \\
\mu_{s y s}\left(\mathbf{r}_{1}, \ldots, \mathbf{r}_{N}, t\right)=\int \mu\left(\left\{\mathbf{r}_{k}\right\}, t\right) d^{2} \mathbf{r} .
\end{gathered}
$$

We write the distribution function $\mu$ in the suggestive form

$$
\mu\left(\mathbf{r}, \mathbf{r}_{1}, \ldots, \mathbf{r}_{N}, t\right)=P(\mathbf{r}, t) \mu_{s y s}\left(\mathbf{r}_{1}, \ldots, \mathbf{r}_{N}, t\right)+\mu_{I}\left(\mathbf{r}, \mathbf{r}_{1}, \ldots, \mathbf{r}_{N}, t\right),
$$

where the quantity $\mu_{I}$ reflects the effect of correlations between vortices.

The Liouville equation (210) provides the correct starting point for the analysis of the dynamics of our vortex system. However, when $N$ is large, this equation contains much more information than one can interpret. Consequently, what one would like to do is to describe the system in some average sense by a one-particle distribution function.

\subsection{The projection operator formalism}

Our first objective is to derive some exact kinetic equations satisfied by $P(\mathbf{r}, t)$ and $\mu_{\text {sys }}\left(\mathbf{r}_{1}, \ldots, \mathbf{r}_{N}, t\right)$. This can be achieved by using the projection operator formalism developed by Willis \& Picard 127]. This formalism was also used by Kandrup 773 in the context of stellar dynamics to derive a generalized Landau equation describing the time evolution of the distribution function of stars in an inhomogeneous medium. We shall just recall the main steps of the theory. More details can be found in the original paper of Willis \& Picard [127] and in Kandrup [73|. To have similar notations, we set $x \equiv\{\mathbf{r}\}$ and $y \equiv\left\{\mathbf{r}_{1}, \ldots, \mathbf{r}_{N}\right\}$. Then, Eq. (213) can be put in the form

$$
\mu(x, y, t)=\mu_{R}(x, y, t)+\mu_{I}(x, y, t),
$$

with

$$
\mu_{R}(x, y, t)=f(x, t) g(y, t),
$$


where we have written $f(x, t) \equiv P(\mathbf{r}, t)$ and $g(y, t) \equiv \mu_{\text {sys }}\left(\mathbf{r}_{1}, \ldots, \mathbf{r}_{N}, t\right)$. The Liouville equation is also cast in the form

$$
\frac{\partial \mu}{\partial t}=-i L \mu=-i\left(L_{0}+L_{s y s}+L^{\prime}\right) \mu
$$

where $L_{0}$ and $L_{\text {sys }}$ act respectively only on the variables $x$ and $y$, whereas the interaction Liouvillian $L^{\prime}$ acts upon both $x$ and $y$ (the complex number $i$ is here purely formal and has been introduced only to have the same notations as in 127.73 ).

Following Willis \& Picard [127, we introduce the time-dependant projection operator

$$
P(x, y, t)=g(y, t) \int d y+f(x, t) \int d x-f(x, t) g(y, t) \int d x \int d y .
$$

We can easily check that

$$
\begin{gathered}
P(x, y, t) \mu(x, y, t)=\mu_{R}(x, y, t), \\
{[1-P(x, y, t)] \mu(x, y, t)=\mu_{I}(x, y, t) .}
\end{gathered}
$$

We also verify that $P$ is a projection in the sense that $P^{2}(t)=P(t)$. Applying $P$ and $1-P$ on the Liouville equation (216), we obtain the coupled equations

$$
\partial_{t} \mu_{R}(x, y, t)=-i P L \mu_{R}-i P L \mu_{I},
$$

and

$$
\partial_{t} \mu_{I}(x, y, t)=-i(1-P) L \mu_{R}-i(1-P) L \mu_{I} .
$$

These equations describe the separation between a "macrodynamics" and a "subdynamics".

Introducing the Greenian

$$
\mathcal{G}\left(t, t^{\prime}\right) \equiv \exp \left\{-i \int_{t^{\prime}}^{t} d t^{\prime \prime}\left[1-P\left(t^{\prime \prime}\right)\right] L\right\},
$$

we can immediately write down a formal solution of Eq. (221), namely

$$
\mu_{I}(x, y, t)=-\int_{0}^{t} d t^{\prime} \mathcal{G}\left(t, t^{\prime}\right) i\left[1-P\left(t^{\prime}\right)\right] L \mu_{R}\left(x, y, t^{\prime}\right),
$$

where we have assumed that, initially, the particles are uncorrelated so that $\mu_{I}(x, y, 0)=0$. Substituting for $\mu_{I}(x, y, t)$ from Eq. (223) in Eq. (220), we obtain

$$
\partial_{t} \mu_{R}(x, y, t)=-i P L \mu_{R}-\int_{0}^{t} d t^{\prime} P(t) L \mathcal{G}\left(t, t^{\prime}\right)\left[1-P\left(t^{\prime}\right)\right] L \mu_{R}\left(x, y, t^{\prime}\right)
$$


The integration over $y$ will yield an equation describing the evolution of $f$. Using some mathematical properties of the projection operator (217), the final result can be put in the nice symmetrical form given by Willis \& Picard [127],

$$
\partial_{t} f(x, t)+i L_{0} f+i\left\langle L^{\prime}\right\rangle_{s y s} f=-\int_{0}^{t} d t^{\prime} \int d y \Delta_{t} L^{\prime} \mathcal{G}\left(t, t^{\prime}\right) \Delta_{t^{\prime}} L^{\prime} g\left(y, t^{\prime}\right) f\left(x, t^{\prime}\right)
$$

where the notations stand for

$$
\begin{aligned}
\left\langle L^{\prime}\right\rangle_{s y s} & =\int d y^{\prime} L^{\prime}\left(x, y^{\prime}\right) g\left(y^{\prime}, t\right), \\
\left\langle L^{\prime}\right\rangle_{0} & =\int d x^{\prime} L^{\prime}\left(x^{\prime}, y\right) f\left(x^{\prime}, t\right), \\
\Delta_{t} L^{\prime} & =L^{\prime}-\left\langle L^{\prime}\right\rangle_{s y s}-\left\langle L^{\prime}\right\rangle_{0} .
\end{aligned}
$$

Similarly, after integrating over $x$ we find the equation satisfied by $g$,

$$
\partial_{t} g(y, t)+i L_{s y s} g-i\left\langle L^{\prime}\right\rangle_{1} g=-\int_{0}^{t} d t^{\prime} \int d x \Delta_{t} L^{\prime} \mathcal{G}\left(t, t^{\prime}\right) \Delta_{t^{\prime}} L^{\prime} g\left(y, t^{\prime}\right) f\left(x, t^{\prime}\right)
$$

\subsection{Application to the point vortex system}

The previous theory is completely general and we now consider its application to a system of point vortices [36. Let us first rewrite the Liouville equation (210) in a form that separates the contribution of the test vortex from the contribution of the field vortices:

$$
\begin{aligned}
& \frac{\partial \mu}{\partial t}+\sum_{i=1}^{N} \mathbf{V}(i \rightarrow 0) \frac{\partial \mu}{\partial \mathbf{r}}+\sum_{i=1}^{N} \mathbf{V}(0 \rightarrow i) \frac{\partial \mu}{\partial \mathbf{r}_{i}} \\
& +\sum_{i=1}^{N} \sum_{j=1, j \neq i}^{N} \mathbf{V}(j \rightarrow i) \frac{\partial \mu}{\partial \mathbf{r}_{i}}=0 .
\end{aligned}
$$

Applying the general theory of Willis \& Picard [127, we obtain the following kinetic equation for the one-particle distribution function of a vortex system [36]:

$$
\begin{aligned}
& \frac{\partial P}{\partial t}+\langle\mathbf{V}\rangle \frac{\partial P}{\partial \mathbf{r}}=\frac{\partial}{\partial r^{\mu}} \int_{0}^{t} d \tau \int \prod_{k=1}^{N} d^{2} \mathbf{r}_{k} \sum_{i=1}^{N} \sum_{j=1}^{N} \mathcal{V}^{\mu}(i \rightarrow 0) \\
& \times \mathcal{G}(t, t-\tau)\left(\mathcal{V}^{\nu}(j \rightarrow 0) \frac{\partial}{\partial r^{\nu}}+\mathcal{V}^{\nu}(0 \rightarrow j) \frac{\partial}{\partial r_{j}^{\nu}}\right) P(\mathbf{r}, t-\tau) \mu_{s y s}\left(\left\{\mathbf{r}_{k}\right\}, t-\tau\right),
\end{aligned}
$$

where the Greek indices refer to the components of $\mathcal{V}$ in a fixed system of coordinates and $\mathcal{V}(i \rightarrow 0)=\mathbf{V}(i \rightarrow 0)-\langle\mathbf{V}(i \rightarrow 0)\rangle$ denotes the velocity fluctuation. We can note that equation (231) already shares some analogies with the Fokker-Planck equation of Sec. 1. Indeed, the first term on the right hand side corresponds to a diffusion and the second term to a drift. For a passive particle $\mathcal{V}^{\nu}(0 \rightarrow j)=0$ and the drift cancels out, as expected. 


\subsection{The factorization hypothesis}

If the vortices are initially decorrelated then, for sufficiently short times, they will remain decorrelated. This means that the $(N+1)$-particle distribution function can be factorized in a product of $(N+1)$ one-particle distribution functions

$$
\mu\left(\mathbf{r}, \mathbf{r}_{1}, \ldots, \mathbf{r}_{N}, t\right)=\prod_{k=0}^{N} P\left(\mathbf{r}_{k}, t\right) .
$$

If we integrate the Liouville equation (210) on the positions of the $N$ vortices $1, \ldots, N$ and use the factorization (232), we directly obtain 36:

$$
\frac{\partial P}{\partial t}+\langle\mathbf{V}\rangle \nabla P=0
$$

Therefore, for sufficiently short times, the average vorticity $\langle\omega\rangle$ satisfies the $2 \mathrm{D}$ Euler equation (called the Vlasov equation in other circumstances). However, at later times, the distribution function $\mu$ differs from the pure product (232) and the Euler equation does not provide a good approximation anymore. In Sec. 5.3 we have determined an exact equation (231) satisfied by the one-particle distribution function which is valid at any time. This equation is not closed, however, since it involves the $N$-vortex distribution function $\mu_{\text {sys }}$. We shall close the system by assuming that $\mu_{\text {sys }}$ can be approximated by a product of $\mathrm{N}$ oneparticle distribution functions in the form

$$
\mu_{\text {sys }}\left(\mathbf{r}_{1}, \ldots, \mathbf{r}_{N}, t\right) \simeq \prod_{k=1}^{N} P\left(\mathbf{r}_{k}, t\right) .
$$

Inserting Eq. (234) in Eq. (231), we obtain

$$
\begin{aligned}
& \frac{\partial P}{\partial t}+\langle\mathbf{V}\rangle \frac{\partial P}{\partial \mathbf{r}}=\frac{\partial}{\partial r^{\mu}} \int \prod_{k=1}^{N} d^{2} \mathbf{r}_{k} \int_{0}^{t} d \tau \sum_{i=1}^{N} \sum_{j=1}^{N} \mathcal{V}^{\mu}(i \rightarrow 0) \\
& \times \mathcal{G}(t, t-\tau)\left(\mathcal{V}^{\nu}(j \rightarrow 0) \frac{\partial}{\partial r^{\nu}}+\mathcal{V}^{\nu}(0 \rightarrow j) \frac{\partial}{\partial r_{j}^{\nu}}\right) P(\mathbf{r}, t-\tau) \prod_{k=1}^{N} P\left(\mathbf{r}_{k}, t-\tau\right) .
\end{aligned}
$$

If we assume that between $t$ and $t-\tau$ the trajectories of the particles are determined from the smooth velocity field created by the vorticity distribution $\langle\omega\rangle=N \gamma P(\mathbf{r}, t)$, the foregoing equation simplifies in

$$
\begin{aligned}
& \frac{\partial P}{\partial t}+\langle\mathbf{V}\rangle \frac{\partial P}{\partial \mathbf{r}}=N \frac{\partial}{\partial r^{\mu}} \int_{0}^{t} d \tau \int d^{2} \mathbf{r}_{1} V^{\mu}(1 \rightarrow 0)_{t} \\
& \times\left\{V^{\nu}(1 \rightarrow 0) P_{1} \frac{\partial P}{\partial r^{\nu}}+V^{\nu}(0 \rightarrow 1) P \frac{\partial P_{1}}{\partial r_{1}^{\nu}}\right\}_{t-\tau},
\end{aligned}
$$


where $P=P(\mathbf{r}, t)$ and $P_{1}=P\left(\mathbf{r}_{1}, t\right)$. Eq. (236) is a non Markovian integrodifferential equation since the probability density $P(\mathbf{r}, t)$ in $\mathbf{r}$ at time $t$ depends on the value of the whole distribution of probability $P\left(\mathbf{r}_{1}, t-\tau\right)$ at earlier times through an integration on $\mathbf{r}_{1}$ and $\tau$. Equation (236) is therefore non local in space and time. It can be shown [36 that this kinetic equation rigorously conserves angular momentum in a circular domain and linear impulse in a channel (or in an infinite domain). However, under this form, it has not been possible to prove the conservation of energy and the H-theorem for the Boltzmann entropy (41).

\subsection{The case of short decorrelation times}

If we assume that the decorrelation time $\tau$ is short (which does not need to be the case) and implement a strong Markov approximation, we obtain

$$
\begin{aligned}
& \frac{\partial P}{\partial t}+\langle\mathbf{V}\rangle \frac{\partial P}{\partial \mathbf{r}}=\frac{N \tau}{2} \frac{\partial}{\partial r^{\mu}} \int d^{2} \mathbf{r}_{1} V^{\mu}(1 \rightarrow 0) \\
& \times\left\{V^{\nu}(1 \rightarrow 0) P_{1} \frac{\partial P}{\partial r^{\nu}}+V^{\nu}(0 \rightarrow 1) P \frac{\partial P_{1}}{\partial r_{1}^{\nu}}\right\} .
\end{aligned}
$$

In the case of an infinite domain $\mathbf{V}(0 \rightarrow 1)=-\mathbf{V}(0 \rightarrow 1)$ and we have the further simplification

$$
\frac{\partial P}{\partial t}+\langle\mathbf{V}\rangle \frac{\partial P}{\partial \mathbf{r}}=\frac{N \gamma^{2}}{8 \pi^{2}} \tau \frac{\partial}{\partial r^{\mu}} \int d^{2} \mathbf{r}_{1} K^{\prime \mu \nu}(\boldsymbol{\xi})\left(P_{1} \frac{\partial P}{\partial r^{\nu}}-P \frac{\partial P_{1}}{\partial r_{1}^{\nu}}\right),
$$

where

$$
K^{\prime \mu \nu}(\boldsymbol{\xi})=\frac{\xi_{\perp}^{\mu} \xi_{\perp}^{\nu}}{\xi^{4}}=\frac{\xi^{2} \delta^{\mu \nu}-\xi^{\mu} \xi^{\nu}}{\xi^{4}}
$$

and $\boldsymbol{\xi}=\mathbf{r}_{1}-\mathbf{r}$. To arrive at Eq. (239) we have explicitly used the form of the Kernel (14), and to get the second equality we have used the fact that we are in two dimensions. Note that the symmetrical form of Eq. (238) is reminiscent of the Landau equation introduced in plasma physics and in stellar dynamics (see, e.g., [73):

$$
\frac{\partial f}{\partial t}=\frac{\partial}{\partial v^{\mu}} \int d^{3} \mathbf{v}_{1} K^{\mu \nu}\left(f_{1} \frac{\partial f}{\partial v^{\nu}}-f \frac{\partial f_{1}}{\partial v_{1}^{\nu}}\right)
$$

with

$$
K^{\mu \nu}=2 \pi N G^{2} m^{2} \ln \left(\frac{L_{\max }}{L_{\min }}\right) \frac{u^{2} \delta^{\mu \nu}-u^{\mu} u^{\nu}}{u^{3}},
$$

and $\mathbf{u}=\mathbf{v}-\mathbf{v}_{\mathbf{1}}, f=f(\mathbf{v}, t), f_{1}=f\left(\mathbf{v}_{1}, t\right)$. In this analogy, the position $\mathbf{r}$ of the vortices plays the role of the velocity $\mathbf{v}$ of the electric charges or stars and the spatial distribution $P(\mathbf{r}, t)$ the role of the velocity distribution $f(\mathbf{v}, t)$. Therefore, we can directly infer the conservation of linear impulse $\mathbf{P}_{\perp}=\int\langle\omega\rangle \mathbf{r} d^{2} \mathbf{r}$ and 
angular momentum $L=\int\langle\omega\rangle r^{2} d^{2} \mathbf{r}$ which play respectively the role of impulse $\mathbf{P}=\int f \mathbf{v} d^{3} \mathbf{v}$ and kinetic energy $K=\int f \frac{v^{2}}{2} d^{3} \mathbf{v}$ in plasma physics. We can also prove a $H$-theorem for the Boltzmann entropy (41) exactly like in the case of the Landau equation. Finally, the solutions of Eq. (238) converge towards the Gaussian vortex (the equivalent of the Maxwellian distribution in plasma physics with $\mathbf{r}$ in place of $\mathbf{v})$ :

$$
P(\mathbf{r})=A e^{-\frac{1}{2} \alpha \gamma\left(\mathbf{r}-\mathbf{r}_{0}\right)^{2}}
$$

which is the maximum entropy state at fixed circulation, angular momentum and impulse. It is in general different from the Boltzmann distribution (44) with the relative streamfunction $\psi^{\prime}=\psi+\frac{\Omega}{2} r^{2}-\mathbf{U}_{\perp} \mathbf{r}$, except in the particular limit $\beta \rightarrow 0, \Omega \rightarrow+\infty$ with fixed $\alpha=\beta \Omega / 2$ corresponding to the statistical equilibrium (88). This clearly indicates that Eq. 238) does not conserve energy.

\subsection{A generalized kinetic equation}

Now, if we account properly for memory effects in Eq. (236), we can obtain a generalized kinetic equation which guaranties the conservation of energy (in addition to the other constraints) and is therefore more satisfactory. If the distribution of vortices is axisymmetric, it is possible to calculate the memory function appearing in Eq. (236) explicitly if we assume that the correlation time is smaller than the typical time on which the average vorticity changes appreciably 36]. In this approximation, the point vortices follow, between $t$ and $t-\tau$, circular trajectories with angular velocity $\Omega(r, t)=\left\langle V_{\theta}\right\rangle(r, t) / r$ and Eq. 236) simplifies in (see Appendix B):

$$
\frac{\partial P}{\partial t}=-\frac{N \gamma^{2}}{4 r} \frac{\partial}{\partial r} \int_{0}^{+\infty} r_{1} d r_{1} \delta\left(\Omega-\Omega_{1}\right) \ln \left[1-\left(\frac{r_{<}}{r_{>}}\right)^{2}\right]\left\{\frac{1}{r} P_{1} \frac{\partial P}{\partial r}-\frac{1}{r_{1}} P \frac{\partial P_{1}}{\partial r_{1}}\right\}
$$

where $\Omega=\Omega(r, t), \Omega_{1}=\Omega\left(r_{1}, t\right)$ and $r_{>}$(resp. $\left.r_{<}\right)$is the biggest (resp. smallest) of $r$ and $r_{1}$. The angular velocity is related to the vorticity by

$$
\langle\omega\rangle=\frac{1}{r} \frac{\partial}{\partial r}\left(\Omega r^{2}\right) .
$$

We can propose an approximation of the general kinetic equation (236) which encompasses the axisymmetric form previously derived. Memory effects are not neglected, unlike in Eq. (238), but they are simplified in a way which preserves all the conservation laws of the system (as discussed below). We propose the generalized kinetic equation [36]:

$$
\frac{\partial P}{\partial t}+\langle\mathbf{V}\rangle \nabla P=\frac{N \gamma^{2}}{8} \frac{\partial}{\partial r^{\mu}} \int d^{2} \mathbf{r}_{1} K^{\mu \nu} \delta(\boldsymbol{\xi} . \mathbf{v})\left(P_{1} \frac{\partial P}{\partial r^{\nu}}-P \frac{\partial P_{1}}{\partial r_{1}^{\nu}}\right)
$$

with

$$
K^{\mu \nu}(\boldsymbol{\xi})=\frac{\xi_{\perp}^{\mu} \xi_{\perp}^{\nu}}{\xi^{2}}=\frac{\xi^{2} \delta^{\mu \nu}-\xi^{\mu} \xi^{\nu}}{\xi^{2}}
$$


and $\boldsymbol{\xi}=\mathbf{r}_{1}-\mathbf{r}, \mathbf{v}=\langle\mathbf{V}\rangle\left(\mathbf{r}_{1}, t\right)-\langle\mathbf{V}\rangle(\mathbf{r}, t)$. In the thermodynamic limit $N \rightarrow+\infty$ and $\gamma \sim 1 / N$ (see Sec. 2.7), the kinetic equation (245) reduces to the Vlasov equation (233). However, in practice, $N$ is always finite and the correlations between point vortices must be taken into account. The "collision term" in Eq. (245) gives the first order correction $O(1 / N)$ to the Vlasov limit.

From Eqs. (243) and (245), it is clear that the relaxation of the point vortices is due to a phenomenon of resonance. Only the points $\mathbf{r}_{1}$ satisfying the condition $\boldsymbol{\xi} \cdot \mathbf{v}=0$ with $\mathbf{r}_{1} \neq \mathbf{r}$ contribute to the diffusion current in $\mathbf{r}$. In the axisymmetric case, this condition of resonance reduces to $\Omega\left(r_{1}\right)=\Omega(r)$, which supposes that the angular velocity profile is non monotonic. The occurence of a $\delta$-function in Eqs. (243) and (245) is the main difference with the Landau equation 240. In the present context, it ensures the conservation of the "potential" energy of the vortices $E=\frac{1}{2} \int \omega \psi d^{2} \mathbf{r}$ which has no counterpart in Landau's theory applying to spatially uniform plasmas. It can also be noted that, contrary to the Landau equation, the kinetic equations (238) and (245) do not suffer the well-known logarithmic divergence appearing in the context of Coulombian plasmas and stellar systems (see, e.g., 730). This is due essentially to the lower dimension of space $(D=2$ instead of $D=3$ ) and to the different nature of the interactions.

\subsection{Conservation laws and H-theorem}

We now derive the conservation laws and the H-theorem satisfied by Eq. (245). The conservation of the circulation is straightforward since the right hand side of Eq. (245) can be written as the divergence of a current. To prove the conservation of angular momentum, we take the time derivative of $L=N \gamma \int \operatorname{Pr}^{2} d^{2} \mathbf{r}$, substitute for Eq. (245), permut the dummy variables $\mathbf{r}$ and $\mathbf{r}_{1}$ and add the resulting expressions. This yields

$$
\dot{L}=\frac{N^{2} \gamma^{3}}{8} \int d^{2} \mathbf{r} d^{2} \mathbf{r}_{1} K^{\mu \nu} \xi^{\mu} \delta(\boldsymbol{\xi} . \mathbf{v})\left(P_{1} \frac{\partial P}{\partial r^{\nu}}-P \frac{\partial P_{1}}{\partial r_{1}^{\nu}}\right)
$$

From Eq. (246), we immediately verify that

$$
K^{\mu \nu} \xi^{\mu}=0
$$

which proves the conservation of angular momentum. We can prove the conservation of linear impulse in a similar manner [36]. For the conservation of energy, we start from Eq. (39) and follow the same procedure. This yields

$$
\dot{E}=\frac{N^{2} \gamma^{3}}{16} \int d^{2} \mathbf{r} d^{2} \mathbf{r}_{1} K^{\mu \nu} v_{\perp}^{\mu} \delta(\boldsymbol{\xi} . \mathbf{v})\left(P_{1} \frac{\partial P}{\partial r^{\nu}}-P \frac{\partial P_{1}}{\partial r_{1}^{\nu}}\right)
$$

Considering the form of the tensor (246), we have

$$
K^{\mu \nu} v_{\perp}^{\mu}=\frac{\xi_{\perp}^{\nu}}{\xi^{2}}(\boldsymbol{\xi} . \mathbf{v})
$$


When substituted in Eq. (249), we see that the occurence of the $\delta$-function in the kinetic equation implies $E=0$. Finally, for the rate of entropy production we have, according to Eqs. (41) and (245):

$$
\dot{S}=\frac{N^{2} \gamma^{2}}{8} \int d^{2} \mathbf{r} d^{2} \mathbf{r}_{1} \frac{1}{P P_{1}} P_{1} \frac{\partial P}{\partial r^{\mu}} K^{\mu \nu} \delta(\boldsymbol{\xi} \cdot \mathbf{v})\left(P_{1} \frac{\partial P}{\partial r^{\nu}}-P \frac{\partial P_{1}}{\partial r_{1}^{\nu}}\right) .
$$

Permutting the dummy variables $\mathbf{r}$ and $\mathbf{r}_{1}$ and adding the resulting expression to Eq. (251), we obtain

$$
\dot{S}=\frac{N^{2} \gamma^{2}}{16} \int d^{2} \mathbf{r} d^{2} \mathbf{r}_{1} \frac{1}{P P_{1}} \delta(\boldsymbol{\xi} \cdot \mathbf{v})\left(P_{1} \frac{\partial P}{\partial r^{\mu}}-P \frac{\partial P_{1}}{\partial r_{1}^{\mu}}\right) K^{\mu \nu}\left(P_{1} \frac{\partial P}{\partial r^{\nu}}-P \frac{\partial P_{1}}{\partial r_{1}^{\nu}}\right) .
$$

Now, for any vector, $A^{\mu} K^{\mu \nu} A^{\nu}=\left(\mathbf{A} \cdot \boldsymbol{\xi}_{\perp}\right)^{2} / \xi^{2} \geq 0$. This proves a H-theorem $(\dot{S} \geq 0)$ for the kinetic equation (245). It should be emphasized that the conservation laws and the H-theorem result essentially from the symmetry of the kinetic equation. This is satisfying from a physical point of view.

It is also easy to show that the Boltzmann distribution

$$
P=A e^{-\beta \gamma\left(\psi+\frac{\Omega}{2} r^{2}-\mathbf{U}_{\perp} \mathbf{r}\right)},
$$

is a stationary solution of Eq. 245). Noting that

$$
\frac{\partial P}{\partial r^{\nu}}=-\beta \gamma\left(\frac{\partial \psi}{\partial r^{\nu}}+\Omega r^{\nu}-U_{\perp}^{\nu}\right) P
$$

we have successively

$$
K^{\mu \nu}\left(P_{1} \frac{\partial P}{\partial r^{\nu}}-P \frac{\partial P_{1}}{\partial r_{1}^{\nu}}\right)=\beta \gamma P P_{1} K^{\mu \nu}\left(v_{\perp}^{\nu}+\Omega \xi^{\nu}\right)=\beta \gamma P P_{1} \frac{\xi_{\perp}^{\mu}}{\xi^{2}}(\boldsymbol{\xi} . \mathbf{v}),
$$

where we have used Eqs. (248) and (250). When Eq. (255) is substituted in Eq. 245), we find that the right hand side cancels out due to the $\delta$-function. The advective term is also zero since $P=f\left(\psi^{\prime}\right)$. Therefore, the Boltzmann distribution (253) is a stationary solution of Eq. (245). Note, however, that this is not the only solution, unlike for ordinary kinetic equations. Any stationary solution of the Euler equation satisfying in addition $\boldsymbol{\xi} \cdot \mathbf{v} \neq 0$ for any couple of points $\mathbf{r}, \mathbf{r}_{1}$ (with $\mathbf{r} \neq \mathbf{r}_{1}$ ) is a solution of Eq. (245). Physically, this implies that the system needs sufficiently strong resonances to relax towards the maximum entropy state. If this condition is not realized, the system can remain frozen in a sort of "metastable" equilibrium state. Further evolution of the system will require non trivial correlations between point vortices which are not taken into account in the present theory.

\subsection{The thermal bath approximation}

A direct connexion between the generalized kinetic equation (245) and the Fokker-Planck equation of Sec. 1 can be found. Introducing a diffusion tensor

$$
D^{\mu \nu}=\frac{N \gamma^{2}}{8} \int d^{2} \mathbf{r}_{1} K^{\mu \nu} \delta(\boldsymbol{\xi} \cdot \mathbf{v}) P_{1}
$$


and a drift term

$$
\eta^{\mu}=-\frac{N \gamma^{2}}{8} \int d^{2} \mathbf{r}_{1} K^{\mu \nu} \delta(\boldsymbol{\xi} . \mathbf{v}) \frac{\partial P_{1}}{\partial r_{1}^{\nu}},
$$

we can rewrite Eq. 245) in the more illuminating form

$$
\frac{\partial P}{\partial t}+\langle\mathbf{V}\rangle \nabla P=\frac{\partial}{\partial r^{\mu}}\left[D^{\mu \nu} \frac{\partial P}{\partial r^{\nu}}+P \eta^{\mu}\right],
$$

similar to a general Fokker-Planck equation. Note, however, that Eq. (258) is an integrodifferential equation since the density probability $P(\mathbf{r}, t)$ in $\mathbf{r}$ at time $t$ depends on the value of the whole distribution of probability $P\left(\mathbf{r}_{1}, t\right)$ at the same time by an integration over $\mathbf{r}_{1}$. By contrast, the Fokker-Planck equation (182) is a differential equation. The usual way to transform an integrodifferential equation into a differential equation is to make a guess for the function $P\left(\mathbf{r}_{1}\right)$ appearing under the integral sign and refine the guess by successive iterations. In practice we simply make one sensible guess. Therefore, if we are close to equilibrium, it seems natural to replace the function $P_{1}$ appearing in the integrals by the Boltzmann distribution

$$
P\left(\mathbf{r}_{1}\right)=A e^{-\beta \gamma \psi\left(\mathbf{r}_{1}\right)} .
$$

This corresponds to the "thermal bath approximation" of Sec. 4: the vortices have not yet relaxed completely, but when we focus on the relaxation of a given point vortex (described by $P$ ) we can consider, in a first approximation, that the rest of the system (described by $P_{1}$ ) is at equilibrium. Within this approximation, the diffusion coefficient and the drift simplify in

$$
\begin{gathered}
\eta^{\mu}=\beta \gamma D^{\mu \nu} \frac{\partial \psi}{\partial r^{\nu}} \\
D^{\mu \nu}=\frac{N \gamma^{2}}{8} P(\mathbf{r}, t) \int K^{\mu \nu} \delta(\boldsymbol{\xi} . \mathbf{v}) d^{2} \boldsymbol{\xi}
\end{gathered}
$$

where we have made the local approximation. If we assume that the correlation time is short, i.e. if we replace $\xi^{2} \delta(\boldsymbol{\xi} . \mathbf{v})$ by $\tau / \pi^{2}$ (compare Eqs. (245) and (238)), we obtain

$$
\begin{gathered}
\boldsymbol{\eta}=\beta \gamma D \nabla \psi, \\
D=\frac{\gamma \tau}{16 \pi} \ln N\langle\omega\rangle,
\end{gathered}
$$

and Eq. 258) reduces to the Fokker-Planck equation

$$
\frac{\partial P}{\partial t}+\langle\mathbf{V}\rangle \nabla P=\nabla(D(\nabla P+\beta \gamma P \nabla \psi)) .
$$

This approximation is, however, not very satisfactory since the decorrelation time $\tau$ appears as a free parameter. In fact, the decorrelation time can be determined self-consistently from the above formulae by evaluating properly the $\delta$-function in Eq. (261). Expanding the velocity difference $\mathbf{v}=\left\langle\mathbf{V}_{1}\right\rangle-\langle\mathbf{V}\rangle$ in a Taylor series in $\boldsymbol{\xi}=\mathbf{r}_{1}-\mathbf{r}$, we obtain to first order in the expansion

$$
\xi . \mathbf{v}=\Sigma^{\mu \nu} \xi^{\mu} \xi^{\nu}
$$


where

$$
\Sigma^{\mu \nu}=\frac{1}{2}\left(\frac{\partial\langle V\rangle^{\mu}}{\partial r^{\nu}}+\frac{\partial\langle V\rangle^{\nu}}{\partial r^{\mu}}\right)
$$

is the stress tensor. It has the property of symmetry $\Sigma^{\mu \nu}=\Sigma^{\nu \mu}$. Since the flow is divergenceless, we also have $\Sigma^{x x}+\Sigma^{y y}=0$. In terms of the stress tensor (266), the diffusion tensor (261) can be rewritten

$$
D^{\mu \nu}=\frac{N P \gamma^{2}}{8} \int \frac{\xi^{2} \delta^{\mu \nu}-\xi^{\mu} \xi^{\nu}}{\xi^{2}} \delta\left(\Sigma^{\mu \nu} \xi^{\mu} \xi^{\nu}\right) d^{2} \boldsymbol{\xi}
$$

This integral can be performed easily by working in a basis $\left(\xi_{1}^{\prime}, \xi_{2}^{\prime}\right)$ in which the tensor $\Sigma^{\mu \nu}$ is anti-diagonal [36]. Then,

$$
D^{\mu \nu}=\frac{N P \gamma^{2}}{8} \int \frac{\xi^{\prime 2} \delta^{\mu \nu}-\xi^{\prime \mu} \xi^{\prime \nu}}{\xi^{\prime 2}} \delta\left(|\Sigma(\mathbf{r})| \xi_{1}^{\prime} \xi_{2}^{\prime}\right) d \xi_{1}^{\prime} d \xi_{2}^{\prime},
$$

where we have set $|\Sigma(\mathbf{r})|=2 \sqrt{-\operatorname{det}(\Sigma)}$. Clearly, this quantity is invariant by a change of reference frame and it measures the local shear of the flow. It is easy to check that the diffusion is isotropic and that $D^{\mu \nu}=D \delta^{\mu \nu}$ with

$$
D=\frac{N P \gamma^{2}}{8} \frac{1}{|\Sigma(\mathbf{r})|} \int \frac{\xi_{2}^{\prime 2}}{\xi_{1}^{\prime 2}+\xi_{2}^{\prime 2}} \delta\left(\xi_{1}^{\prime} \xi_{2}^{\prime}\right) d \xi_{1}^{\prime} d \xi_{2}^{\prime}
$$

Setting $\xi_{1}^{\prime}=\xi \cos \theta$ and $\xi_{2}^{\prime}=\xi \sin \theta$ where $\xi=\xi^{\prime}=\left|\mathbf{r}_{1}-\mathbf{r}\right|$, we obtain

$$
D=\frac{N P \gamma^{2}}{8} \frac{1}{|\Sigma(\mathbf{r})|} \int_{0}^{+\infty} \xi d \xi \int_{0}^{2 \pi} d \theta \sin ^{2} \theta \delta\left(\xi^{2} \cos \theta \sin \theta\right),
$$

or, equivalently,

$$
D=\frac{N P \gamma^{2}}{4} \frac{1}{|\Sigma(\mathbf{r})|} \int_{0}^{+\infty} \frac{d \xi}{\xi} \int_{0}^{\pi} d \theta \sin \theta \delta(\cos \theta)
$$

As explained previously, we regularize the logarithmic divergence by introducing appropriate cut-offs at small and large scales. With the change of variables $t=$ $\cos \theta$, we finally obtain

$$
D=\frac{N P \gamma^{2}}{8} \frac{1}{|\Sigma(\mathbf{r})|} \ln N \int_{-1}^{+1} d t \delta(t)=\frac{N P \gamma^{2}}{8} \frac{1}{|\Sigma(\mathbf{r})|} \ln N,
$$

which establishes Eq. (189) in the general case.

\subsection{The collisional relaxation time}

We can deduce from this kinetic theory the "collisional" relaxation time of the point vortex gas. Considering the Fokker-Planck equation (264), it is easy to check that, for $t \rightarrow+\infty$, the distribution function $P(\mathbf{r}, t)$ will converge towards the Boltzmann distribution (44). The relaxation time corresponds typically to 
the time needed by a vortex to diffuse over a distance $R$, the system size. Therefore, $t_{\text {relax }} \sim R^{2} / D$, with $D \sim \gamma \ln N$ according to Eq. (272). Using $\Gamma=N \gamma$ and introducing the dynamical time $t_{D} \sim\langle\omega\rangle^{-1} \sim R^{2} / \Gamma$, we obtain the estimate

$$
t_{\text {relax }} \sim \frac{N}{\ln N} t_{D},
$$

as in the case of collisional stellar systems [9]. Since the statistical description is expected to yield relevant results for large $N$, we conclude that the "collisional" relaxation of point vortices towards the Boltzmann distribution (44) is a very slow process. It can certainly not account for most of the numerical simulations and experiments of 2D turbulence and point vortex dynamics in which an equilibrium state is established extremely rapidly. This implies that a more violent relaxation mechanism must be at work in the system (see Sec. 6). This is a remark of crucial importance because it means that all the results established previously, including the Boltzmann distribution (44), must be revised.

\section{Violent relaxation of $2 \mathrm{D}$ vortices and stellar systems}

\subsection{The Euler and the Vlasov equations}

In the preceding sections, we have focused our attention to the point vortex model as an idealization of more realistic flows which necessarily involve a continuous vorticity distribution. This approximation is interesting in a first approach because it leads to a system of $N$ particles in interaction (like electric charges or stars) for which the methods of statistical mechanics are directly applicable. In addition, this model keeps the specificity of two-dimensional vorticity flows such as long-range interactions between vortices and structure formation. However, there are many different ways to approximate a continuous vorticity field by a cloud of point vortices and different approximations can lead to different statistical equilibrium states (this difficulty was underlined by Onsager [101]). Therefore, if we want to apply the results of statistical mechanics to realistic situations (e.g., geophysical flows) it is necessary to go beyond the point vortex model and develop a statistical mechanics for continuous vorticity fields.

For flows of geophysical or astrophysical interest, the Reynolds numbers are so high that the molecular viscosity is not expected to play a crucial role in the dynamics. Therefore, these flows are described in the simplest model by the Euler-Poisson system

$$
\begin{gathered}
\frac{\partial \omega}{\partial t}+\mathbf{u} \nabla \omega=0, \\
\omega=-\Delta \psi .
\end{gathered}
$$

It can be recalled that these equations also model the early dynamics of a cloud of point vortices before correlations between vortices have developed (in that case, $\omega$ is proportional to the one-body distribution function $P(\mathbf{r}, t)$, see Eq. (233)). As discussed in Sec. 5.9, this is the regime of physical interest since the "collisional" relaxation of point vortices is in general very slow. 
Similarly, for a majority of stellar systems, including the important class of elliptical galaxies, the relaxation time by two-body encounters is $\sim 10^{12}$ times larger than the age of the universe. Therefore, the dynamics of stars in a galaxy is essentially collisionless and appropriately described by the Vlasov-Poisson system

$$
\begin{gathered}
\frac{\partial f}{\partial t}+\mathbf{v} \frac{\partial f}{\partial \mathbf{r}}+\mathbf{F} \frac{\partial f}{\partial \mathbf{v}}=0 \\
\Delta \Phi=4 \pi G \int f d^{3} \mathbf{v} .
\end{gathered}
$$

The morphological similarity of the Euler-Poisson and Vlasov-Poisson systems is another manifestation of the close analogy between $2 \mathrm{D}$ vortices and stellar systems. If we make the correspondance between the vorticity and the distribution function $(\omega \leftrightarrow f)$ and between the stream function and the gravitational potential $(\psi \leftrightarrow \Phi)$, these two equations describe the advection of a density by an incompressible flow with which it interacts via a Poisson equation. Then, the density is not advected passively by the flow but is coupled to its motion. This coupling is responsible for violent fluctuations of the stream function or gravitational potential. These fluctuations will mix the vorticity or the phase elements at small scales and induce a self-organization and the appearance of structures at larger scales (see Fig. 13). This violently changing potential provides a mechanism analogous to a relaxation in a gas, but the specificity of this relaxation is that it is collisionless and due to the long-range nature of the interactions. It is now clear that this "chaotic mixing" is the driving source of relaxation in two-dimensional turbulence and stellar dynamics. The kinetic theory presented in Sec. 5 for point vortices (and the one developed by Chandrasekhar for stars) is only valid in situations in which this chaotic mixing is prevented or has died away.

\subsection{The statistical equilibrium}

During the mixing process, the Vlasov-Poisson and the Euler-Poisson systems generate intermingled filaments at smaller and smaller scales. Therefore, a deterministic description of the flow would require a rapidly increasing amount of information as time goes on. For that reason, it is appropriate to undertake a probabilistic description in order to smooth out the small scales and concentrate on the locally averaged quantities. This statistical approach, called the theory of "violent relaxation", was introduced by Lynden-Bell [90] in 1967 for collisionless stellar systems and rediscovered independantly by Kuzmin [83, Miller [95, 96. and Robert \& Sommeria [11] for two-dimensional vorticity flows. The analogy between these two statistical mechanics (including the relaxation towards equilibrium) was discussed in detail by Chavanis [29,54,32, 35. On the other hand, a rigourous justification of this statistical approach has been given by Robert [109 by using the concept of Young measures and large deviations. This theory improves upon previous works based on a spectral representation of the flow [80, which do not respect all the conservation laws of the invisicd dynamics. In 
$y \uparrow$
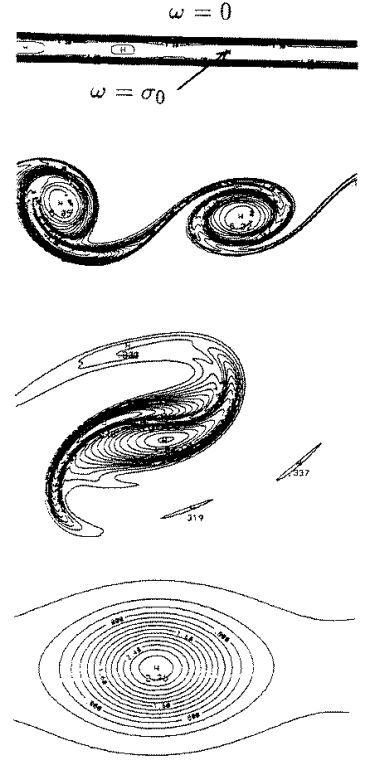

$v \uparrow$
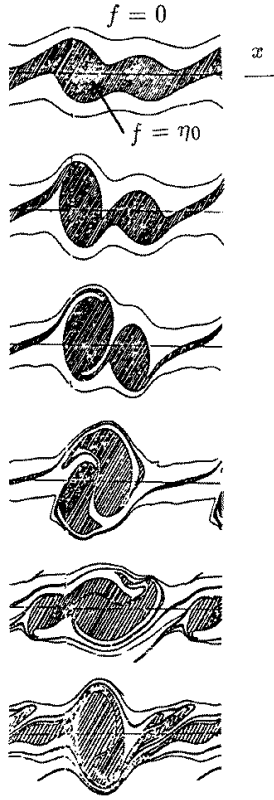

Fig. 13. Violent relaxation of two-dimensional vorticity flows and self-gravitating systems. The left panel corresponds to the nonlinear developement of the KelvinHelmholtz instability. The equilibrium state is a large-scale vortex which is welldescribed by statistical mechanics [119]. The right panel corresponds to a simulation of the Vlasov-Poisson system showing a mixing process and the formation of a coherent structure in phase space with a Fermi-Dirac distribution [7]. This simulation is restricted to a one-dimensional system but the process remains the same in higher dimensions.

the following, we present the statistical mechanics of violent relaxation for twodimensional vorticity flows. A discussion of the statistical mechanics of violent relaxation in stellar systems, closely following the presentation of this paper, can be found in 42 .

In the statistical approach, the exact knowledge of the "fine-grained" or microscopic vorticity field is replaced by the probability density $\rho(\mathbf{r}, \sigma)$ of finding the vorticity level $\sigma$ in $\mathbf{r}$. The normalization condition yields at each point

$$
\int \rho(\mathbf{r}, \sigma) d \sigma=1
$$

and the locally averaged (coarse-grained) vorticity is expressed in terms of the density probability in the form

$$
\bar{\omega}=\int \rho(\mathbf{r}, \sigma) \sigma d \sigma .
$$

More generally, the moments of the vorticity are defined by

$$
\overline{\omega^{n}}=\int \rho(\mathbf{r}, \sigma) \sigma^{n} d \sigma .
$$


During the evolution, the energy

$$
E=\frac{1}{2} \int \bar{\omega} \psi d^{2} \mathbf{r}
$$

is conserved as well as the total area of each level of vorticity

$$
\gamma(\sigma)=\int \rho(\mathbf{r}, \sigma) d^{2} \mathbf{r}
$$

These last constraints are equivalent to the conservation of the Casimir integrals $C_{h}=\int h(\omega) d^{2} \mathbf{r}$ for any continuous function $h$. Such integrals are conserved by the Euler equation because the fluid particles keep their vorticity (on account of the transport equation $d \omega / d t=0$ ) and their surface (on account of the incompressibility of the flow).

After a complex evolution the system is expected to be in the most probable, i.e. most mixed state, consistent with all the constraints imposed by the dynamics. We define the mixing entropy as the logarithm of the number of microscopic configurations associated with the same macroscopic state (characterized by the probability density $\rho(\mathbf{r}, \sigma))$. We divide the macrocells $(\mathbf{r}, \mathbf{r}+d \mathbf{r})$ into $\nu$ microcells and denote by $n_{i j}$ the number of microcells occupied by the vorticity level $\sigma_{j}$ in the $i$-th macrocell. A simple combinatorial argument indicates that the number of microstates associated with the macrostate $\left\{n_{i j}\right\}$ is

$$
W\left(\left\{n_{i j}\right\}\right)=\prod_{i} N_{j} ! \prod_{i} \frac{\nu !}{n_{i j} !}
$$

where $N_{j}=\sum_{i} n_{i j}$ is the total number of microcells occupied by $\sigma_{j}$. We have to add the normalization condition $\sum_{j} n_{i j}=\nu$, equivalent to Eq. (278), which prevents overlapping of different vorticity levels. This constraint plays a role similar to the Pauli exclusion principle in quantum mechanics. Morphologically, the statistics (283) corresponds to a $4^{\text {th }}$ type of statistics since the particles are distinguishable but subject to an exclusion principle 90. There is no such exclusion for point vortices (in the collisional regime) since they are free a priori to approach each other without limitation.

Taking the logarithm of $W$ and passing to the continuum limit with the aid of the Stirling formula, we get

$$
S=-\int \rho(\mathbf{r}, \sigma) \ln \rho(\mathbf{r}, \sigma) d^{2} \mathbf{r} d \sigma
$$

The most probable macroscopic state is obtained by maximizing the mixing entropy (284) with fixed energy (281), global vorticity distribution (282) and local normalization (278). This problem is treated by introducing Lagrange multipliers, so that the first variations satisfy

$$
\delta S-\beta \delta E-\int \alpha(\sigma) \delta \gamma(\sigma) d \sigma-\int \zeta(\mathbf{r}) \delta\left(\int \rho(\mathbf{r}, \sigma) d \sigma\right) d^{2} \mathbf{r}=0
$$


The resulting optimal probability is a Gibbs state which can be expressed as

$$
\rho(\mathbf{r}, \sigma)=\frac{1}{Z(\psi)} g(\sigma) e^{-\beta \sigma \psi}
$$

where $Z(\psi) \equiv e^{\zeta(\mathbf{r})+1}$ and $g(\sigma) \equiv e^{-\alpha(\sigma)}$. The normalization condition (278) leads to a value of the partition function $Z$ of the form

$$
Z=\int g(\sigma) e^{-\beta \sigma \psi} d \sigma
$$

and the locally averaged vorticity $(279)$ is expressed as a function of $\psi$ according to

$$
\bar{\omega}=\frac{\int g(\sigma) \sigma e^{-\beta \sigma \psi} d \sigma}{\int g(\sigma) e^{-\beta \sigma \psi} d \sigma}=f(\psi)
$$

This expression can be rewritten

$$
\bar{\omega}=-\frac{1}{\beta} \frac{d \ln Z}{d \psi} .
$$

Differentiating Eq. (288) with respect to $\psi$, we check that the variance of the vorticity can be written 53

$$
\omega_{2} \equiv \overline{\omega^{2}}-\bar{\omega}^{2}=-\frac{1}{\beta} f^{\prime}(\psi)
$$

or, alternatively,

$$
\omega_{2}=\frac{1}{\beta^{2}} \frac{d^{2} \ln Z}{d \psi^{2}} .
$$

Therefore, the slope of the function $\bar{\omega}=f(\psi)$ is directly related to the variance of the vorticity distribution. Since $\omega_{2}>0$, we find that the function $\bar{\omega}=f(\psi)$ is monotonic; it is decreasing for $\beta>0$ and increasing for $\beta<0$ (it is constant for $\beta=0$ ). Another proof of this result is given in 111.

Two particular cases are worth considering. If the local distribution of vorticity is Gaussian, then the $\omega-\psi$ relationship (288) is linear 959.96. On the other hand, in the case of a single level of vorticity $\sigma_{0}$ (in addition to the level $\sigma=0)$, the coarse-grained vorticity $\omega=\rho\left(\mathbf{r}, \sigma_{0}\right) \sigma_{0}$ takes explicitly the form

$$
\bar{\omega}=\frac{\sigma_{0}}{1+\lambda e^{\beta \sigma_{0} \psi}} .
$$

This is formally similar to the Fermi-Dirac statistics. Here, the exclusion principle $\bar{\omega} \leq \sigma_{0}$ is due to the Liouville theorem (i.e., the conservation of the finegrained vorticity) not to quantum mechanics. Because of the averaging procedure, the coarse-grained vorticity can only decrease by internal mixing, as irrotational flow is incorporated into the patch $\sigma_{0}$, and this results in an "effective" exclusion principle. In the dilute limit $\bar{\omega} \ll \sigma_{0}$, Eq. (292) reduces to the Boltzmann distribution $\bar{\omega}=A e^{-\beta \sigma_{0} \psi}$ as in the point vortex model [70,107. 
Similar results are obtained in the context of collisionless stellar system described by the Vlasov-Poisson system 90,42. The equivalent of Eq. (292) is the Lynden-Bell distribution function

$$
\bar{f}=\frac{\eta_{0}}{1+\lambda e^{\beta \eta_{0}\left(\frac{v^{2}}{2}+\Phi\right)}},
$$

which formally coincides with the distribution function of the self-gravitating Fermi gas. In the non-degenerate limit $\bar{f} \ll \eta_{0}$, it reduces to the MaxwellBoltzmann distribution $\bar{f}=A e^{-\beta \eta_{0}\left(\frac{v^{2}}{2}+\Phi\right)}$. Therefore, the theory of violent relaxation naturally explains the observed isothermal cores of elliptical galaxies without recourse to collisions [90,63.

\subsection{The Maximum Entropy Production Principle}

Basically, a two-dimensional incompressible turbulent flow at high Reynolds numbers is described by the Euler-Poisson system. In principle, these equations determine completely the evolution of the vorticity field $\omega(\mathbf{r}, t)$. However, in practice, we are not interested by the finely striated structure of the flow but only by its smoothed-out structure. Indeed, the observations and the numerical simulations are always realized with a finite resolution. Moreover, the coarsegrained vorticity $\bar{\omega}$ is expected to converge towards an equilibrium state, the Gibbs state (286), contrary to the exact vorticity field $\omega$ which develops smaller and smaller scales. There is also a technical difficulty to simulate an inviscid dynamics due precisely to the developement of this small-scale motion. Contour dynamics methods need to introduce a "surgery" and spectral codes a "viscosity" in order to prevent numerical instabilities. However, this artificial viscosity breaks the conservation laws of the Euler equations. What we would like to obtain is a set of relaxation equations which smooth out the small scales while conserving all the constraints of the Euler equation. Such a parametrization has been proposed by Robert \& Sommeria 112 in terms of a phenomenological Maximum Entropy Production Principle (MEPP).

Let us decompose the vorticity $\omega$ and velocity $\mathbf{u}$ into a mean and a fluctuating part, namely $\omega=\bar{\omega}+\tilde{\omega}, \mathbf{u}=\overline{\mathbf{u}}+\tilde{\mathbf{u}}$. Taking the local average of the Euler equation (274), we get

$$
\frac{\partial \bar{\omega}}{\partial t}+\nabla(\bar{\omega} \overline{\mathbf{u}})=-\nabla \mathbf{J}_{\omega}
$$

where the current $\mathbf{J}_{\omega}=\overline{\tilde{\omega} \tilde{\mathbf{u}}}$ represents the correlations of the fine-grained fluctuations. Eq. (294) can be viewed as a local conservation law for the circulation $\Gamma=\int \bar{\omega} d^{2} \mathbf{r}$. To apply the MEPP, we need to consider not only the locally averaged vorticity field $\bar{\omega}$ but the whole probability distribution $\rho(\mathbf{r}, \sigma, t)$ now evolving with time $t$. The conservation of the global vorticity distribution $\gamma(\sigma)=\int \rho d^{2} \mathbf{r}$ can be written in the local form

$$
\frac{\partial \rho}{\partial t}+\nabla(\rho \mathbf{u})=-\nabla \mathbf{J}
$$


where $\mathbf{J}(\mathbf{r}, \sigma, \mathbf{t})$ is the (unknown) current associated with the vorticity level $\sigma$. Integrating Eq. (295) over all the vorticity levels $\sigma$, using Eq. (278), and comparing with Eq. (3), we find the constraint

$$
\int \mathbf{J}(\mathbf{r}, \sigma, t) d \sigma=\mathbf{0}
$$

Multiplying Eq. 295) by $\sigma$, integrating over all the vorticity levels, using Eq. (279), and comparing with Eq. (294), we get $\int \mathbf{J}(\mathbf{r}, \sigma, t) \sigma d \sigma=\mathbf{J}_{\omega}$.

We can express the time variation of energy in terms of $\mathbf{J}$, using Eqs. (281) and (294), leading to the energy conservation constraint

$$
\dot{E}=\int \mathbf{J}_{\omega} \nabla \psi d^{2} \mathbf{r}=0
$$

Using Eqs. (284) and (295), we similarly express the rate of entropy production as

$$
\dot{S}=-\int \mathbf{J} \nabla(\ln \rho) d^{2} \mathbf{r} d \sigma .
$$

The Maximum Entropy Production Principle (MEPP) consists in choosing the current $\mathbf{J}$ which maximizes the rate of entropy production $\dot{S}$ respecting the constraints $\dot{E}=0, \int \mathbf{J} d \sigma=\mathbf{0}$ and $\int \frac{J^{2}}{2 \rho} d \sigma \leq C(\mathbf{r}, t)$. The last constraint expresses a bound (unknown) on the value of the diffusion current. Convexity arguments justify that this bound is always reached so that the inequality can be replaced by an equality. The corresponding condition on first variations can be written at each time $t$ :

$$
\delta \dot{S}-\beta(t) \delta \dot{E}-\int \boldsymbol{\zeta}(\mathbf{r}, t) \delta\left(\int \mathbf{J} d \sigma\right) d^{2} \mathbf{r}-\int D^{-1}(\mathbf{r}, t) \delta\left(\int \frac{J^{2}}{2 \rho} d \sigma\right) d^{2} \mathbf{r}=0,
$$

and leads to a current of the form

$$
\mathbf{J}=-D(\mathbf{r}, t)[\nabla \rho+\beta(t) \rho(\sigma-\bar{\omega}) \nabla \psi] .
$$

The Lagrange multiplier $\boldsymbol{\zeta}$ has been eliminated, using the condition (296) of local normalization conservation. The conservation of energy (297) at any time determines the evolution of the Lagrange multiplier $\beta(t)$ according to

$$
\beta(t)=-\frac{\int D \nabla \bar{\omega} \nabla \psi d^{2} \mathbf{r}}{\int D \omega_{2}(\nabla \psi)^{2} d^{2} \mathbf{r}}
$$

The entropy production (298) can be written

$$
\dot{S}=-\int \frac{\mathbf{J}}{\rho}(\nabla \rho+\beta \rho(\sigma-\bar{\omega}) \nabla \psi) d^{2} \mathbf{r} d \sigma+\beta \int \mathbf{J}(\sigma-\bar{\omega}) \nabla \psi d^{2} \mathbf{r} d \sigma .
$$


Using Eqs. (296) and (297), the second integral is seen to cancel out. Inserting Eq. (300) in the first integral, we find

$$
\dot{S}=\int \frac{J^{2}}{D \rho} d^{2} \mathbf{r} d \sigma
$$

which is positive provided that $D \geq 0$. A stationary solution $\dot{S}=0$ is such that $\mathbf{J}=\mathbf{0}$ yielding, together with Eq. (300),

$$
\nabla(\ln \rho)+\beta(\sigma-\bar{\omega}) \nabla \psi=\mathbf{0} .
$$

For any reference vorticity level $\sigma_{0}$, it writes

$$
\nabla\left(\ln \rho_{0}\right)+\beta\left(\sigma_{0}-\bar{\omega}\right) \nabla \psi=\mathbf{0} .
$$

Substracting Eqs. (304) and (305), we obtain $\nabla \ln \left(\rho / \rho_{0}\right)+\beta\left(\sigma-\sigma_{0}\right) \nabla \psi=\mathbf{0}$, which is immediately integrated into

$$
\rho(\mathbf{r}, \sigma)=\frac{1}{Z(\mathbf{r})} g(\sigma) e^{-\beta \sigma \psi},
$$

where $Z^{-1}(\mathbf{r}) \equiv \rho_{0}(\mathbf{r}) e^{\beta \sigma_{0} \psi(\mathbf{r})}$ and $g(\sigma) \equiv e^{A(\sigma)}, A(\sigma)$ being a constant of integration. Therefore, entropy increases until the Gibbs state (286) is reached, with $\beta=\lim _{t \rightarrow \infty} \beta(t)$. Furthermore, we can show that a stationary solution of these relaxation equations is linearly stable if, and only if, it is an entropy maximum (in preparation). Therefore, this numerical algorithm selects the maxima (and not the minima or the saddle points) among all critical points of entropy. When several entropy maxima subsist for the same values of the constraints, the choice of equilibrium depends on a complicated notion of "basin of attraction" and not simply whether the solution is a local or a global entropy maximum (see Ref. [46] in a related context).

The relaxation equations (295), (300) and (301) can be simplified in the single level approximation. In that case, Eq. (294) is explicitly given by

$$
\frac{\partial \bar{\omega}}{\partial t}+\overline{\mathbf{u}} \nabla \bar{\omega}=\nabla\left[D\left(\nabla \bar{\omega}+\beta(t) \bar{\omega}\left(\sigma_{0}-\bar{\omega}\right) \nabla \psi\right)\right] .
$$

In the dilute limit $\bar{\omega} \ll \sigma_{0}$, it takes a form similar to the Fokker-Planck equation (206) obtained for point vortices. These equations both involve a diffusion and a drift, but these terms have a different physical interpretation in each case.

It is also instructive to apply this thermodynamical approach to the VlasovPoisson system. In the single level approximation, it leads to the following equation for the coarse-grained distribution function [54,31]:

$$
\frac{\partial \bar{f}}{\partial t}+\mathbf{v} \frac{\partial \bar{f}}{\partial \mathbf{r}}+\mathbf{F} \frac{\partial \bar{f}}{\partial \mathbf{v}}=\frac{\partial}{\partial \mathbf{v}}\left[D\left(\frac{\partial \bar{f}}{\partial \mathbf{v}}+\beta(t) \bar{f}\left(\eta_{0}-\bar{f}\right) \mathbf{v}\right)\right],
$$

which is a generalized form of the familiar Fokker-Planck equation (208) recovered in the non degenerate limit $\bar{f} \ll \eta_{0}$. We can check that Eq. (308) returns the Lynden-Bell distribution function (293) at equilibrium. 
The diffusion coefficient $D$ is not determined by the MEPP as it depends on the unknown bound $C$ on the current. For the purpose of reaching the Gibbs state (286), the diffusion coefficient can simply be chosen arbitrarily (but with a positive value in order to ensure entropy increase). However, the precise form of the diffusion coefficient is important in order to determine the relaxation time and take into account kinetic confinement and incomplete relaxation (see below). In the context of 2D turbulence, Robert \& Rosier [110] have proposed a simple evaluation of $D$ by using an analogy with the diffusion of a passive scalar $\omega$ subjected to a turbulent velocity field $\mathbf{u}=\overline{\mathbf{u}}+\tilde{\mathbf{u}}$ (see also [54, Appendix B). In that case, the mean value $\bar{\omega}$ satisfies a convection-diffusion equation

$$
\frac{\partial \bar{\omega}}{\partial t}+(\mathbf{u} \nabla) \bar{\omega}=\nabla(D \nabla \bar{\omega})
$$

with a diffusion coefficient given by

$$
D=\frac{1}{4} \tau \overline{\tilde{\mathbf{u}}^{2}}(\mathbf{r}, t)
$$

where $\tau$ is the decorrelation time of the velocity fluctuations. Equation (307) reduces to Eq. (310) when $\beta=0$, i.e. when the energy constraint is not active. In the Euler-Poisson system, the velocity is produced by the vorticity itself via the Biot \& Savart formula

$$
\mathbf{u}(\mathbf{r}, t)=\int \omega\left(\mathbf{r}^{\prime}, t\right) \mathbf{V}\left(\mathbf{r}^{\prime} \rightarrow \mathbf{r}\right) d^{2} \mathbf{r}^{\prime},
$$

where $\mathbf{V}\left(\mathbf{r}^{\prime} \rightarrow \mathbf{r}\right)=-(1 / 2 \pi) \mathbf{z} \times\left(\mathbf{r}^{\prime}-\mathbf{r}\right) /\left|\mathbf{r}^{\prime}-\mathbf{r}\right|^{2}$. Inserting Eq. (311) in Eq. (310), we obtain

$$
D=\frac{\tau}{4} \int \overline{\tilde{\omega}\left(\mathbf{r}^{\prime}, t\right) \tilde{\omega}\left(\mathbf{r}^{\prime \prime}, t\right)} \mathbf{V}\left(\mathbf{r}^{\prime} \rightarrow \mathbf{r}\right) \mathbf{V}\left(\mathbf{r}^{\prime \prime} \rightarrow \mathbf{r}\right) d^{2} \mathbf{r}^{\prime} d^{2} \mathbf{r}^{\prime \prime} .
$$

If we neglect the spatial correlations of the vorticity fluctuations on scales larger than $\epsilon$, the resolution scale, we have

$$
\overline{\tilde{\omega}\left(\mathbf{r}^{\prime}, t\right) \tilde{\omega}\left(\mathbf{r}^{\prime \prime}, t\right)}=\epsilon^{2} \overline{\tilde{\omega}^{2}}\left(\mathbf{r}^{\prime}, t\right) \delta\left(\mathbf{r}^{\prime}-\mathbf{r}^{\prime \prime}\right),
$$

and Eq. (312) reduces to

$$
D=\frac{\tau}{4} \epsilon^{2} \int \overline{\tilde{\omega}^{2}}\left(\mathbf{r}^{\prime}, t\right) \mathbf{V}^{2}\left(\mathbf{r}^{\prime} \rightarrow \mathbf{r}\right) d^{2} \mathbf{r}^{\prime} .
$$

Making a local approximation and introducing an upper cut-off $a$, we obtain

$$
D=\frac{\tau}{4} \epsilon^{2} \overline{\tilde{\omega}^{2}}(\mathbf{r}, t) \int_{\epsilon}^{a}\left(\frac{1}{2 \pi \xi}\right)^{2} 2 \pi \xi d \xi,
$$

with $\overline{\tilde{\omega}^{2}}=\overline{\omega^{2}}-\bar{\omega}^{2}$. This leads to the following expression for the diffusion coefficient

$$
D=\left(\overline{\omega^{2}}-\bar{\omega}^{2}\right) \frac{\tau \epsilon^{2}}{8 \pi} \ln \left(\frac{a}{\epsilon}\right) .
$$


We note that the diffusion coefficient vanishes in regions where there is no fluctuation of the vorticity at small scales, i.e. $\omega_{2}=0$. At the contact with the unmixed flow, the diffusion current also vanishes resulting in a confinement of the vorticity. This leads to the concept of incomplete relaxation: in a large-scale turbulent flow, the Gibbs state (286) is satisfied only in restricted regions of space where mixing is sufficiently efficient to justify an ergodic hypothesis. Outside these domains, the relaxation is slowed down or even stopped. This kinetic confinement is illustrated by the numerical simulations of Robert \& Rosier [110] and further discussed by Chavanis \& Sommeria [51]. In this viewpoint, the vortices of two-dimensional turbulence can be considered as restricted equilibrium states or maximum entropy bubbles [51] separated from each other by an almost irrotational background. A similar kinetic confinement can be advocated in the case of stellar systems [54,42 to account for incomplete relaxation [90] and solve the infinite mass problem.

\subsection{Recent developements}

An interesting problem in fluid dynamics is to obtain a classification of the "zoology" of vortices (monopoles, translating and rotating dipoles, tripoles...) met in two-dimensional flows. The statistical mechanics approach presented in Sec. 6.2 provides a general framework to tackle this problem as it selects the most probable structures among all possible solutions of the 2D Euler equations. However, the prediction is not straightforward because, in the general case, we have to take into account an infinite set of constraints, namely the conservation of all the Casimirs $C_{h}$, in addition to energy $E$, angular momentum $L$ and impulse $P$. A numerical algorithm has been developed by Turkington \& Whitaker [124] to solve this problem and several calculations have been performed in rectangular or circular domains for a finite number of vorticity levels and for particular values of the integral constraints. However, many structures are found and it is difficult to have a clear picture of the bifurcation diagram in parameter space. For that reason, Chavanis \& Sommeria 49,51] have considered a particular limit of the statistical theory, the so-called "strong mixing limit", in which the study of these bifurcations can be performed analytically. This limit corresponds to $\beta \sigma \psi \ll 1$ so that the equations of the problem can be expanded in terms of this small parameter (this is like the Debye-Hückel approximation in plasma physics). To zeroth order in the expansion, the density probability of each level is uniform which corresponds to a completely mixed state. To first order, the relationship between vorticity and streamfunction is linear and this can justify an inviscid minimum enstrophy principle (for the coarse-grained enstrophy $\Gamma_{2}^{c . g}=\int \bar{\omega}^{2} d^{2} \mathbf{r}$ ) [49. In that case, the equilibrium flow only depends on $E, L, P$ and the first moment $\Gamma$ of the vorticity (the fine-grained enstrophy $\Gamma_{2}^{f . g .}=\int \overline{\omega^{2}} d^{2} \mathbf{r}$ serves as a normalization factor). This particular limit of the statistical theory already exhibits a rich bifurcation diagram and often provides a good approximation of more general situations (in particular for weakly energetic flows). It is also possible, in principle, to go to higher orders in the expansion in which case more and more vorticity moments $\Gamma_{3}, \Gamma_{4}, \ldots$ are necessary to describe the structure 
of equilibrium. This limit makes therefore a hierarchy between the constraints as it shows that, in many situations, only the lowest moments of the vorticity are important to characterize the equilibrium state. Because of this hierarchy, we can make some relevant predictions without the complete knowledge of the initial condition.

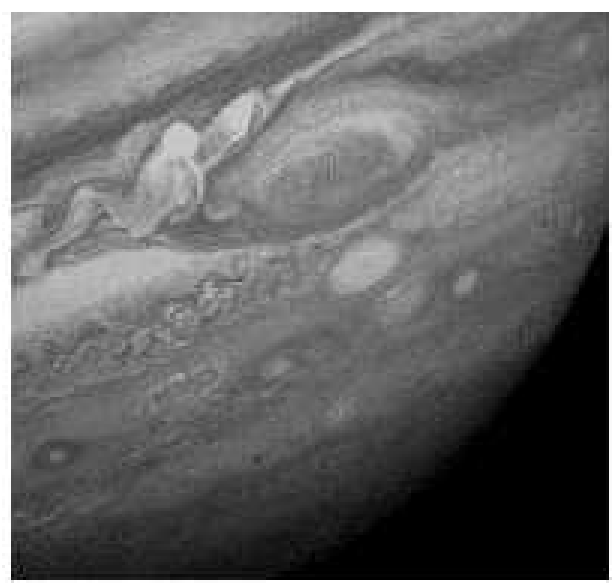

Fig. 14. Jupiter's great red spot

The statistical mechanics approach can be extended immediately to the quasi-geostrophic (QG) equations 94 by simply replacing the vorticity by the potential vorticity (PV). The formalism has also been generalized by Chavanis \& Sommeria [53] to the shallow-water (SW) equations. These equations are more relevant to describe geophysical flows than the 2D Euler equations [104]. In the QG approximation, Bouchet \& Sommeria 14 have explained the formation of jets and vortices in planetary atmospheres in terms of statistical mechanics, as initiated in [118]. In particular, the annular jet structure of Jupiter's Great Red Spot (see Figs. 14 15) is reproduced and explained as the coexistence of two thermodynamical phases in contact (a picture which is rigorously valid in a small Rossby radius expansion). These results can be extended to the more general case of shallow water equations [13]. In this geophysical context, the deformation of the fluid surface tends to reduce the range of interaction between vortices. This situation is comparable to what happens in a neutral plasma: the interaction between vortices is shielded on a distance of the order of the Rossby length, the analogous of the Debye length in plasma physics.

The relaxation equations presented in Sec. 6.3 provide a convenient parameterization of sub-grid scale eddies which drives the system toward statistical equilibrium by a continuous time evolution. Such relaxation equations can be used both as a realistic coarse resolution model of the turbulent evolution, and as a method of determination of the statistical equilibrium resulting from this evolution. However, these equations do not preserve the invariance properties of 

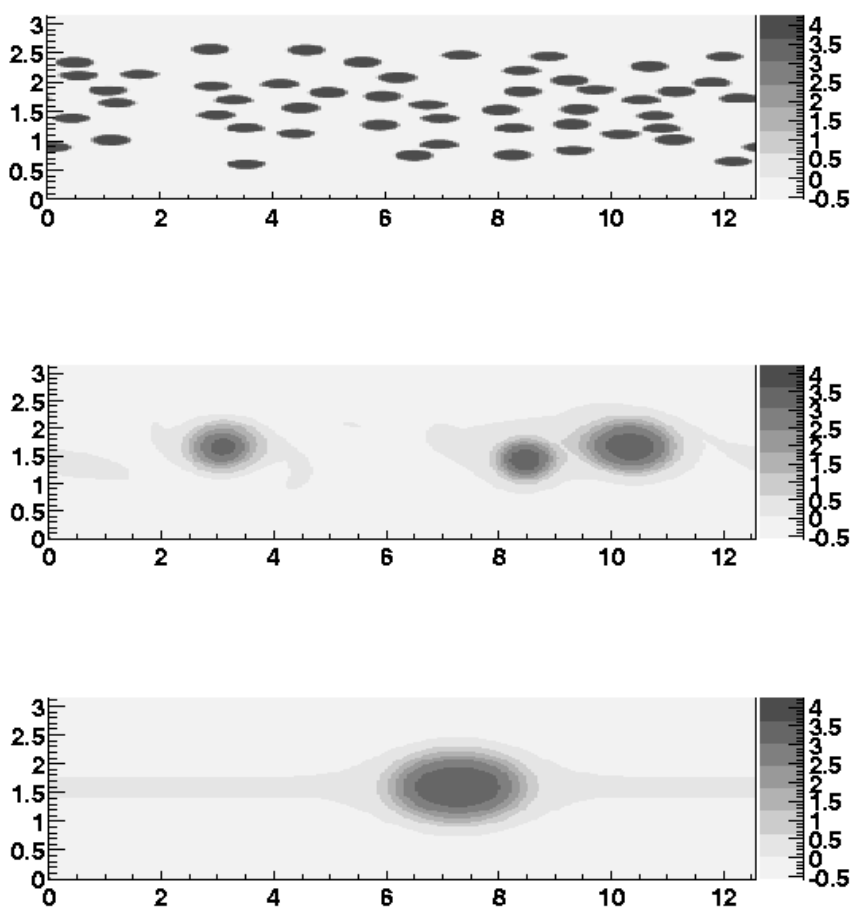

Fig. 15. Relaxation towards statistical equilibrium in a QG model of Jupiter's great red spot (from Ref. 12]). Three successive potential vorticity (PV) fields are represented as grey levels. The initial condition (top) is made of small PV patches. These patches organize into vortices (middle) that eventually merge into a single one (bottom). This sequence is obtained with the relaxation equations described in Sec. 6.3, applied to the Q.G. situation: entropy increases with time while energy is exactly conserved. At equilibrium, the vortex is an oval spot of quasiuniform PV surrounded by strong gradients, corresponding to an annular jet.

the Euler equations by a change of reference frame. In addition, the conservation of energy is enforced by a formal Lagrange multiplier $\beta(t)$ which is uniform in space. This may be a limitation to describe large-scale turbulent flows which organize locally in several types of structures with a different temperature. A generalization of the MEPP has been proposed by Chavanis \& Sommeria [50] as an attempt to solve these difficulties. More general, but also more complex, relaxation equations are obtained. They involve a space dependant temperature which tends to be uniform in each vortex but with an a priori different value from one vortex to the other. A simplified version of these relaxation equations has been solved numerically by Kazantzev et al. [78] in an oceanic context.

Despite its practical interest, the main drawback of the MEPP is its ad hoc nature. In [34], we have attempted to justify the relaxation equation (307) from first principles starting directly from the 2D Euler equation. A systematic derivation can be obtained in the so-called quasilinear approximation. This approximation is well-known in plasma physics and stellar dynamics 71, 114, 122 
for the Vlasov-Poisson system and we tried to extend it to the Euler-Poisson system. Substracting Eq. (274) and (294) and neglecting the non linear terms in the equation for $\tilde{\omega}$, the basic equations of the quasilinear theory are

$$
\begin{aligned}
& \frac{\partial \bar{\omega}}{\partial t}+\overline{\mathbf{u}} \nabla \bar{\omega}=-\nabla \overline{\tilde{\omega} \tilde{\mathbf{u}}} \\
& \frac{\partial \tilde{\omega}}{\partial t}+\overline{\mathbf{u}} \nabla \tilde{\omega}=-\tilde{\mathbf{u}} \nabla \bar{\omega} .
\end{aligned}
$$

It is possible to solve Eq. (318) formally with the aid of Green's functions and substitute the resulting expression for $\tilde{\omega}(\mathbf{r}, t)$ back into Eq. (317). Implementing a closure relation of the form (313), a kinetic equation can be obtained for $\bar{\omega}$. In the single level approximation, it reads [34]:

$$
\begin{aligned}
& \frac{\partial \bar{\omega}}{\partial t}+\overline{\mathbf{u}} \nabla \bar{\omega}=\epsilon^{2} \frac{\partial}{\partial r^{\mu}} \int_{0}^{t} d s \int d^{2} \mathbf{r}^{\prime} V^{\mu}\left(\mathbf{r}^{\prime} \rightarrow \mathbf{r}\right)_{t} \\
& \times\left\{V^{\nu}\left(\mathbf{r}^{\prime} \rightarrow \mathbf{r}\right) \bar{\omega}^{\prime}\left(\sigma_{0}-\bar{\omega}^{\prime}\right) \frac{\partial \bar{\omega}}{\partial r^{\nu}}+V^{\nu}\left(\mathbf{r} \rightarrow \mathbf{r}^{\prime}\right) \bar{\omega}\left(\sigma_{0}-\bar{\omega}\right) \frac{\partial \bar{\omega}^{\prime}}{\partial r^{\prime \nu}}\right\}_{t-s}
\end{aligned}
$$

Assuming in addition that the decorrelation time $\tau$ is short, as in the stochastic model of Robert \& Rosier [110], we are led to the following equation

$$
\begin{gathered}
\frac{\partial \bar{\omega}}{\partial t}+\overline{\mathbf{u}} \nabla \bar{\omega}=\frac{\epsilon^{2} \tau}{8 \pi^{2}} \frac{\partial}{\partial r^{\mu}} \int d^{2} \mathbf{r}^{\prime} K^{\mu \nu}\left(\mathbf{r}^{\prime}-\mathbf{r}\right)\left\{\bar{\omega}^{\prime}\left(\sigma_{0}-\bar{\omega}^{\prime}\right) \frac{\partial \bar{\omega}}{\partial r^{\nu}}-\bar{\omega}\left(\sigma_{0}-\bar{\omega}\right) \frac{\partial \bar{\omega}^{\prime}}{\partial r^{\prime \nu}}\right\}, \\
K^{\mu \nu}\left(\mathbf{r}^{\prime}-\mathbf{r}\right)=\frac{\xi^{2} \delta^{\mu \nu}-\xi^{\mu} \xi^{\nu}}{\xi^{4}},
\end{gathered}
$$

with $\boldsymbol{\xi}=\mathbf{r}^{\prime}-\mathbf{r}$. This equation includes a diffusion and a drift, as in the MEPP, but these terms are obtained here directly from a local average of the Euler equation. On the other hand, the conservation of angular momentum results from the symmetry of the diffusion current instead of an ad hoc Lagrange multiplier. This symmetric structure respects in addition the invariance properties of the Euler equation. Finally, a $H$-theorem for the Fermi-Dirac entropy

$$
S=-\int\left\{\frac{\bar{\omega}}{\sigma_{0}} \ln \frac{\bar{\omega}}{\sigma_{0}}+\left(1-\frac{\bar{\omega}}{\sigma_{0}}\right) \ln \left(1-\frac{\bar{\omega}}{\sigma_{0}}\right)\right\} d^{2} \mathbf{r}
$$

can be derived from this kinetic equation instead of being postulated as in the MEPP. Our approach provides therefore an alternative, dynamical, justification of the mixing entropy introduced by Miller [95] and Robert \& Sommeria [112] at statistical equilibrium.

Equations (320) and (321) are similar to the kinetic equations (238) and (239) obtained for point vortices. There are, however, two important differences: (i) the drift and the diffusion involve the product $\bar{\omega} \times\left(\sigma_{0}-\bar{\omega}\right)$ instead of $\langle\omega\rangle$. This nonlinearity ensures that the constraint $\bar{\omega} \leq \sigma_{0}$ is satisfied at any time. (ii) The diffusion coefficient is proportional to the circulation $\sigma_{0} \epsilon^{2}$ of a completely filled 
macrocell, instead of the circulation $\gamma$ of a point vortex. In general, $\sigma_{0} \epsilon^{2} \gg \gamma$ so that the relaxation by collisionless mixing is much more rapid than the collisional relaxation. From the above theory, we find that the time scale of the violent relaxation is of order $t_{D}$, the dynamical time, whereas the collisional relaxation of point vortices operates on a time scale $\sim(N / \ln N) t_{D}$.

If we are close to equilibrium, we can implement a "thermal bath approximation" and replace the vorticity $\omega^{\prime} \equiv \omega\left(\mathbf{r}^{\prime}, t\right)$ by its equilibrium form (292) 34. Then, Eq. (320) reduces to the equation (307) derived from the MEPP and the diffusion coefficient coincides with the estimate (316) based on the passive scalar model $\left(\omega_{2}=\bar{\omega}\left(\sigma_{0}-\bar{\omega}\right)\right.$ in the single level approximation). All these results are satisfactory. However, Eq. (320) does not conserve energy and this marks a flaw in the previous description. It is probable that this constraint is broken by the small correlation time hypothesis, but we do not see how to simplify Eq. (319) further without this assumption. We could try to satisfy the energy constraint by introducing a $\delta$-function term in Eq. (320) as in Eq. (245) but it is not clear how one can justify this procedure in the present context. The conservation of energy (which is intimately related to the form of the drift term) is the most serious problem that we have encountered in trying to develop a kinetic theory of 2D turbulence. Up to date, it has not been answered satisfactory. Further scrutinity should be given to the non markovian kinetic equation (319) which may conserve energy although we were not able to prove it.

It has to be emphasized that the quasilinear theory is not a theory of violent relaxation in the usual sense since it only applies to the late quiescent stages of the relaxation ("gentle relaxation"), when the fluctuations have weaken and a linearization procedure can be implemented. In order to give a more relevant description of violent relaxation, it will be important in future works to make a link between statistical mechanics and chaotic dynamics, in particular in the point vortex model. This may give a new estimate of the diffusion coefficient and of the decorrelation time $\tau$, which in this context could be related to a Lyapunov exponent. We feel that this track is an important one to make progress in the understanding of $2 \mathrm{D}$ turbulence and point vortex dynamics.

In the quasilinear theory, it is implicitly assumed that the decomposition $\omega=\bar{\omega}+\tilde{\omega}$ is obvious and that $\bar{\omega}$ should be regarded as a statistical average of $\omega(\mathbf{r}, t, \zeta)$ over different realizations $\zeta$ of the flow. This implies in particular that $\overline{\bar{\omega}}=\bar{\omega}$. However, a different approach is considered by Laval et al. [87] and Bouchet 12] who define $\bar{\omega}$ as the convolution of $\omega(\mathbf{r}, t)$ with a Gaussian window of size $\epsilon$ (or by a truncation in Fourier space). In that case $\overline{\bar{\omega}} \neq \bar{\omega}$ and new terms arise in Eq. (317), in particular a term $\mathbf{J}_{d}=\bar{\omega} \overline{\mathbf{u}}-\bar{\omega} \overline{\mathbf{u}}$ which dominates over the others [12]. When $\epsilon \rightarrow 0$, this term becomes equivalent to an anisotropic diffusion $\epsilon^{2} \Sigma^{\prime} \nabla \bar{\omega}$ with a diffusion coefficient (or turbulent viscosity) related to the stress tensor $\Sigma_{i j}^{\prime}=\partial_{i} \bar{u}_{j}$. This term alone conserves energy but there exist directions in which the viscosity is negative leading to instabilities. In order to circumvent this difficulty, Bouchet [12] proposes to project the diffusion current on directions in which the viscosity is positive and to introduce a drift term, as in the MEPP, in order to recover the conservation of energy lost by this procedure. 
This leads to an operational subgridscale model of 2D turbulence which appears to be more efficient than other parametrizations. As for the MEPP, its drawback is its ad hoc nature but it is not clear at present if it will be possible one day to do much better and derive a parametrization of $2 \mathrm{D}$ turbulence from first principles as attempted in the quasilinear theory.

\subsection{The limits $t \rightarrow+\infty$ and $N \rightarrow+\infty$}

We have indicated previously that a system of point vortices or point mass stars can achieve two successive equilibrium states. On a short time scale, the correlations between particles have not yet developed and the system is described by the Vlasov (or Euler) equation. In this regime, the dynamics is collisionless. Yet, for systems with long-range interactions, the collective nature of the evolution is responsible for an effective relaxation process, called violent relaxation, which leads to a metaequilibrium state (292) (293) on a very short time scale. On a longer time scale, the fluctuations of the potential have died away and the developement of correlations between stars or between point vortices leads to another, slower, relaxation process. This corresponds to the "collisional" regime. This second process is more standard and leads to a true equilibrium state (44)(101). In the case of continuous vorticity fields (instead of point vortices), the second stage is replaced by a viscous decay of the vorticity due to inherent viscosity.

We can discuss these two successive equilibrium states in a slightly different manner. Let us consider a fixed interval of time and let the number of particles $N \rightarrow+\infty$. In that case, the system is rigorously described by the Vlasov (or Euler) equation, and a metaequilibrium state is achieved on a timescale independant on $N$. Alternatively, if we fix $N$ and let $t \rightarrow+\infty$, the system will relax to the true equilibrium state resulting from a collisional evolution. These two equilibrium states are of course physically distinct. This implies that the order of the limits $N \rightarrow+\infty$ and $t \rightarrow+\infty$ is not interchangeable.

In the process of violent relaxation, the statistical mechanics is not as firmly established as in the collisional regime, although it is often the process of most interest. Indeed, the mixing required for the validity of the ergodic hypothesis is fed by the fluctuations of the potential. As these fluctuations decay as we approach equilibrium (by definition!), the mixing becomes less and less efficient and this can lead to an incomplete relaxation. Since the Boltzmann-Gibbs entropy does not always give a good description of the equilibrium state, it has been proposed sometimes to use a wider class of functionals to describe the process of violent relaxation 122 . Among them, the $q$-entropies introduced by Tsallis 123. have been shown to give in some cases a good fit of the equilibrium state. However, since the value of $q$ needs to be adjusted in each case, it is not clear whether this agreement is the signal of a generalized thermodynamics or just a coincidence 2 . It is clear that the Boltzmann entropy does not always give a

\footnotetext{
${ }^{2}$ In 2D turbulence, Boghosian [10 justifies a form of minimum enstrophy principle from Tsallis thermodynamics in order to interpret the experimenal results of Huang \& Driscoll 66] in a magnetized plasma. This is because the enstrophy $\Gamma_{2}=\int \omega^{2} d^{2} \mathbf{r}$
} 
perfect description of the equilibrium state but there is no convincing reason, up to date, why the system would select another "universal" form of entropy in the context of violent relaxation [44. In any case, the formalism developed by Tsallis and co-workers is nice to generalize to a wider class of functionals the results obtained with the Boltzmann entropy. Since this generalization is often analytically tractable (as it leads to power laws), this may explain the interest and the attractive nature of this approach.

\section{Conclusion}

The statistical mechanics of $2 \mathrm{D}$ vortices and stellar systems appear to be remarkably similar despite the different nature of these systems. We have tried to develop this analogy in different directions. First of all, the $N$-star and $N$ vortex problems both involve an unshielded long-range potential generated by the density of particles themselves. At statistical equilibrium, these systems are described by a Boltzmann-Poisson equation whose solutions characterize organized states (at negative temperatures for vortices). In the case of stars, the relaxation towards equilibrium can be viewed as a competition between a diffusion and a friction. We have proposed to describe the relaxation of point vortices similarly in terms of a diffusion and a drift. The diffusion is due to the fluctuations of the force experienced by a star or to the fluctuations of the velocity field moving a vortex. The statistics of these fluctuations can be studied by similar mathematical methods. The fluctuations of the gravitational field are described by a particular Lévy law, called the Holtzmark distribution, and the fluctuations of the velocity of vortices are described by a marginal Gaussian distribution, intermediate between Gaussian and Lévy laws. The friction experienced by a star is due fundamentally to the inhomogeneity of the velocity distribution of the stellar cloud. Analogously, the drift experienced by a vortex results from the spatial inhomogeneity of the vortex cloud. The friction and the drift can be understood similarly in terms of a polarization process and a back reaction of the system. In the thermal bath approximation, the coefficients of friction and drift are given by an Einstein relation and the one-body distribution function satisfies a Fokker-Planck equation. Further away from equilibrium, the collisional dynamics of stars is described by the gravitational Landau equation. We have derived a new kinetic equation that should be appropriate to the "collisional" relaxation of point vortices.

A system of stars or vortices can also undergo a form of violent relaxation. This is essentially a collisionless process driven by the rapid fluctuations of the potential as a result of collective effects (chaotic mixing). In this collisionless

is a particular $q$-entropy. However, this is essentially coincidental and the minimum enstrophy principle can lead to inconsistencies as discussed in [16]. In astrophysics, Tsallis entropies lead to pure polytropes [106]. These distribution functions are known for a long time but they do not give a particularly good description of elliptical galaxies or other stellar systems. Therefore, the relevance of Tsallis generalized thermodynamics in 2D turbulence and stellar dynamics remains questionable 16.39. 
regime, the stars and the vortices are described by the Vlasov-Poisson and the Euler-Poisson systems. These equations present a similar structure and a statistical mechanics can be developed to predict the "most probable state" resulting from a complex evolution driven by a mixing process. The relaxation towards equilibrium can be incomplete and an out-of-equilibrium study is necessary to understand what limits relaxation and causes a kinetic confinement of the system in a "maximum entropy bubble". Relaxation equations have been derived either from a heuristic Maximum Entropy Production Principle or from a more controllable kinetic theory, in an asymptotic regime of the dynamics (gentle relaxation) in which a quasilinear approximation can be implemented.

It should be noted that many results presented in this paper, in particular those corresponding to the kinetic theory of $2 \mathrm{D}$ vortices developed in Secs. 3.5. are very recent and need to be completed and further discussed. In particular, it appears indispensable to carry out extensive numerical simulations to test their relevance and determine their domains of applicability. It is plausible that the true dynamics of stars and vortices is more complex than the picture that has been given here. In addition, the description of chaos in these systems has not been addressed at all in this paper although it is presumably an essential ingredient to understand their dynamics. We are thus far from reaching a complete understanding of these systems with long-range interactions. We feel, however, that the analogy between the statistical mechanics of stars and vortices that we have investigated is correct in its mains lines and should lead again to fruitful developements.

\section{Appendix A: The calculation of the diffusion coefficient}

In this Appendix, we calculate the diffusion coefficient of a point vortex evolving in an inhomogeneous vortex cloud, using the Kubo formula. To evaluate the velocity autocorrelation function $C(\tau)=\langle V(t) V(t-\tau)\rangle$, we shall assume that between $t$ and $t-\tau$, the point vortices follow the streamlines of the equilibrium flow. This is a reasonable approximation in the case of strong shears.

\subsection{Unidirectional flow}

The trajectory of a point vortex advected by a unidirectional equilibrium flow is simply:

$$
\begin{gathered}
y(t-\tau)=y(t), \\
x(t-\tau)=x(t)-\langle V\rangle_{e q}(y) \tau .
\end{gathered}
$$

The velocity auto-correlation function appearing in Eq. (185) can be written explicitly

$$
\begin{gathered}
C(\tau)=\frac{N \gamma^{2}}{4 \pi^{2}} \int d x_{1} d y_{1} \frac{x_{1}-x}{\left(x_{1}-x\right)^{2}+\left(y_{1}-y\right)^{2}}(t) \\
\times \frac{x_{1}-x}{\left(x_{1}-x\right)^{2}+\left(y_{1}-y\right)^{2}}(t-\tau) P_{e q}(y),
\end{gathered}
$$


where we have used Eq. (14). The second term involves the quantity

$$
\left(x_{1}-x\right)(t-\tau)=x_{1}-x+\left(\langle V\rangle_{e q}\left(y_{1}\right)-\langle V\rangle_{e q}(y)\right) \tau .
$$

Since the integral in Eq. (325) diverges as $\mathbf{r}_{1} \rightarrow \mathbf{r}$, we can make a local approximation and expand the velocity difference in a Taylor series in $y_{1}-y$. To first order, we have

$$
\langle V\rangle_{e q}\left(y_{1}\right)-\langle V\rangle_{e q}(y) \simeq-\Sigma(y)\left(y_{1}-y\right)
$$

where $\Sigma(y)$ is the local shear of the flow. Introducing the variables $X \equiv x_{1}-x$ and $Y \equiv y_{1}-y$, we obtain

$$
C(\tau)=\frac{N \gamma^{2}}{4 \pi^{2}} P_{e q}(y) \int d X d Y \frac{X}{X^{2}+Y^{2}} \frac{X+\Sigma(y) Y \tau}{(X+\Sigma(y) Y \tau)^{2}+Y^{2}} .
$$

The integration over $X$ can be performed easily since the integrand is just a rational function of polynomials. After straightforward calculations, we find

$$
C(\tau)=\frac{N \gamma^{2}}{4 \pi} P_{e q}(y) \frac{1}{1+\frac{1}{4} \Sigma^{2}(y) \tau^{2}} \int_{0}^{+\infty} \frac{d Y}{Y} .
$$

The integral over $Y$ diverges logarithmically for both small and large $Y$. The reason for this divergence has been explained in Sec. 3. Introducing two cut-offs at scales $d$ (the inter-vortex distance) and $R$ (the domain size) and noting that $\ln (R / d) \sim \frac{1}{2} \ln N$, we obtain

$$
C(\tau)=\frac{N \gamma^{2}}{8 \pi} \ln N \frac{1}{1+\frac{1}{4} \Sigma^{2}(y) \tau^{2}} P_{e q}(y) .
$$

For $\tau \rightarrow+\infty$, the correlation function decreases like $\tau^{-2}$. This is a slow decay but it is sufficient to ensure the convergence of the diffusion coefficient (185). Using

$$
\int_{0}^{t} C(\tau) d \tau=\frac{N \gamma^{2}}{4 \pi} \frac{\ln N}{|\Sigma(y)|} \arctan \left(\frac{1}{2}|\Sigma(y)| t\right) P_{e q}(y),
$$

and taking the limit $t \rightarrow+\infty$, we establish Eq. (189).

\subsection{Axisymmetric flow}

In an axisymmetric flow, the trajectory of a point vortex takes the simple form:

$$
\begin{gathered}
r(t-\tau)=r(t), \\
\theta(t-\tau)=\theta(t)-\frac{\langle V\rangle_{e q}(r)}{r} \tau .
\end{gathered}
$$


As indicated in Sec. 4.2, we are particularly interested by the $r(t) r(t-\tau)$ component of the correlation tensor. Let us introduce the separation $\delta \mathbf{r} \equiv \mathbf{r}_{1}-\mathbf{r}$ between the field vortex 1 and the test vortex. In the local approximation, $\delta \mathbf{r}$ can be considered as a small quantity. Therefore, we can write

$$
\begin{gathered}
\delta \mathbf{r}=r \delta \theta \mathbf{e}_{\theta}+\delta r \mathbf{e}_{r} \equiv X \mathbf{e}_{\theta}+Y \mathbf{e}_{r}, \\
d^{2} \mathbf{r}_{1}=d^{2}(\delta \mathbf{r})=d X d Y .
\end{gathered}
$$

With these notations, the correlation function appearing in Eq. (188) can be rewritten

$$
C(\tau)=\frac{N \gamma^{2}}{4 \pi^{2}} P_{e q}(r) \int d X d Y \frac{X}{X^{2}+Y^{2}}(t) \frac{X}{X^{2}+Y^{2}}(t-\tau) .
$$

Now,

$$
\begin{aligned}
& Y(t-\tau)=\delta r(t-\tau)=r_{1}(t-\tau)-r(t-\tau) \\
& =r_{1}(t)-r(t)=Y(t)=Y
\end{aligned}
$$

and

$$
\begin{aligned}
& X(t-\tau)=r(t-\tau) \delta \theta(t-\tau)=r(t-\tau)\left(\theta_{1}(t-\tau)-\theta(t-\tau)\right) \\
& =r\left(\theta_{1}(t)-\theta(t)-\left(\frac{\langle V\rangle_{e q}\left(r_{1}\right)}{r_{1}}-\frac{\langle V\rangle_{e q}(r)}{r}\right) \tau\right) .
\end{aligned}
$$

In the local approximation, we can expand the last term in Eq. (338) in a Taylor series in $r_{1}-r$. This yields

$$
X(t-\tau)=r\left(\theta_{1}(t)-\theta(t)\right)-r \frac{d}{d r}\left(\frac{\langle V\rangle_{e q}(r)}{r}\right)\left(r_{1}-r\right) \tau=X-\Sigma(r) Y \tau,
$$

where $\Sigma(r)$ is the local shear of the flow. Substituting Eqs. (337) and (339) in Eq. (336), we get

$$
C(\tau)=\frac{N \gamma^{2}}{4 \pi^{2}} P_{e q}(r) \int d X d Y \frac{X}{X^{2}+Y^{2}} \frac{X-\Sigma(r) Y \tau}{(X-\Sigma(r) Y \tau)^{2}+Y^{2}} .
$$

This integral is similar to Eq. (328), so we again obtain Eq. (189).

\section{Appendix B: Calculation of the memory function}

In this Appendix, we calculate the memory function that occurs in Eq. 236). If we assume that $P=P(r, t)$, then Eq. (236) simplifies to

$$
\frac{\partial P}{\partial t}=-\frac{1}{r} \frac{\partial}{\partial r}\left(r J_{r}\right)
$$


where

$$
\begin{aligned}
& J_{r}=-N \int_{0}^{t} d \tau \int d^{2} \mathbf{r}_{1} V_{r(t)}(1 \rightarrow 0)_{t} \\
& \times\left\{V_{r(t-\tau)}(1 \rightarrow 0) P_{1} \frac{\partial P}{\partial r}-V_{r_{1}(t-\tau)}(1 \rightarrow 0) P \frac{\partial P_{1}}{\partial r_{1}}\right\}_{t-\tau},
\end{aligned}
$$

and where $V_{r(t)}(1 \rightarrow 0)$ is the component of the vector $\mathbf{V}(1 \rightarrow 0)$ in the direction of $\mathbf{r}(t)$. If we denote by $(r(t), \theta(t))$ and $\left(r_{1}(t), \theta_{1}(t)\right)$ the polar coordinates that specify the position of the point vortices 0 and 1 at time $t$, we easily find that

$$
V_{r(t)}(1 \rightarrow 0)=-\frac{\gamma}{2 \pi} \frac{r_{1} \sin \left(\theta-\theta_{1}\right)}{r_{1}^{2}+r^{2}-2 r r_{1} \cos \left(\theta-\theta_{1}\right)}
$$

We shall assume that between $t$ and $t-\tau$, the point vortices follow circular trajectories with angular velocity $\Omega(r, t)$. In that case, $r(t-\tau)=r$ and $\theta(t-\tau)=$ $\theta-\Omega(r, t) \tau$. Then, we obtain

$$
V_{r(t-\tau)}(1 \rightarrow 0)=-\frac{\gamma}{2 \pi} \frac{r_{1} \sin \left(\theta-\theta_{1}-\Delta \Omega \tau\right)}{r_{1}^{2}+r^{2}-2 r r_{1} \cos \left(\theta-\theta_{1}-\Delta \Omega \tau\right)}
$$

with

$$
\Delta \Omega=\Omega(r, t)-\Omega\left(r_{1}, t\right)
$$

We find similarly that $V_{r_{1}(t-\tau)}(1 \rightarrow 0)=\frac{r}{r_{1}} V_{r(t-\tau)}(1 \rightarrow 0)$. Our previous assumptions also imply that $P(r(t-\tau), t-\tau) \simeq P(r, t)$ between $t$ and $t-\tau$. In words, this means that the correlation time is smaller than the time scale on which the average vorticity changes appreciably. We do not assume that it is much smaller as in Sec. 5.5, so this approximation is not over restrictive. In that case, the diffusion current becomes

$$
\begin{aligned}
& J_{r}=-N \int_{0}^{+\infty} d \tau \int_{0}^{2 \pi} d \theta_{1} \int_{0}^{+\infty} r r_{1} d r_{1} V_{r(t)}(1 \rightarrow 0) \\
& \times V_{r(t-\tau)}(1 \rightarrow 0)\left[\frac{1}{r} P_{1} \frac{\partial P}{\partial r}-\frac{1}{r_{1}} P \frac{\partial P_{1}}{\partial r_{1}}\right]
\end{aligned}
$$

where the time integral has been extended to $+\infty$. We now need to evaluate the memory function

$$
M=\int_{0}^{+\infty} d \tau \int_{0}^{2 \pi} d \theta_{1} V_{r(t)}(1 \rightarrow 0) V_{r(t-\tau)}(1 \rightarrow 0) .
$$

Introducing the notations $\phi=\theta_{1}-\theta$ and

$$
\lambda=\frac{2 r r_{1}}{r_{1}^{2}+r^{2}}<1
$$


we have explicitly

$$
M=\left(\frac{\gamma \lambda}{4 \pi r}\right)^{2} \int_{0}^{+\infty} d \tau \int_{0}^{2 \pi} d \phi \frac{\sin \phi}{1-\lambda \cos \phi} \frac{\sin (\phi+\Delta \Omega \tau)}{1-\lambda \cos (\phi+\Delta \Omega \tau)} .
$$

This can also be written

$$
M=\left(\frac{\gamma}{4 \pi r}\right)^{2} \int_{0}^{+\infty} d \tau \int_{0}^{2 \pi} d \phi V^{\prime}(\phi) V^{\prime}(\phi+\Delta \Omega \tau),
$$

where

$$
V(\phi)=\ln (1-\lambda \cos \phi) .
$$

We now write the function $V(\phi)$ in the form of a Fourier series,

$$
V(\phi)=\sum_{n=-\infty}^{+\infty} a_{n} e^{i n \phi} \quad \text { with } \quad a_{n}=\frac{1}{2 \pi} \int_{-\pi}^{\pi} V(\phi) e^{-i n \phi} d \phi .
$$

The memory function becomes

$$
M=-\frac{1}{2}\left(\frac{\gamma}{4 \pi r}\right)^{2} \int_{-\infty}^{+\infty} d \tau \int_{0}^{2 \pi} d \phi \sum_{n, m=-\infty}^{+\infty} n m a_{n} a_{m} e^{i(n+m) \phi} e^{i m \Delta \Omega \tau}
$$

Carrying out the integrations on $\phi$ and $\tau$ using the integral representation of the delta function

$$
\delta(x)=\frac{1}{2 \pi} \int_{-\infty}^{+\infty} e^{-i \rho x} d \rho
$$

we are left with

$$
M=-\frac{\gamma^{2}}{8 r^{2}} \sum_{n, m=-\infty}^{+\infty} n m a_{n} a_{m} \delta_{n,-m} \delta(m \Delta \Omega)=\frac{\gamma^{2}}{8 r^{2}} \delta(\Delta \Omega) \sum_{n=-\infty}^{+\infty}|n| a_{n}^{2} .
$$

It remains for us to evaluate the series that appears in the last expression of the memory function. Using the identities

$$
\begin{gathered}
\int_{0}^{\pi} \ln (1-\lambda \cos \phi) \cos (n \phi) d \phi=-\frac{\pi}{n}\left(\frac{1}{\lambda}-\sqrt{\frac{1}{\lambda^{2}}-1}\right)^{n} \quad(n>0), \\
\int_{0}^{\pi} \ln (1-\lambda \cos \phi) d \phi=\pi \ln \left(\frac{1}{2}+\frac{\sqrt{1-\lambda^{2}}}{2}\right)
\end{gathered}
$$

and the definition (348) of $\lambda$, we find that $a_{0}<\infty$ and, for $n>0$,

$$
a_{n}=-\frac{1}{n}\left(\frac{\left(r_{1}^{2}+r^{2}\right)-\left|r_{1}^{2}-r^{2}\right|}{2 r r_{1}}\right)^{n}=-\frac{1}{n}\left(\frac{r_{<}}{r_{>}}\right)^{n},
$$


where $r_{>}$(resp. $r_{<}$) is the biggest (resp. smallest) of $r$ and $r_{1}$. Therefore, the value of the series is

$$
\sum_{n=-\infty}^{+\infty}|n| a_{n}^{2}=2 \sum_{n=1}^{+\infty} n a_{n}^{2}=2 \sum_{n=1}^{+\infty} \frac{1}{n}\left(\frac{r_{<}}{r_{>}}\right)^{2 n}=-2 \ln \left[1-\left(\frac{r_{<}}{r_{>}}\right)^{2}\right] .
$$

The memory function takes the form

$$
M=-\frac{\gamma^{2}}{4 r^{2}} \delta(\Delta \Omega) \ln \left[1-\left(\frac{r_{<}}{r_{>}}\right)^{2}\right]
$$

and the diffusion current in the axisymmetric case can be written

$$
J_{r}=\frac{N \gamma^{2}}{4 r} \int_{0}^{+\infty} r_{1} d r_{1} \delta\left(\Omega-\Omega_{1}\right) \ln \left[1-\left(\frac{r_{<}}{r_{>}}\right)^{2}\right]\left\{\frac{1}{r} P_{1} \frac{\partial P}{\partial r}-\frac{1}{r_{1}} P \frac{\partial P_{1}}{\partial r_{1}}\right\}
$$

This leads to the kinetic equation (243).

\section{References}

1. T. Agekyan, Sov. Astron. 5, 809 (1962).

2. V.A. Antonov, Vest. Leningr. Gos. Univ. 7, 135 (1962).

3. E.B. Aronson and C.J. Hansen, "Thermal equilibrium states of a classical system with gravitation", Astrophys. J. 177, 145 (1972).

4. R. Balescu, Statistical Mechanics of Charged Particles (Interscience, New York, 1963).

5. P. Barge and J. Sommeria, "Did planet formation begin inside persistent gaseous vortices?", Astron. Astrophys. 295, L1 (1995).

6. J. Barré, D. Mukamel and S. Ruffo, "Inequivalence of ensembles in a system with long-range interactions", Phys. Rev. Lett. 87, 030601 (2001).

7. P. Bertrand, Contribution à l'étude de modèles mathématiques de plasmas non collisionnels, PhD thesis, Université de Nancy I (1972).

8. N. Bilic and R.D. Viollier, "Gravitational phase transition of heavy neutrino matter", Phys. Lett. B 408, 75 (1997).

9. J. Binney and S. Tremaine, Galactic Dynamics (Princeton Series in Astrophysics, 1987).

10. B.M. Boghosian, "Thermodynamic description of the relaxation of $2 \mathrm{D}$ turbulence using Tsallis statistics" Phys. Rev. E 53, 4754 (1996).

11. J.P. Bouchaud and A. Georges, "Anomalous diffusion in disordered media: statistical mechanisms, models and physical applications", Phys. Rep. 195, 127 (1990).

12. F. Bouchet, Mécanique statistique pour des écoulements géophysiques, $\mathrm{PhD}$ thesis, Université J. Fourier (2001).

13. F. Bouchet, P.H. Chavanis and J. Sommeria, "Statistical mechanics of Jupiter's Great Red Spot in the shallow water model", preprint.

14. F. Bouchet and J. Sommeria, "Emergence of intense jets and Jupiter Great Red Spot as maximum entropy structures", J. Fluid. Mech. 464, 165 (2002). 
15. A. Bracco, P.H. Chavanis, A. Provenzale and E. Spiegel, "Particle aggregation in a turbulent Keplerian flow", Phys. Fluids 11, 2280 (1999).

16. H. Brands, P.H. Chavanis, R. Pasmanter and J. Sommeria, "Maximum entropy versus minimum enstrophy vortices", Phys. Fluids 11, 3465 (1999).

17. E. Caglioti, P.L. Lions, C. Marchioro and M. Pulvirenti, "A special class of stationary flows for two-dimensional Euler equations: a statistical mechanics description", Commun. Math. Phys. 143, 501 (1992).

18. G.F. Carnevale, J.C. McWilliams, Y. Pomeau, J.B. Weiss and W.R. Young, "Evolution of vortex statistics in two-dimensional turbulence", Phys. Rev. Lett. 66, 2735 (1991).

19. S. Chandrasekhar, An Introduction to the Theory of Stellar Structure (Dover 1939).

20. S. Chandrasekhar, "A statistical theory of stellar encounters", Astrophys. J. 94, 511 (1941).

21. S. Chandrasekhar, Principles of stellar dynamics (Dover 1942).

22. S. Chandrasekhar, "Stochastic problems in physics and astronomy", Rev. Mod. Phys. 15, 1 (1943).

23. S. Chandrasekhar, "Dynamical friction. I. General considerations: the coefficient of dynamical friction", Astrophys. J. 97, 255 (1943).

24. S. Chandrasekhar, "The statistics of the gravitational field arising from a random distribution of stars: III. The correlations in the forces acting at two points separated by a finite distance", Astrophys. J. 99, 25 (1944).

25. S. Chandrasekhar, "The statistics of the gravitational field arising from a random distribution of stars: IV. The stochastic variation of the force acting on a star", Astrophys. J. 99, 47 (1944).

26. S. Chandrasekhar, "Brownian motion, dynamical friction and stellar dynamics", Rev. Mod. Phys. 21, 383 (1949).

27. S. Chandrasekhar \& J. von Neumann, "The statistics of the gravitational field arising from a random distribution of stars I. The speed of fluctuations", Astrophys. J. 95, 489 (1942).

28. S. Chandrasekhar \& J. von Neumann "The statistics of the gravitational field arising from a random distribution of stars II. The speed of fluctuations; dynamical friction; spatial correlations", Astrophys. J. 97, 1 (1943).

29. P.H. Chavanis, Contribution à la mécanique statistique des tourbillons bidimensionnels. Analogie avec la relaxation violente des systèmes stellaires, Thèse de doctorat, Ecole Normale Supérieure de Lyon (1996).

30. P.H. Chavanis, "Systematic drift experienced by a point vortex in two-dimensional turbulence", Phys. Rev. E 58, R1199 (1998).

31. P.H. Chavanis, "On the coarse-grained evolution of collisionless stellar systems", Mon. Not. R. astr. Soc. 300, 981 (1998).

32. P.H. Chavanis, "From Jupiter's Great Red Spot to the structure of galaxies: statistical mechanics of two-dimensional vortices and stellar systems", Annals of the New York Academy of Sciences 867, 120 (1998).

33. P.H. Chavanis, "Trapping of dust by coherent vortices in the solar nebula", Astron. Astrophys. 356, 1089 (2000).

34. P.H. Chavanis, "Quasilinear theory of the 2D Euler equation", Phys. Rev. Lett. 84, 5512 (2000).

35. P.H. Chavanis, "On the analogy between two-dimensional vortices and stellar systems", Proceedings of the IUTAM Symposium on Geometry and Statistics of Turbulence (2001), T. Kambe, T. Nakano and T. Miyauchi Eds. (Kluwer Academic Publishers). 
36. P.H. Chavanis, "Kinetic theory of point vortices: diffusion coefficient and systematic drift", Phys. Rev. E 64, 026309 (2001).

37. P.H. Chavanis, "Gravitational instability of finite isothermal spheres", Astron. Astrophys. 381, 340 (2002).

38. P.H. Chavanis, "Gravitational instability of finite isothermal spheres in general relativity. Analogy with neutron stars", Astron. Astrophys. 381, 709 (2002).

39. P.H. Chavanis, "Gravitational instability of polytropic spheres and generalized thermodynamics", Astron. Astrophys. 386, 732 (2002).

40. P.H. Chavanis, "Effective velocity created by a point vortex in two-dimensional hydrodynamics", Phys. Rev. E 65, 056302 (2002).

41. P.H. Chavanis, "Phase transitions in self-gravitating systems. Self-gravitating fermions and hard sphere models", Phys. Rev. E 65, 056123 (2002).

42. P.H. Chavanis, "Statistical mechanics of violent relaxation in stellar systems", Proceedings of the Conference on Multiscale Problems in Science and Technology (Springer 2002); also available on astro-ph/0212205.

43. P.H. Chavanis, "The self-gravitating Fermi gas", Proceedings of the Conference Dark2002: 4th International Heidelberg Conference on Dark Matter in Astro and Particle Physics, 4-9 Feb 2002, Cape Town, South African Astroparticles (Springer); also available on astro-ph/0205426.

44. P.H. Chavanis, "Gravitational instability of isothermal and polytropic spheres", Astron. Astrophys. in press astro-ph/0207080.

45. P.H. Chavanis and I. Ispolatov, "Phase diagram of self-attracting systems", Phys. Rev. E 66, 036109 (2002).

46. P.H. Chavanis, C. Rosier and C. Sire "Thermodynamics of self-gravitating systems", Phys. Rev. E. 66, 036105 (2002).

47. P.H. Chavanis and C. Sire, "The statistics of velocity fluctuations arising from a random distribution of point vortices: the speed of fluctuations and the diffusion coefficient", Phys. Rev. E 62, 490 (2000).

48. P.H. Chavanis and C. Sire, "The spatial correlations in the velocities arising from a random distribution of point vortices", Phys. Fluids 13, 1904 (2001).

49. P.H. Chavanis and J. Sommeria, "Classification of self-organized vortices in twodimensional turbulence: the case of a bounded domain", J. Fluid Mech. 314, 267 (1996).

50. P.H. Chavanis and J. Sommeria, "Thermodynamical approach for small-scale parametrization in 2D turbulence", Phys. Rev. Lett. 78, 3302 (1997).

51. P.H. Chavanis and J. Sommeria, "Classification of robust isolated vortices in twodimensional hydrodynamics", J. Fluid Mech. 356, 259 (1998).

52. P.H. Chavanis and J. Sommeria, "Degenerate equilibrium states of collisionless stellar systems", Mon. Not. R. astr. Soc. 296, 569 (1998).

53. P.H. Chavanis and J. Sommeria, "Statistical mechanics of the shallow water system", Phys. Rev. E 65, 026302 (2002).

54. P.H. Chavanis, J. Sommeria and R. Robert, "Statistical mechanics of twodimensional vortices and collisionless stellar systems", Astrophys. J. 471, 385 (1996).

55. K.V. Chukbar, "Statistics of two-dimensional vortices and the Holtsmark distribution," Plasma Physics Reports. 25, 77 (1999).

56. H. Cohn, "Late core collapse in star clusters and the gravothermal instability", Astrophys. J. 242, 765 (1980).

57. H.J. de Vega and N. Sanchez, "Statistical mechanics of the self-gravitating gas: I. Thermodynamical limit and phase diagrams", Nucl. Phys. B 625, 409 (2002). 
58. G.L. Eyink and H. Spohn, "Negative temperature states and large-scale, long-lived vortices in two-dimensional turbulence", J. Stat. Phys. 70, 833 (1993).

59. E. Follana and V. Laliena, "Thermodynamics of self-gravitating systems with softened potentials", Phys. Rev. E 61, 6270 (2000).

60. P. Godon and M. Livio, "The formation and role of vortices in protoplanetary disks", Astrophys. J. 537, 396 (2000).

61. A.E. Hansen, D. Marteau and P. Tabeling, "Two-dimensional turbulence and dispersion in a freely decaying system", Phys. Rev. E 58, 7261 (1998).

62. P. Hertel, W. Thirring, "Thermodynamic instability of a system of gravitating fermions" in Quanten und Felder, edited by H.P. Dürr (Vieweg, Braunschweig, 1971)

63. J. Hjorth and J. Madsen, "Statistical mechanics of galaxies", Mon. Not. R. astr. Soc. 265, 237 (1993).

64. J. Holtsmark, Ann. Phys. (Leipzig) 58, 577 (1919).

65. G. Horwitz and J. Katz, "Steepest descent technique and stellar equilibrium statistical mechanics. III. Stability of various ensembles", Astrophys. J. 222, 941 (1978).

66. X.P. Huang and C.F. Driscoll, "Relaxation of 2D turbulence to a metaequilibrium near the minimum enstrophy state", Phys. Rev. Lett. 72, 2187 (1994).

67. I.A. Ibragimov and Yu. V. Linnik, Independant and Stationary Sequences of Random Variables (Wolters-Noordhoff, Groningen, 1971).

68. S. Inagaki and D. Lynden-Bell, "Self-similar solutions for post-collapse evolution of globular clusters", Mon. Not. R. astr. Soc. 205, 913 (1983).

69. J. Jimenez, "Algebraic probability density tails in decaying isotropic twodimensional turbulence", J. Fluid Mech. 313, 223 (1996).

70. G. Joyce and D. Montgomery, "Negative temperature states for the twodimensional guiding-center plasma", J. Plasma Phys. 10, 107 (1973).

71. B.B. Kadomtsev and O.P. Pogutse, "Collisionless relaxation in systems with Coulomb interactions", Phys. Rev. Lett. 25, 1155 (1970).

72. H.E. Kandrup, "Stochastic gravitational fluctuations in a self-consistent mean field theory", Physics Reports 63, 1 (1980).

73. H.E. Kandrup, "A generalized Landau equation for a system with a self-consistent mean field: derivation from an $N$-particle Liouville equation", Astrophys. J. 244, 316 (1981).

74. H.E. Kandrup, "Dynamical friction in a mean field approximation", Astrophys. \& Space Sci. 97, 435 (1983).

75. J. Katz, "On the number of unstable modes of an equilibrium", Mon. Not. R. astr. Soc. 183, 765 (1978).

76. J. Katz and D. Lynden-Bell, "The gravothermal instability in two dimensions", Mon. Not. R. astr. Soc. 184, 709 (1978).

77. J. Katz and I. Okamoto, "Fluctuations in isothermal spheres", Mon. Not. R. astr. Soc. 317, 163 (2000).

78. E. Kazantzev, J. Sommeria and J. Verron, "Subgridscale eddy parametrization by statistical mechanics in a barotropic ocean model", J. Phys. Ocean. 28, 1017 (1998).

79. G. Kirchhoff, in Lectures in Mathematical Physics, Mechanics (Teubner, Leipzig, 1877).

80. R.H. Kraichnan, "Statistical dynamics of two-dimensional flow", J. Fluid Mech. 67, 155 (1975).

81. H.A. Kramers, Physica 7, 284 (1940).

82. B.N. Kuvshinov and T.J. Schep, "Holtsmark distribution in point vortex systems," Phys. Rev. Lett. 84, 650 (2000). 
83. G.A. Kuzmin, "Statistical mechanics of the organization into two-dimensional coherent structures", in Structural Turbulence, edited by M.A. Goldshtik (Acad. Naouk CCCP Novosibirsk, Institute of ThermoPhysics, 1982), pp. 103-114.

84. C. Lancellotti and M. Kiessling, "Self-gravitational collapse in stellar dynamics", Astrophys. J. 549, L93 (2001).

85. R.B. Larson, "A method for computing the evolution of star clusters", Mon. Not. R. astr. Soc. 147, 323 (1970).

86. J.P. Laval, P.H. Chavanis, B. Dubrulle and C. Sire, "Scaling laws and vortex profiles in 2D decaying turbulence", Phys. Rev E 63, 065301(R) (2001).

87. J.P. Laval, B. Dubrulle and S. Nazarenko, "Nonlocality of interaction of scales in the dynamics of 2D incompressible fluids", Phys. Rev. Lett. 83, 4061 (1999).

88. E.P. Lee, "Brownian motion in a stellar system", Astrophys. J. 151, 687 (1968).

89. T.S. Lundgren and Y.B. Pointin, "Statistical mechanics of two-dimensional vortices", J. Stat. Phys. 17, 323 (1977).

90. D. Lynden-Bell, "Statistical mechanics of violent relaxation in stellar systems", Mon. Not. R. astr. Soc. 136, 101 (1967).

91. D. Lynden-Bell and P.P. Eggleton, "On the consequences of the gravothermal catastrophe", Mon. Not. R. astr. Soc. 191, 483 (1980).

92. D. Lynden-Bell and R. Wood, "The gravothermal catastrophe in isothermal spheres and the onset of red-giants structure for stellar systems", Mon. Not. R. astr. Soc. 138, 495 (1968).

93. J.C. McWilliams, "The emergence of isolated coherent vortices in turbulent flow", J. Fluid Mech. 146, 21 (1984).

94. J. Michel and R. Robert, "Statistical mechanical theory of the great red spot of Jupiter", J. Phys. Stat. 77, 645 (1994).

95. J. Miller, "Statistical mechanics of the Euler equation in two dimensions", Phys. Rev. Lett. 65, 2137 (1990).

96. J. Miller, P.B. Weichman and M.C. Cross "Statistical mechanics, Euler's equation, and Jupiter's Red Spot", Phys. Rev. A 45, 2328 (1992).

97. I.A. Min, I. Mezic and A. Leonard "Lévy stable distributions for velocity and velocity difference in systems of vortex elements", Phys. Fluids 8, 1169 (1996).

98. P.K. Newton, The N-Vortex Problem: Analytical Techniques, Springer-Verlag, Applied Mathematical Sciences Vol. 145, May 2001.

99. M.V. Nezlin and E.N. Snezhkin, Rossby vortices, spiral structures, solitons (Springer-Verlag 1993).

100. E.A. Novikov "Dynamics and statistics of a system of vortices", Sov. Phys. JETP 41, 937 (1975).

101. L. Onsager, "Statistical hydrodynamics", Nuovo Cimento Suppl. 6, 279 (1949).

102. T. Padmanabhan, "Antonov instability and the gravothermal catastropherevisited", Astrophys. J. Supp. 71, 651 (1989).

103. T. Padmanabhan, "Statistical mechanics of gravitating systems", Phys. Rep. 188, 285 (1990).

104. J. Pedlosky, Geophysical fluid dynamics (Springer-Verlag, 1996).

105. M.V. Penston, "Dynamics of self-gravitating gaseous spheres-III. Analytical results in the free-fall of isothermal cases.", Mon. Not. R. astr. Soc. 144, 425 (1969).

106. A. Plastino and A.R. Plastino, "Stellar polytropes and Tsallis entropy", Phys. Lett. A 226, 257 (1997).

107. Y.B. Pointin and T.S. Lundgren, "Statistical mechanics of two-dimensional vortices in a bounded container", Phys. Fluids. 19, 1459 (1976).

108. H. Risken, The Fokker-Planck equation (Springer, 1989). 
109. R. Robert, "A maximum entropy principle for two-dimensional Euler equations", J. Stat. Phys. 65, 531 (1991).

110. R. Robert and C. Rosier, "The modelling of small scales in 2D turbulent flows: A statistical mechanical approach", J. Stat. Phys. 86, 481 (1997).

111. R. Robert and J. Sommeria, "Statistical equilibrium states for two-dimensional flows", J. Fluid Mech. 229, 291 (1991).

112. R. Robert and J. Sommeria, "Relaxation towards a statistical equilibrium state in two-dimensional perfect fluid dynamics", Phys. Rev. Lett. 69, 2776 (1992).

113. A. Salzberg and S. Prager, J. Chem. Phys. 38, 2587 (1963).

114. G. Severne and M. Luwel, "Dynamical theory of collisionless relaxation", Astrophys. \& Space Sci. 72, 293 (1980).

115. C. Sire and P.H. Chavanis, "Numerical renormalization group of vortex aggregation in 2D decaying turbulence: the role of three-body interactions", Phys. Rev. E 61, 6644 (2000).

116. C. Sire and P.H. Chavanis, "Thermodynamics and collapse of self-gravitating Brownian particles in D dimensions", Phys. Rev. E 66, 046133 (2002).

117. A.R. Smith and T.M. O'Neil "Nonaxisymmetric thermal equilibria of a cylindrically bounded guiding-center plasma or discrete vortex system", Phys. Fluids B 2, 2961 (1990).

118. J. Sommeria, C. Nore, T. Dumont and R. Robert, "Théorie statistique de la Tache Rouge de Jupiter", C.R. Acad. Sci. II 312, 999 (1991).

119. J. Sommeria, C. Staquet and R. Robert, "Final equilibrium state of a twodimensional shear layer", J. Fluid Mech. 233, 661 (1991).

120. B. Stahl, M.K.H. Kiessling and K. Schindler, "Phase transitions in gravitating systems and the formation of condensed objects", Planet. Space Sci. 43, 271 (1994).

121. P. Tanga, A. Babiano, B. Dubrulle and A. Provenzale, "Forming planetesimals in vortices", Icarus 121, 158 (1996).

122. S. Tremaine, M. Hénon and D. Lynden-Bell, "H-functions and mixing in violent relaxation", Mon. Not. R. astr. Soc. 219285 (1986).

123. C. Tsallis, "Possible generalization of Boltzmann-Gibbs statistics", J. Stat. Phys. 52479 (1988).

124. B. Turkington and N. Whitaker, "Statistical equilibrium computations of coherent structures in turbulent shear layers", SIAM J. Sci. Comput. 17, 1414 (1996).

125. J.B. Weiss and J.C. McWilliams, "Temporal scaling behavior of decaying twodimensional turbulence", Phys. Fluids A 5, 608 (1993).

126. J.B. Weiss, A. Provenzale and J.C. McWilliams, "Lagrangian dynamics in highdimensional point vortex systems", Phys. Fluids 10, 1929 (1998).

127. C.R. Willis and R.H. Picard, "Time-dependent projection-operator approach to master equations for coupled systems", Phys. Rev. A 9, 1343 (1974).

128. V.P. Youngkins and B.N. Miller, "Gravitational phase transitions in a onedimensional spherical system", Phys. Rev. E 62, 4582 (2000). 